\begin{tabular}{l}
\hline 2. To: (Receiving Organization) \\
Lockheed Mart in Hanford /77Coo \\
\hline 5. Proj./Prog./Dept./0iv.:
\end{tabular}

\section{E61945}

8. Originator Remarks:

For external release

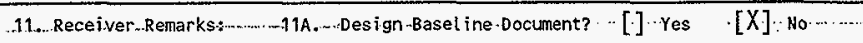

3. From: (Originating Organization)

Fluor Daniel Northwest

6. Design Authority/ Design Agent/Cog.

E. D. Johnson $/ 454$

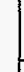

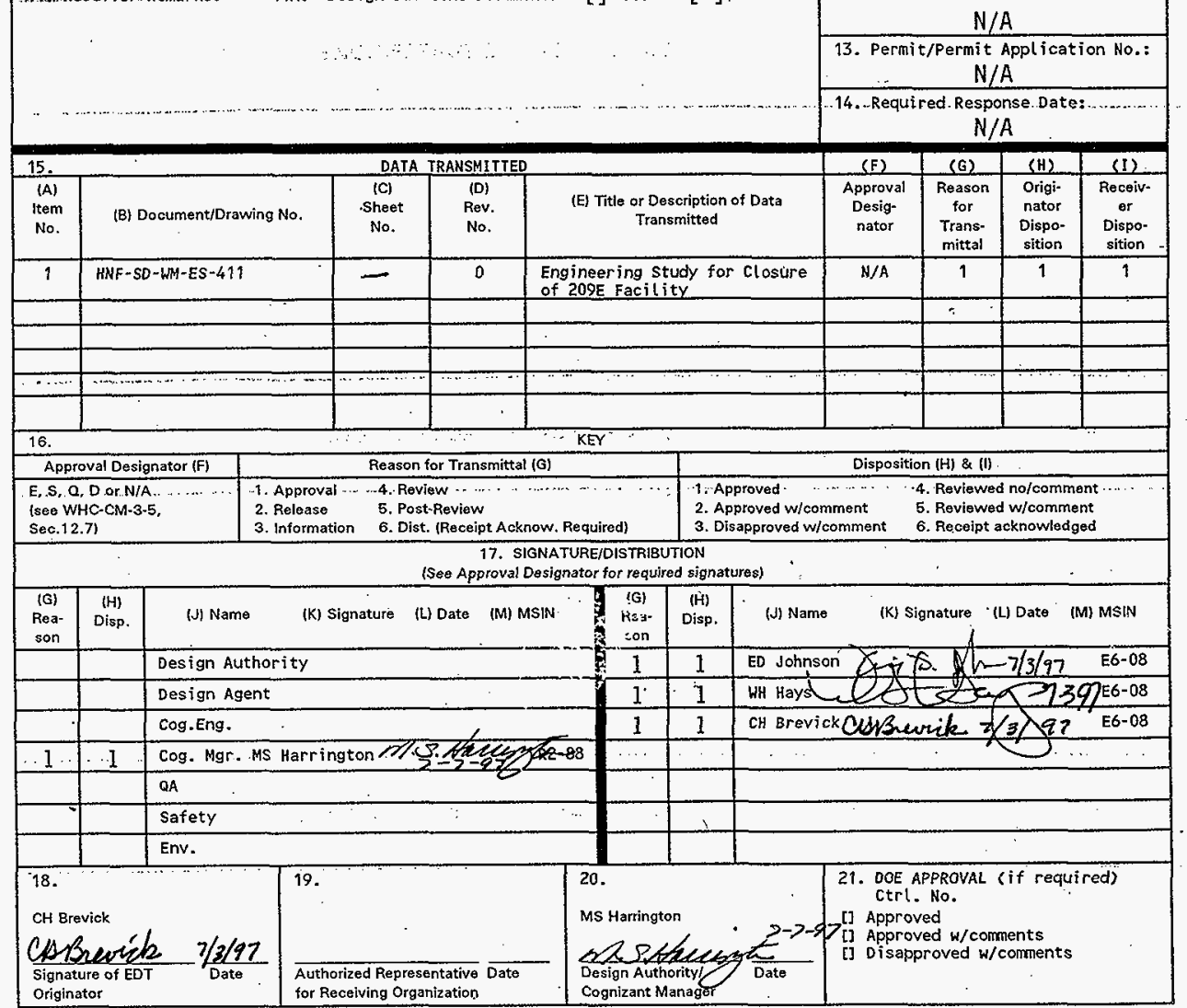

BD-7400-172-2 (05/96) GEF097 


\title{
Engineering Study For Closure of 209E Facility
}

\author{
C. H. Brevick, W. H. Hays, E. D. Johnson
}

Fluor Daniel Northwest, Ríchland, WA 99352

U.S. Department of Energy Contract DE-AC06-96RL13200

$\begin{array}{lll}\text { EDT/ECN: } & 6 / 8973 & \\ 641029-9 / 9 / 97 & \text { UC: } 2070 \\ \text { Org Code: } & 408 & \text { Charge Code: E61945 } \\ \text { B\&R Code: } & \text { EW3120074 } & \text { Total Pages: } 1 / 8\end{array}$

Key Words': Critical mass Taboratory, CML, 209E Building, non-nuclear classification, Critical Assembly Room, Mix room, criticality experiments, decontamination, decommissioning.

Abstract: This document is an engineering study for evaluating aT ternatives to determine the most cost effective closure plan for the 209E Facility, Critical Mass Laboratory. This laboratory is located in the 200 East Area of the Hanford Site and contains a Critical Assembly Room and a Mix room were criticality experiments were once performed.

TRADEMARK DISCLAIMER. Reference herein to any specific commercial product, process, or service by trade name, trademark, manufacturer, or otherwise, does not necessarily constitute or imply. its endorsement, recommendation, or favoring by the United States Government or any agency thereof or its contractors or subcontractors.

Printed in the United States of America. To obtain copies of this docurient, contact: Document Control Services, P.O. Box 950, Mailstop H6-08, Richland WA 99352, Phone (509) 372-2420; Fax (509) 376-4989.
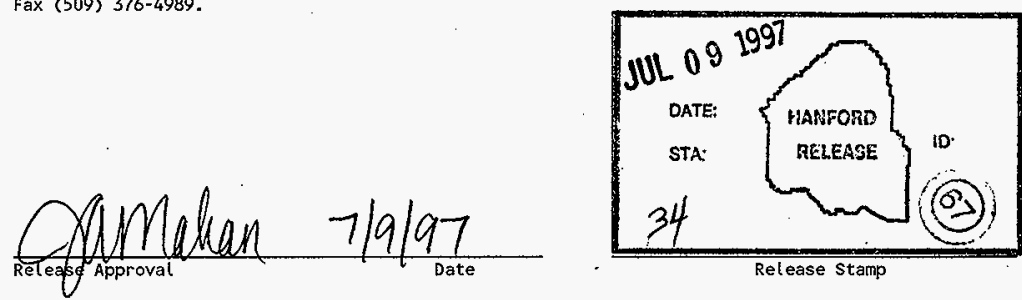

Approved for Public Release 
HNF-SD-WM-ES-411

Revision 0

\section{Engineering Study}

\section{Closure of the 209E Facility}

\section{Work Order E61945}

Prepared for the U.S. Department of Energy

Assistant Secretary for Environmental Management 
HNF-SD-WM-ES-411, Rev. 0

ENGINEERING STUDY for CLOSURE OF THE 209E FACILITY

Prepared for

Lockheed Martin Hanford Corporation by

Fluor Daniel Northwest

APPROVED:

FLUOR DANIEL NORTHWEST
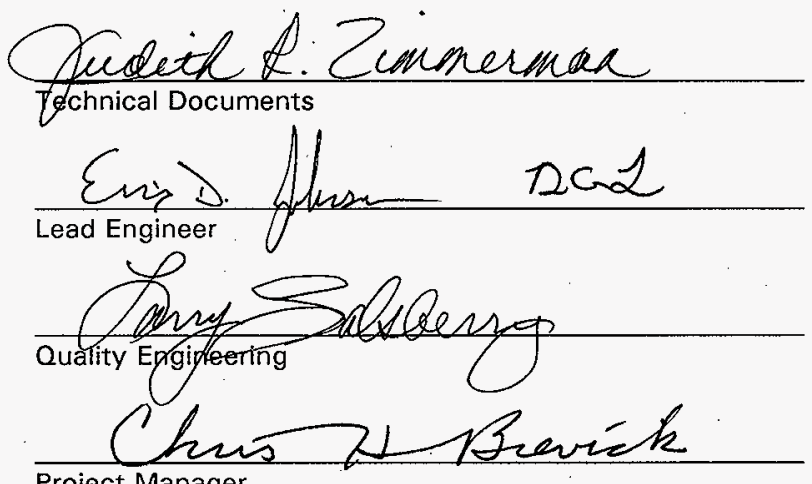

Project Manager
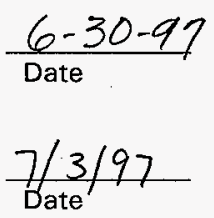

$\frac{6-30-97}{\text { Date }}$

$7 / 3 / 97$

LOCKHEED MARTIN HANFORD CORPORATION
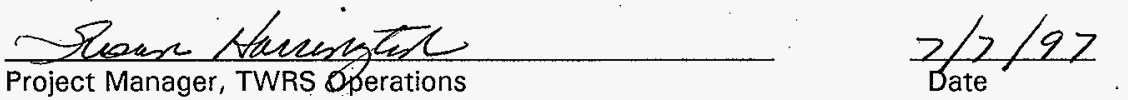


\section{ACKNOWLEDGEMENT}

The authors would like to thank the following people for their assistance in preparing this engineering study.

\section{J. L. Stroup}

Babock \&Wilcox Hanford Company

R. W. Bailey

J. P. Hayfield

W. A. Peiffer

L. D. Stefanski

Waste Management Federal Services of Hanford, Inc.

M. E. Lakes

F. C. Schmidt

M. L. Windsor

H. H. Van Tuyl, retired 


\section{TABLE OF CONTENTS}

I. INTRODUCTION ...................... 1

II. SUMMARY 'AND ÇONCLUSIONS $\ldots \ldots \ldots \ldots \ldots \ldots$

III. DESCRIPTION OF ALTERNATIVES $\ldots \ldots \ldots \ldots \ldots \ldots \ldots \ldots \ldots$

A. CRITERIA ..................... 7

B. ALTERNATIVES CONSIDERED $\ldots \ldots \ldots \ldots \ldots \ldots \ldots$

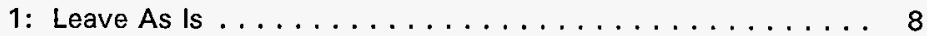

2: Fix In Place ..................... 9

3: Removal and Fix In Place ............... 13

4: Stage-Wise Removal . . . . . . . . . . . . . 17

5: Turnover to the EM-60 Program . . . . . . . . . 21

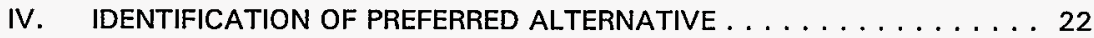

A. EVALUATION OF ALTERNATIVES $\ldots \ldots \ldots \ldots \ldots \ldots \ldots \ldots . \ldots 22$

B. PREFERRED ALTERNATIVE $\ldots \ldots \ldots \ldots \ldots \ldots \ldots$

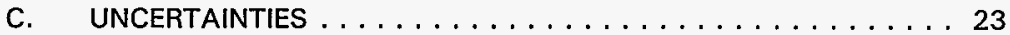

V. REQUIRED CHANGES TO IMPLEMENT PREFERRED ALTERNATIVE . . . 24

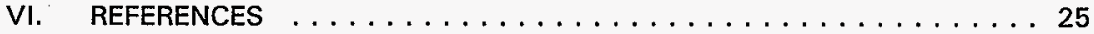

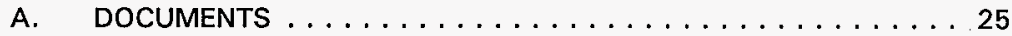

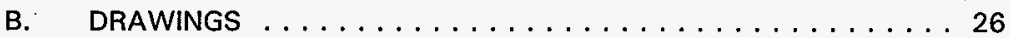

\section{APPENDICES}

Appendix A Cost Estimates

Appendix B Schedules

Appendix C Equipment List 


\section{ACRONYM AND ABBREVIATIONS LIST}

BCCAA Benton County Clean Air Authority

BHI Bechtel Hanford, Inc.

BWHC Babcock \&Wilcox Hanford Company

CAR critical assembly room

CFR

Code of Federal Regulations

CFRP

CML

consolidated fuel reprocessing program

$D \& D$

Critical Mass Laboratory

DOE

EA

decontamination and decommission

U.S. Department of Energy

Ecology

environmental assessment

EM-40

Washington State Department of Ecology

U.S. Department of Energy, Environmental Restoration

Division

EM-60 U.S. Department of Energy, Office of Facility Transition

FDNW Fluor Daniel Northwest, Inc.

FEAS

HEPA

fuel element assembly system

HVAC

high-efficiency particulate air

LMHC

NDA

heating, ventilating, and air conditioning

NEPA

Lockheed Martin Hanford Company

NOC

nondestructive analysis

PBS

$\mathrm{Pu}$

National Environmental Policy Act

Notice of Construction

PUREX

polymeric barrier system

plutonium

RCRA

$\mathrm{RL}$

Plutonium Uranium Extraction Facility

ROM

SAR

SWB

TK

Resource Conservation and Recovery Act

Richland Operations Office (DOE)

rough order of magnitude

safety analysis report

standard waste box

WIPP

tank

WRAP Waste receiving and packaging

WSDOH Washington State Department of Health 


\section{ENGINEERING STUDY \\ for \\ CLOSURE OF THE 209E FACILITY}

WORK ORDER E61945

\section{INTRODUCTION}

The Critical Mass Laboratory (CML) is located in the 200-East Area of the Hanford Site, south of 7th Street and east of Baltimore Avenue (Fig 1). The building is identified as the 209E Facility. The facility was designed to provide a heavily-shielded reactor room where quantities of fissile materials in solution could be brought into critical configurations under carefully controlled and monitored conditions (Fig 2). The facility no longer has a mission and is awaiting final closure and decontamination and decommissioning (D\&D).

The CML is not included in any Resource Conservation and Recovery Act (RCRA) Part A or B permit. National Environmental Policy Act (NEPA) documentation was not found in the Hanford Site NEPA files for the 209E Facility.

Two CML rooms identified for remediation are the Critical Assembly Room (CAR) (Fig 3) and the Mix room (Fig 4). The CAR contains contaminated equipment including 4 gloveboxes, 12 tanks, and miscellaneous vessels and bottles. Criticality experiments were conducted in the CAR. The room contains two reactor gloveboxes where the critical test assemblies are contained. The Mix room provided the necessary facilities for receiving, handling, and preparing the various forms of fissile materials during the experiments. The room contains contaminated equipment, including three gloveboxes and eight tanks. An office complex, equipment room, and changeroom are a part of the 209E Facility, but are not addressed for closure in this engineering study. 


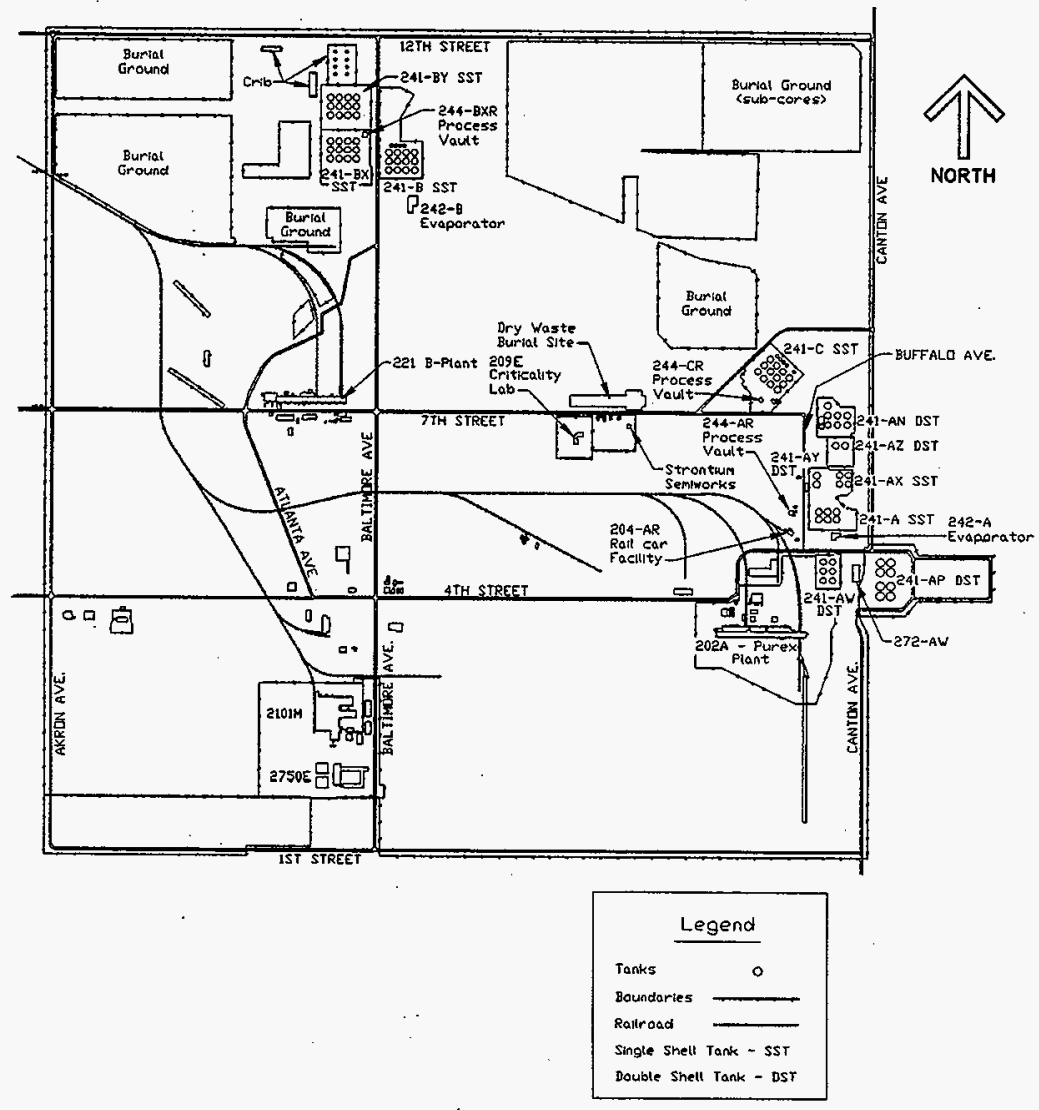

Figure 1: Hanford Site, 200-East Area 

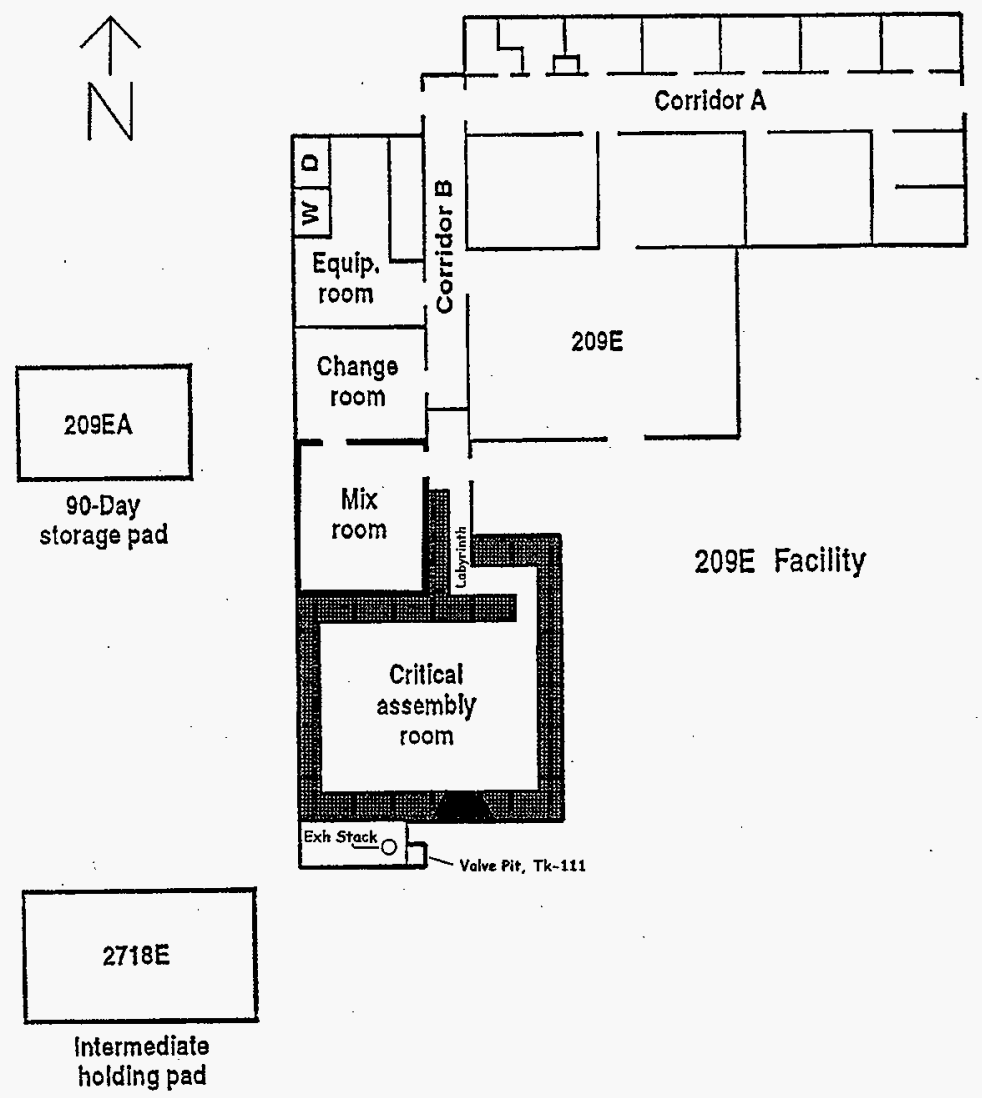

Figure 2: Critical Mass Laboratory, 209E Facility, Facility Layout 
HNF-SD-WM-ES-411, Rev. 0

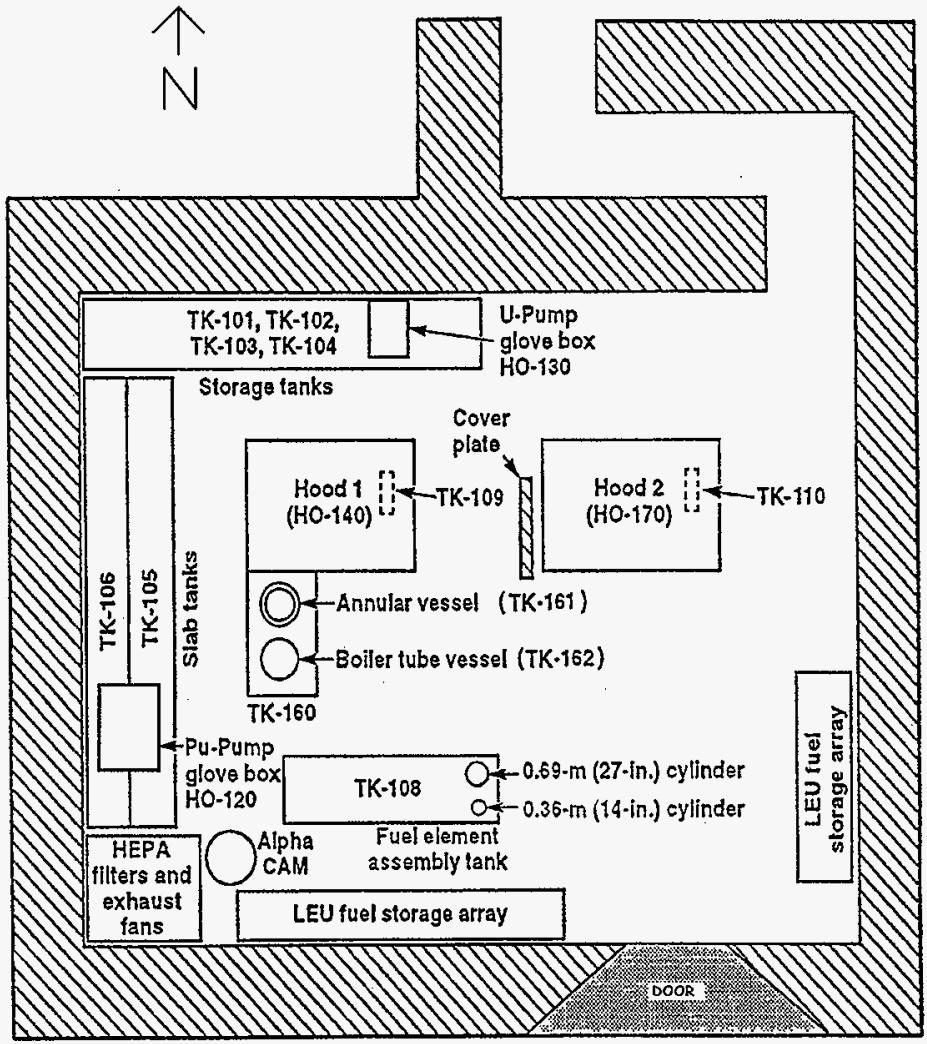

Figure 3: Critical Assembly Room Layout 


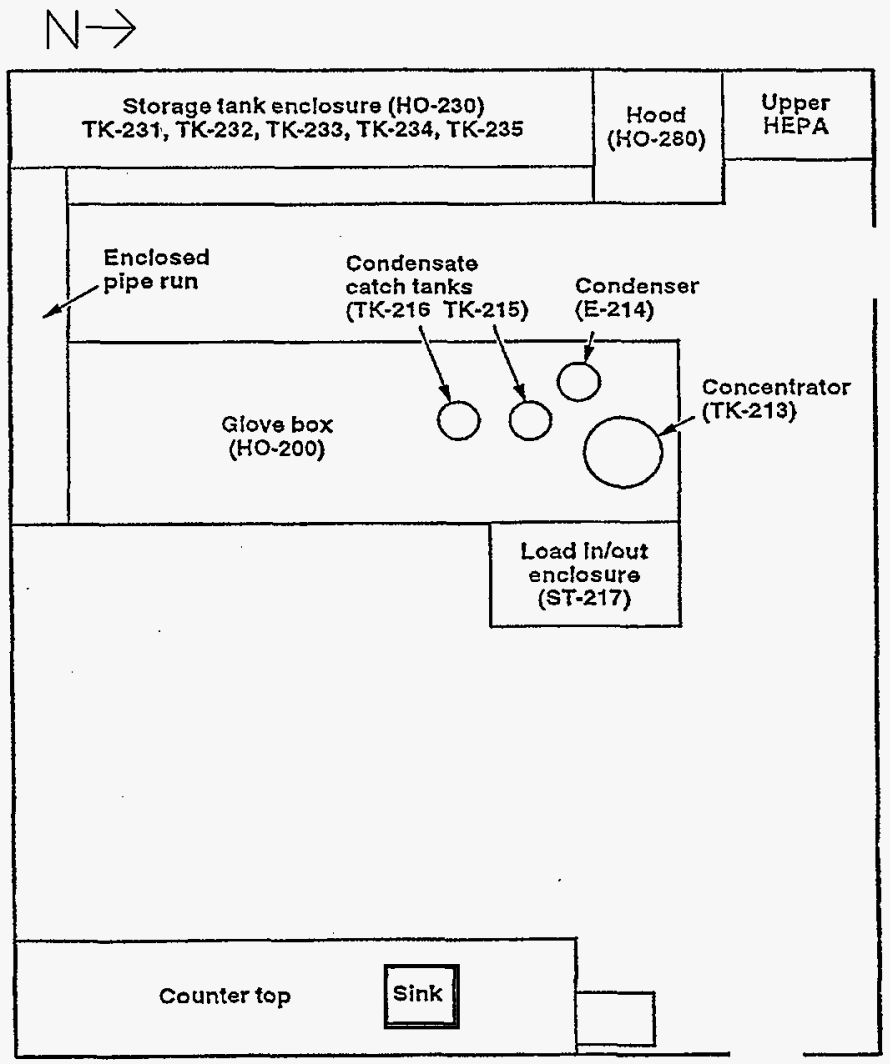

Figure 4: Mix Room Layout 
The purpose of this engineering study is to determine the safest method and most cost-effective technology to prepare the 209E Facility for closure. The engineering study will present alternatives for closure and eventual turnover of the facility from Lockheed Martin Hanford Company (LMHC) to the environmental restoration and management contractor, Bechtel Hanford, Inc. (BHI). BHI will presumably perform the D\&D of the facility under the U.S. Department of Energy (DOE) EM-40 program.

\section{SUMMARY AND CONCLUSIONS}

Five alternatives were evaluated for the closure or disposition of the 209E Facility. Table 1 summarizes the total estimated cost, manhours, and time to complete each alternative.

TABLE 1: SUMMARIZATION OF ALTERNATIVES

\begin{tabular}{|c|c|c|c|}
\hline 40 & is. & i. & 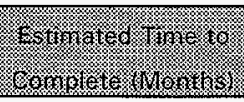 \\
\hline 1. Leave As Is & $\$ 94,188 /$ year & $1,202 /$ year & 0 \\
\hline 2. Fix In Place & $\$ 729,464$ & 8,977 & 8 \\
\hline $\begin{array}{l}\text { 3. Removal and Fix } \\
\text { In Place }\end{array}$ & $\$ 1,024,737$ & 13,327 & 10 \\
\hline $\begin{array}{l}\text { 4. Stage-Wise } \\
\text { Removal } \\
\end{array}$ & $\$ 1,721,144$ & 19,715 & 13 \\
\hline $\begin{array}{l}\text { 5. Turnover to the } \\
\text { EM-60 Program* }\end{array}$ & $\$ 10,000$ & 80 & 3 \\
\hline
\end{tabular}

* A formal cost estimate was not performed for Alternative 5 due to its minimal cost compared with the other alternatives. The estimated costs represent the cost to turnover the facility "as is" and does not include future deactivation costs to the EM-60 program.

The end-point specification document and safety analysis report (SAR) for the CML closure have not been completed, but both documents are being prepared by Babcock \& Wilcox Hanford Company (BWHC) and by LMHC. The documents are important because they explain the acceptance criteria for turnover to BHI. This study was not 
based upon $\mathrm{BHI}$ acceptance criteria, but on current technology (taking safety and cost effectiveness into account), lessons learned at the Plutonium Uranium Extraction Facility (PUREX), the 308 Building, and personnel (operators and engineers) experience.

Fluor Daniel Northwest (FDNW) recommends that future engineering studies on the closure, decommission, or shutdown of a facility consider having the end-point specification and the SAR completed prior to starting a study.

\section{DESCRIPTION OF ALTERNATIVES}

\section{A. CRITERIA}

The objective of this study is to determine the safest and most cost-effective method for closure of the CML (209E Facility) until turnover to the D\&D contractor. The strategy should be similar to that used by BWHC in prior remediation efforts used in the closure of PUREX. It is assumed that a debris removal effort, to be completed before the remediation activities begin, will address all loose waste items in the facility, i.e., trash, waste drums, miscellaneous containers of chemicals, packaged waste, etc.

The original goal was to prepare the building for closure having a nonnuclear classification of "other industrial," however, FDNW determined that this classification is not achievable considering the objective of this study. The following criteria were addressed:

- Determine the best and most cost-effective technology for cleaning and applying a fixative to the remaining plutonium (Pu).

- Investigate the safest and most current methods to decontaminate and remediate the plutonium-handling equipment (i.e., gloveboxes, fume hoods, and piping), ductwork and fixtures. Address both radiochemical and hazardous waste.

- Assess the environmental, regulatory, and permitting requirements. 


\section{B. ALTERNATIVES CONSIDERED}

A four-step approach was utilized to develop alternatives with each alternative building upon the last. While developing these approaches, an alternative evolved that did not use the same approach as the other four; this concept was added as Alternative 5. The following alternatives were considered:

Alternative 1. Leave As is

Alternative 2. Fix In Place

Alternative 3. Removal and Fix in Place

Alternative 4. Stage-Wise Removal

Alternative 5. Turnover to the EM-60 Program

Prior to shutdown of the facility, all tanks in the solution handling system were flushed with condensate to remove as much $\mathrm{Pu}$ as reasonably possible. All of the tanks were verified as empty and were purged with an air sweep to evaporate most or all of the residual liquid. During unmanned status, an air sweep of nominal 30 liters/hour (one cubic foot/hour) was maintained for all storage tanks. In any of the alternatives where tanks, vessels, etc., are to be sealed or left without decontaminating, they need to be confirmed as dry or dried. This action is necessary, especially for those tanks that are to be sealed, due to the possibility of residual water in vessels being converted, by residual radioactivity, into a mixture of hydrogen and oxygen gases (Van Tuyl 1987). This mixture of gases could possibly lead to a ruptured tank or flammable gases being released into the facility.

\section{Alternative 1: Leave As Is}

Alternative 1 would continue with the present facility operations, surveillance, and maintenance schedule. The fire protection system would remain in service; the system is wet pipe to the CAR and dry pipe inside the CAR. The CAR halon system is out of service. The estimated yearly cost for this alternative is $\$ 94,188$, with an estimated 1,202 manhours. The estimate also includes the cost of maintaining the office spaces within the facility. Appendix A, pages 1 through 12, contains the detailed cost estimate for Alternative 1. 


\section{Electrical}

The normal power, emergency power, exhaust fan, and vacuum air sampling electrical systems are still in service. All other electrical compartments are out of service. The batteries for the emergency lights have been removed. The criticality alarm system for the Mix Room has been removed and transferred to the CAR. The intercom was turned off and only one telephone service was left. The electric supply for the annunciator panel has been disconnected.

\section{Heating, Ventilating, and Air Conditioning}

The supply ventilation is shut off and air is supplied to the CAR and the Mix room by infiltration. The exhaust fan is operating below maximum capacity.

\section{Environmental}

Alternative 1 would not require permits and regulatory notifications because no remediation work would be performed on the facility.

\section{Advantages}

No additional costs above those already required would be needed to maintain the facility. Alternative 1 is the simplest of all alternatives and requires no additional work to the facility.

\section{Disadvantages}

Alternative 1 does not reduce possible health and environmental risks. It does not further the $D \& D$ process or meet the $R L$ objective of remediation of nonactive facilities.

\section{Alternative 2: Fix In Place}

Alterative 2 would fix in place all contamination with an acrylic latex contamination fixative (polymeric barrier system [PBS]) (the fixative) and shut down all building systems except for required safety systems. 


\section{Electrical}

Normal and emergency power would be turned off in the CAR and the Mix room, except for lighting, fire alarm, receptacles/outlets, air sampler, exhaust ventilation system, and constant air monitoring on the main exhaust stack. The power would be turned off and the cables disconnected and removed before the fixative is applied to the equipment. In addition, all accessible electric components associated with the equipment would be removed. After all the work is completed, the ventilation, receptacle/outlets, lighting and fire protection system would be turned off. For safety purposes, the fire protection system would be drained and disconnected last. Turning off the fire protection system is contingent on being able to zero value the building, i.e., change the building assessed value to zero dollars.

\section{Heating, Ventilating, and Air Conditioning}

Currently, the supply ventilation system is turned off and air is supplied by infiltration. The supply ventilation system may need to be restarted if the required number of room air changes cannot be met through increasing the exhaust rate and, thus, the rate of infiltration. The confinement heating, ventilating, and air-conditioning (HVAC) system would need to be reactivated.

\section{Environmental}

Since fixing the contamination would be performed in rooms with ventilation controls, there is minimal potential to emit fugitive radioactive air emissions. The Washington State Department of Health (WSDOH) must be contacted, and a Notice of Construction (NOC) may be required. If any of the areas containing asbestos would be disturbed by the work to fix the contamination, a good faith inspection must be performed and the Benton County Clean Air Authority (BCCAA) must be notified ten working days prior to work beginning.

A notice to the Washington State Department of Ecology (Ecology) is unnecessary because there is no apparent route for releasing any hazardous air emissions. A NEPA review would be used to evaluate the environmental impacts of entering the 
facility to deactivate, deenergize, and/or isolate unneeded facility systems. This is expected to be allowed by using the existing sitewide Categorical Exclusion.

\section{Description of Alternative}

The first step of Alternative 1 would be to isolate the gloveboxes/hoods. All pipe and tubing intrusions would be cut and capped outside the gloveboxes/hoods. A glove bag would be used to cover the pipes during this process. The inside of the gloveboxes/hoods would be wiped down to remove as much surface contamination as possible. Contaminated areas inside the gloveboxes/hoods would then be sprayed with the fixative. The remaining glovebox ports and hood openings would then be sealed. The glovebox ports would be sealed with a metal plate and the hoods sealed with a fabricated metal door (closure).

All gloveboxes contain a high efficiency particulate air (HEPA) filter that vents/inlets directly to the room. These HEPA filters would be removed and the opening sealed. One of the metal plates sealing the glovebox ports would be designed to house a HEPA filter mask canister to allow the gloveboxes to passively vent to their respective rooms, thus eliminating any danger of pressurizing.

The floor and walls of the CAR and the Mix room would be surveyed for areas of contamination. These areas would be wiped clean and any remaining contamination fixed in place with the fixative.

All remaining slab tanks, i.e., not located in a glovebox/hood, would be sealed shut by cutting and capping the inlet to the tanks, using the same method as cutting and capping the pipes and tubing into the gloveboxes/hoods. The only exceptions would be tank (TK) -109, TK-110, and TK-111. These slab tanks are encased in concrete, TK-109 and TK-110 are buried in concrete 1 meter $(3 \mathrm{ft})$ under the CAR while TK-111 is located in the waste hold-up valve pit outside the building, level with the floor of the pit. 
A nondestructive analysis (NDA) was not performed on these tanks due to inaccessibility. TK-109 and TK-111 are known to have contained radioactive waste, while TK-110 never received any radioactive waste but, due to its piping configuration, radioactive waste could have entered the tank either by leaks or operator error. The removal of these tanks would present more hazards to workers and the environment than leaving them intact. Therefore, it is recommended that the tanks be sealed utilizing available technologies, i.e., filled with grout for equivalent), and sealed off completely.

TK-160 (the consolidated fuel reprocessing program [CFRP] water tank) contains CFRP process tanks, TK-161 and TK-162. If TK-160 still contains water, it should be drained. TK-161 and TK-162 would then be sealed by cutting and capping the inlets and outlets, using the same technique used for the slab tanks.

TK-108, the fuel element assembly system (FEAS), contains a 360-mm (14-in.) and a 690-mm (27-in.) experimental vessel. No other information on the dimensions of these tanks could be found, therefore, the tanks would need to be examined before a containment solution could be determined.

The active exhaust ventilation systems for the CAR and the Mix room are interconnected before exiting the stack. All gloveboxes/hoods have a HEPA filter - positioned before the ventilation duct. Exhaust ventilation air from each room passes through a bank of HEPA filters. The exhaust ventilation systems connect before the first stage of HEPA filtration outside the building. The active exhaust ventilation system is likely contaminated up to the first bank of HEPA filters outside of the building. The active exhaust ventilation system would be sealed off just down stream from that HEPA filter. This allows the contaminated ducting and gloveboxes/hoods to equalize pressure with the room via the HEPA filtered passive vents in the gloveboxes/hoods. All possible egress points, i.e., aerosol/smoke injection ports and joints, would be sprayed with the fixative. 
The hoist and trolley crane located in the Mix room would be removed from service and mothballed.

Minimal maintenance and surveillance would be required until turnover to the $D \& D$ contractor. The estimated cost for Alternative 2 is $\$ 729,464$ with an estimated 8,977 manhours. Appendix A, pages 13 through 24, contains the detailed cost estimates. Appendix $B$ contains the schedule for Alternative 2.

\section{Advantages}

The advantage of Alternative 2 is that it is a quick and inexpensive solution. It is fairly simple to survey the equipment, walls, and floors and fix the contamination in place. Applying the fixative could possibly contain all contamination and, thereby, reduce the overall operating cost of the facility. In addition, an environmental assessment (EA) is not expected to be necessary.

\section{Disadvantages}

The likelihood of the fixative being able to contain all of the Pu in place is uncertain. Due to the complexity and inaccessibility inside and under/behind gloveboxes (there is believed to be contamination under some gloveboxes), there would be many areas where contamination may not be fixed in place. TK-105 and TK-106 (located in the CAR) have a history of weeping plutonium, even after attempts to seal the contamination. Attempting to isolate and seal these tanks could be unsuccessful over an extended period of time. The HEPA canister filters fitted into the ports on the gloveboxes/hoods, to be used as passive ventilation, are designed for low airflow. There is the remote possibility that the equalization airflow could exceed the filters design limit.

\section{Alternative 3:-Removal and Fix In Place}

Alternative 3 is an expansion of Alternative 2. All equipment would be removed from the gloveboxes/hoods before cleaning, applying the fixative, and sealing the gloveboxes/hoods. TK-105 and TK-106 would be removed from the facility. 


\section{Electrical}

Normal and emergency power should be turned off in the CAR and the Mix room except for lighting, fire alarm, receptacles/outlets, air sampler, exhaust ventilation system, and constant air monitoring on the main exhaust stack. When equipment is removed or sprayed with the fixative, the power would be turned off before disconnecting and removing the cables from the equipment. In addition, all accessible electric components associated with the equipment would be removed. After all the work is completed, the ventilation, receptacle/outlets, lighting, and fire protection system would be turned off. For safety purposes, the fire protection system would be drained and disconnected last. Turning off the fire protection system is contingent on being able to zero value the building.

\section{Heating, Ventilating, and Air Conditioning}

Currently, the supply ventilation system is off and air is supplied by infiltration. The supply ventilation system may need to be restarted if the required number of room air changes cannot be met through increasing the exhaust rate and, thus, the rate of infiltration. The confinement HVAC system would need to be reactivated to full functionality.

\section{Environmental}

Removing waste and equipment has the potential to emit fugitive radioactive air emissions. An NOC must be submitted to the WSDOH.

If asbestos is suspected, a good faith inspection must be performed and the BCCAA must be notified ten working days prior to work beginning. All asbestos work must comply with Code of Federal Regulations (CFR) 40 CFR 61 and the agreement with the BCCAA. Since the asbestos would be assumed to be radioactively contaminated, the material would be disposed of in burial boxes and sent to the disposal trenches in the 200-West Area.

A notice to Ecology could be necessary if hazardous air emissions from cutting up items of contaminated heavy metals would be released. 
Alternative 3 may require an EA to analyze the unknowns associated with the hazardous and radioactive materials (e.g., location and dose exposure levels in areas currently unaccessible as in and around gloveboxes and/or tanks).

Alternative 3 would produce radioactive and mixed waste requiring storage and/or disposal.

\section{Description of Alternative}

The first step of Alternative 3 would remove (bag out) all equipment (tanks, pumps, condensers, etc.) in the existing gloveboxes/hoods. Equipment must fit into a waste isolation pilot plant (WIPP) certified container. The equipment would need to be cut into appropriate sized pieces before removal from the glovebox/hood. The pieces would be double bagged with sharp corners and edges taped to prevent ripping of the bags and placed in standard waste boxes (SWB) or 210-L (55-gal.) drums. The SWBs and drums would then be sent to the waste receiving and packaging (WRAP) facility for later permanent disposal at the WIPP.

The gloveboxes/hoods would be decontaminated, sealed, and passively vented in the same manner as Alternative 2. The floors and walls of the CAR and the Mix room would be decontaminated in the same manner as in Alternative 2.

All slab tanks, except TK-105 and TK-106, would be sealed in the same manner as in Alternative 2. TK-105 and TK-106, are too large to place in a glovebox/hood and have a history of weeping plutonium at the south end of the tanks. Attempts to stop the leaks by caulking or painting have been successful for only a short time and had to be repeated as necessary. Attempting to isolate and seal these tanks could be unsuccessful over an extended period of time. Therefore, a greenhouse would be erected and the tanks cut up and placed in SWBs or 210-L drums.

The remaining tanks, piping, tubing, instrumentation, vessels, and ventilation systems would be handled in the same manner as Alternative 2 . The hoist and 
trolley crane located in the Mix room would be removed from service and mothballed.

Minimal maintenance and surveillance would be required until turnover to the $D \& D$ contractor. The estimated cost for Alternative 3 is $\$ 1,024,737$ with an estimated 13,327 manhours. Appendix A, pages 25 through 38 , contains the detailed cost estimates. Appendix B contains the schedule for Alternative 3.

\section{Advantages}

The main advantage of Alternative 3 over Alternative 2 is the removal of equipment from the gloveboxes/hoods and the removal of TK-105 and TK-106. Removing the equipment from the gloveboxes/hoods would allow for better decontamination of the gloveboxes/hoods, thus, lowering the amount of residual plutonium in the facility. With the gloveboxes/hoods cleaner and the remaining contamination stabilized, the concerns with the passively venting HEPA filter (as described in the disadvantages section of Alternative 2) are lessened.

By removing TK-105 and TK-106, the leaking contamination from this source is eliminated.

\section{Disadvantages}

Only a portion of the Pu source term would be removed. There is still the possibility that contamination exists under the gloveboxes/hoods that would not get fixed in place. By not removing/moving the gloveboxes/hoods in the CAR, it is not possible to be sure that all contamination is removed or fixed in place.

As in Alternative 2, the HEPA canister filters fitted into the ports on the gloveboxes/hoods, to be used as passive ventilation, are designed for low airflow. There is the remote possibility that the equalization airflow could exceed the filters design limit. Although, with the gloveboxes/hoods being cleaner in Alternative 3, the concern is lessened. 


\section{Alternative 4: Stage-Wise Removal}

Alternative 4 differs from Alternative 3 in that it removes all major equipment, most minor equipment, and cleans or fixes in place any remaining contamination. Work would take place in a stage-wise manner. Equipment with larger concentrations of materials are removed first.

\section{Electrical}

Normal and emergency power would be turned off in the CAR and the Mix room, except for lighting, fire alarm, receptacles/outlets, air sampler, exhaust ventilation system, and constant air monitoring on the main exhaust stack. When equipment is removed, the power would be turned off before disconnecting and removing the cables from the equipment. All accessible electric components associated with the equipment would be removed. After the work in Alternative 4 is completed, the ventilation, receptacle/outlets, lighting, and fire protection system would be turned off. For safety purposes, the fire protection system should be drained and disconnected last. Turning off the fire protection system is contingent on being able to zero value the building.

\section{Heating, Ventilating, and Air Conditioning}

Currently, the supply ventilation system is off and air is supplied by infiltration. The supply ventilation system may need to be restarted if the required number of room air changes cannot be met through increasing the exhaust rate and, thus, the rate of infiltration. The confinement HVAC system would need to be reactivated to full functionality.

\section{Environmental}

Removing waste and equipment has the potential to emit fugitive radioactive air emissions. An NOC must be submitted to the WSDOH.

If asbestos is suspected, a good faith inspection must be performed and the BCCAA must be notified ten working days prior to work beginning. All asbestos work must comply with 40 CFR 61 and the agreement with the BCCAA. Since the asbestos 
would be assumed to be radioactively contaminated, the material would be disposed of in burial boxes and sent to the disposal trenches in the 200-West Area.

A notice to Ecology could be necessary if hazardous air emissions from cutting up items of contaminated heavy metals would be released.

Alternative 4 would probably require an EA to analyze the unknowns associated with the hazardous and radioactive materials (e.g., location and dose exposure levels in areas currently unaccessible as in and around gloveboxes and/or tanks).

Alternative 4 would produce radioactive and mixed waste requiring storage and/or disposal.

\section{Description of Alternative}

All equipment is required to be disposed of in a WIPP certified container, either a 210-L drum or an SWB. Equipment too large for either of these containers would be size reduced. Equipment pieces would be double wrapped in plastic with any sharp edges or protrusions covered with tape to protect the plastic from cuts. All containers would be processed through the WRAP facility for certification and stored for eventual shipment to WIPP for disposal.

\section{Stage 1:}

The first activity for equipment removal would be to isolate the equipment from all systems except any required safety systems. Piping and tubing systems would be cut and capped using the same method as in Alternatives 2 and 3. The next step would to remove all accessible equipment within the glovebox and package it for disposal. Most glovebox internal equipment should not require size reduction, except for some of the slab tanks. Removal of equipment would include disassembly and cutting to reduce size. Once internal equipment is removed and the gloveboxes are isolated, the inside and outside if required, would need to be painted with the fixative to provide contamination control in preparation for size reduction. The final step prior to size reduction would be to construct a ventilated greenhouse 
around the glovebox and disconnect any remaining safety systems and the active ventilation system.

The first step in size reducing the old style gloveboxes would be to remove the plexiglass windows. The remaining framework would be cut up into manageable pieces.

Tanks would be isolated and removed for disposal. If size reduction is required, the fixative would be applied to accessible contaminated areas. When there are contaminated areas that are not accessible with the fixative, the size reduction would need to be performed inside a ventilated greenhouse. When this stage is completed, the majority of the plutonium would have been removed (an actual percentage of removal is not possible because the total plutonium held in the facility is not known, i.e some equipment and places could not be analyzed by nondestructive analysis).

The estimated cost for the first stage of Alternative 4 is $\$ 1,266,891$ with an estimated 14,427 manhours. Appendix $A$, pages 39 through 51 , contain the detailed cost estimate.

\section{Stage 2:}

The next logical step would be to remove the other piping and equipment that is known or highly suspected of containing high levels of contamination. Equipment would include the exhaust ventilation system up to the first stage of HEPA filtration outside of the building. Suspect piping and tubing would include the transfer lines, vent lines, and vacuum lines.

Transfer lines have been directly exposed to high levels of contamination and are a prime target for removal. Vessel vent lines are not directly exposed but may contain contamination due to migration or the venting of contaminated air. The exhaust outlet from the gloveboxes and hoods were not originally protected with HEPA filters. The exhaust ventilation system up to the first stage of HEPA filtration 
outside the building is most likely contaminated. Therefore, the last step of stage 2 would remove the CAR and the Mix room exhaust ventilation system up to the first stage of HEPA filtration outside the building.

The estimated cost for the second stage of Alternative 4 is an additional $\$ 99,165$ with an estimated 1,004 additional manhours. Appendix A, pages 52 through 63 , contains the detailed cost estimate.

\section{Stage 3:}

Stage 3 would include removal of all remaining equipment, cleaning and applying a fixative to contaminated concrete, and removing the remaining confinement ventilation system (ducts, filters and fans). Once the work performed by stages 1 and 2 is completed, all the remaining piping, wiring, and instrumentation can be removed from the CAR and the Mix room. This activity would leave bare walls exposed that may contain embedded contamination. However, contamination is unlikely due to the application of a fiber glass reinforced resin surface (Amercoat No. 74) that was applied to all the interior concrete surfaces of the CAR and to the concrete walls and plaster ceiling in the Mix room during initial construction. After a radiological survey determines the location of contamination on the walls and floor, the areas would be wiped clean of any contamination not removed by wiping and would be painted over. The hoist and trolley crane located in the Mix room would be removed from service and mothballed. The remaining task would be to remove the Zone 2 confinement HVAC system in its entirety.

The estimated cost for stage 3 of Alternative 4 is an additional $\$ 355,088$ with an estimated 4,284 additional manhours. Appendix A, pages 64 through 76 , contains the detailed cost estimate.

Minimal maintenance and surveillance would be required until turnover to the $D \& D$ contractor. The estimated total cost for Alternative 4 is $\$ 1,721,144$ with an estimated total of 19,715 manhours. Appendix B contains the total schedule to complete Alternative 4. 


\section{Advantages:}

The majority of the contamination source term would be removed, thereby, reducing the possibility of exposure to people and the environment. Work performed in Alternative 4 helps future D\&D operations on the facility.

\section{Disadvantages:}

It is unlikely that the facility would be clean enough to be classified as "other industrial." The application of Alternative 4 is very costly and more removal than is necessary by any subsequent $D \& D$ activity may be performed.

\section{Alternative 5: Turnover to the EM-60 Program}

Alternative 5 would turn the CML over to the DOE EM-60 program, managed by $B W H C$, for use as a D\&D training facility and a site for testing new $D \& D$ technologies.

After a general housekeeping is performed, Alternative 5 would leave the facility as is and the CML would be turned over to the EM-60 program. The EM-60 program would be responsible for continued maintenance, surveillance, and operation of the facility and grounds. A formal cost estimate was not performed for Alternative 5 due to its relatively low cost compared to the other alternatives. However, a rough order of magnitude (ROM) cost estimate for Alternative 5 is $\$ 10,000$ with an estimated 80 manhours mainly for administrative work to turn over the facility. This cost does not include the EM-60 program deactivation costs.

Communications are underway with BWHC, LMHC, and RL regarding the transfer of the 209E Facility to BWHC for use as a training facility.

\section{Advantages}

The CML is an isolated self-contained laboratory facility with office space, meeting rooms, a control room, and a changeroom that provides a good setting for training. There are rooms for additional training needs and sufficient parking around the building. 
The CAR and the Mix room contain typical gloveboxes and equipment that would be found in other DOE facilities that handled fissile materials. The CML would be an ideal training center for teaching D\&D workers the techniques required for safe and efficient $D \& D$ operations. It would also provide an ideal facility to test new $D \& D$ systems. New decontamination methods and size reduction equipment could be tested in the facility.

\section{Disadvantages}

Although contamination levels are not excessive, it would still be a challenge to decontaminate to non-transuranic levels and to provide a realistic evaluation of the methods effectiveness.

\section{IDENTIFICATION OF PREFERRED ALTERNATIVE}

\section{A. EVALUATION OF ALTERNATIVES}

Alternative 1, Leave As Is, was ruled out as a viable alternative early in the study because it did not meet RL objectives for closure of nonactive facilities. LMHC also expressed their disinterest in this alternative.

Alternative 2, Fix In Place, is one of the more cost effective alternatives, but it does not leave the building in the safest state for personnel and the environment. In a less complex facility, this would be a good solution but it does not meet the needs of the CML.

Alternative 3, Removal and Fix In Place, mitigates the primary concerns encountered in Alternative 2 by removing the equipment in the gloveboxes/hoods and the leaking tanks. Although costs for Alternative 3 are approximately $29 \%$ more than costs for Alternative 2, the facility would be left in a safer state for personnel and the environment.

Alternative 4, Stage-Wise Removal, is the most extensive and thorough of all the alternatives, but is also the most expensive. While this alternative aides future $D \& D$ 
work, it most likely performs more work than is necessary to close the facility. Alternative 4 leaves the facility in a safer state for personnel and the environment than the other alternatives, but does not balance the increase in cost, nearly $60 \%$ more than Alternative 3.

Alternative 5, Turnover to the EM-60 Program, is the most cost effective of all alternatives. The alternative does not include any decontamination but turning the facility over to the EM-60 program would result in the facility receiving decontamination via training and testing of new technologies. This is a win-win solution: the building would eventually receive the needed D\&D work, a facility would be provided to train workers, and new D\&D technologies could be developed.

\section{B. PREFERRED ALTERNATIVE}

Alternative 5 is the preferred alternative. It is the most cost effective alternative and maintains the building in a safe state. Hanford and other DOE sites would benefit by implementing this alternative. D\&D workers would be trained in new and safer techniques, and new D\&D technologies would be developed that may save lives and tax payer dollars in the future.

To execute Alternative 5, RL and LMHC would have to resolve the best course of action to achieve turning over the facility to the EM-60 program. A second alternative was chosen if $R L$ determines that Alternative 5 is not a viable solution. The second preferred alternative is Alternative 3 , Remove and Fix In Place, because it presents the best balance of safety and cost effectiveness of the other four alternatives.

\section{UNCERTAINTIES}

The only uncertainty is if RL will decide that turnover of the facility to the EM-60 program is a viable solution. Therefore, a backup alternative is presented.

The uncertainties in Alternative $\mathbf{3}$ are whether there is contamination under the gloveboxes in the CAR and if sealing the underground tanks with grout would prove 
satisfactory. While contamination under the gloveboxes is not a safety problem while the building is idle, it becomes an unknown for D\&D workers. There is some work being performed in Savannah River with "smart grout" that may work better for filling the underground tanks. This process should be considered if Alternative 3 is chosen.

\section{REQUIRED CHANGES TO IMPLEMENT PREFERRED ALTERNATIVE}

Alternative 5, Turnover to the EM-60 Program, would require the following activities:

- Resolution of the current unresolved safety question, specific to contamination control.

- Completion of the facility SAR.

- Completion of the facility housekeeping and waste disposal effort.

- Completion of a property and equipment transfer form.

- Financial transfer of current and future operating funds.

Alternative 3, Removal and Fix In Place, would require the following actions:

- Resolution of the current unresolved safety question, specific to contamination control.

- Completion of the facility SAR.

- Completion of the facility housekeeping and waste disposal effort. 


\section{REFERENCES}

\section{A. DOCUMENTS}

Byrd, W.H.; March 1959; Specifications for Critical Mass Laboratory Building 209-E; HWS-6471; General Electric Company, Richland, Washington.

Divine, J.R.; November 1988; CML Tube Pits; Internal Memo; Pacific Northwest Laboratories, Richland, Washington.

Dodd, Edwin III; January 1997; Preliminary Hazards Category Evaluation For Possible End State Conditions at 244AR Vault and 209E Facilities; Link Technologies, Incorporated, Richland, Washington.

Flowers, C.D.; December 1988; Project D-370 Tubing Failure; Internal Letter; Pacific Northwest Laboratories, Richland, Washington.

Hamrick, D.G. and M.S. Gerber; September 1996; PUREX/UO $\mathrm{O}_{3}$ Facilities Deactivation Lessons Learned History; WHC-SP-1147, Rev. 1; Westinghouse Hanford Company, Richland, Washington.

Lakes, M.E.; October 1996; Preliminary Hazards Analysis, 209-E Bldg, Critical Mass Laboratory; WHC-SD-WM-TI-789, Rev. 0; Westinghouse Hanford Company, Richland, Washington.

Metcalf, I.L.; August 1993; 308 Building Shutdown Plan; WHC-SD-FL-SSP-001, Rev. 1; Westinghouse Hanford Company, Richland, Washington.

Morton, M.R.; March 1997; Facility Transition Instruction; BHI-00961, Rev. 1; Bechtel Hanford Company, Richland, Washington.

Peterson, R.S.; November 1958; General Specification for Glove Type Hoods; HWS-6480; General Electric Company, Richland, Washington. 
Radiological Survey Report; WH-001, Rev. 009; Westinghouse Hanford Company, Richland, Washington.

Reardon, W.A., et. al; August 1960; Hazards Summary Report for the Hanford Plutonium Critical Mass Laboratory; HW-66266; General Electric Company, Richland, Washington.

Status Report for Hanford's Critical Mass Laboratory, Rev. O; February 1989; Pacific Northwest Laboratories, Richland, Washington.

Tuyl, T.D.; March 1991; Fixing Contamination at the Critical Mass Laboratory; Internal Letter; Pacific Northwest Laboratories, Richland, Washington.

Wilson, B.; February 1996; Dangerous Waste Compliance Inspection at the $209 E$ Facility on September 15, 1995; Letter; State of Washington Department of Ecology, Kennewick, Washington.

\section{B. DRAWINGS}

H-2-32570, Rev. 2; 1985; Bellows Tank; General Electric Company, Richland, Washington.

H-2-32567, Rev. 3; 1988; Mix Dump \& Storage Tank; General Electric Company, Richland, Washington.

H-2-33854, Rev. 1; 1987; Alternate Solution Storage Tank TK-103 \& TK-104; Pacific Northwest Laboratories, Richland, Washington.

H-2-33856, Rev. 2; 1987; CFRP Assembly; Pacific Northwest Laboratories, Richland, Washington.

H-2-44344, Rev. 4; 1981; Exhaust System Critical Assembly \& Mix rooms; General Electric Company, Richland, Washington. 
H-2-44357, Rev. 3; 1960; Reactor Hood Assembly; General Electric Company, Richland, Washington.

H-2-44358, Rev. 3; 1960; Reactor Hood Frame; General Electric Company, Richland, Washington.

H-2-44359, Rev. 3; 1960; Reactor Hood Misc. Details; General Electric Company, Richland, Washington.

H-2-44361, Rev. 2; 1960; Reactor Hood Dump Tank; General Electric Company, Richland, Washington.

H-2-44371, Rev. 5; 1983; Process Area Flow Diagram; General Electric Company, Richland, Washington.

H-2-44373, Rev. 4; 1988; HO-130 U Pump Glovebox Assembly \& Details; Pacific Northwest Laboratories, Richland, Washington.

H-2-44380, Rev. 2; 1960; Critically Safe Crib Waste Hold-up Tank; General Electric Company, Richland, Washington.

H-2-95713, Rev. 1; 1985; Process Piping Engineering Flow Diagram; Pacific Northwest Laboratories, Richland, Washington.

H-2-95719, Rev. 1; 1988; HO-140 Assembly Hood \#1 Process Piping; Pacific Northwest Laboratories, Richland, Washington.

H-2-95723, Rev. 2; 1985; HO-170 Assembly Hood \#2 Process Piping; Pacific Northwest Laboratories, Richland, Washington.

H-2-95754, Rev. 1; 1988; Fire Protection Sprinkler Sys, Zone Plan; Pacific Northwest Laboratories, Richland, Washingtion. 
H-2-95757, Rev. 3; 1988; TK-101 \& TK-102 Solution Storage Tanks; Pacific Northwest Laboratories, Richland, Washington.

H-2-95758, Rev. 2; 1988; Metallic Bellows; Pacific Northwest Laboratories, Richland, Washington.

H-2-96013, Rev. 2; 1986; Flow Diagram Composite Crit Assy Room; Pacific Northwest Laboratories, Richland, Washington.

H-2-96014, Rev. 2; 1986; Piping \& Instrument Diagram TK-108 \& TK-111; Pacific Northwest Laboratories, Richland, Washington.

H-2-96015, Rev. 2; 1986; Piping \& Instrument Diagram HO-170; Pacific Northwest Laboratories, Richland, Washington.

H-2-96016, Rev. 2; 1986; Piping \& Instrument Diagram HO-14O; Pacific Northwest Laboratories, Richland, Washington.

H-2-96017, Rev. 2; 1986; Piping \& Instrument Diagram TK-160; Pacific Northwest Laboratories, Richland, Washington.

H-2-96018, Rev. 2; 1986; Piping \& Instrument Diagram HO-12O; Pacific Northwest Laboratories, Richland, Washington.

H-2-96019, Rev. 2; 1986; Piping \& Instrument Diagram TK-105 \& TK-106; Pacific Northwest Laboratories, Richland, Washington.

H-2-96020, Rev. 2; 1986; Piping \& Instrument Diagram HO-130; Pacific Northwest Laboratories, Richland, Washington.

H-2-96021, Rev. 2; 1986; Piping \& Instrument Diagram TK-101 \& TK-102; Pacific Northwest Laboratories, Richland, Washington. 
H-2-96022, Rev. 2; 1986; Piping \& Instrument Diagram TK-103 \& TK-104; Pacific Northwest Laboratories, Richland, Washington.

H-2-96023, Rev. 2; 1986; Piping \& Instrument Diagram HO-200; Pacific Northwest Laboratories, Richland, Washington.

H-2-96024, Rev. 2; 1986; Piping \& Instrument Diagram HO-230; Pacific Northwest Laboratories, Richland, Washington.

H-2-96032, Rev. 1; 1987; HO-230 Storage Tank Enclosure; Kaiser Engineers Hanford, Richland, Washington.

H-2-96033, Rev. 0; 1985; HO-230 Piping \& Stor Tanks Instl Details; Kaiser Engineers Hanford, Richland, Washington.

H-2-96041, Rev. 0; 1985; Equipment Arrangement HO-200 Mix room Glovebox Plan/Section; Kaiser Engineers Hanford, Richland, Washington.

H-2-96043, Rev. 0; 1985; Piping HO-200 Mix room Glovebox Plans; Kaiser Engineers Hanford, Richland, Washington.

H-2-96046, Rev. 0; 1985; Vessel Assembly TK-206 Prover Vessel; Kaiser Engineers Hanford, Richland, Washington.

H-2-96047, Rev. 0; 1985; Vessel Assembly TK-213 Concentrator; Kaiser Engineers Hanford, Richland, Washington.

H-2-96048, Rev. 0; 1985; Vessel Assembly TK-242 Closed Loop Expansion Tank; Kaiser Engineers Hanford, Richland, Washington.

H-2-96049, Rev. 0; 1985; Vessel Assembly TK-215 and TK-216 Process Cnds Catch $T k$; Kaiser Engineers Hanford, Richland, Washington. 
H-2-96050, Rev. 0; 1985; Vessel Assembly TK-231, 232 \& 233 Pu Storage Tanks; Kaiser Engineers Hanford, Richland, Washington.

H-2-96051, Rev. 0; 1985; Vessel Assembly TK-234 \& 235 Pu Storage Tanks; Kaiser Engineers Hanford, Richland, Washington.

H-2-96052, Rev. 0; 1985; Piping HO-120 Pu Pump Glovebox Plan \& Sections; Kaiser Engineers Hanford, Richland, Washington.

H-2-96054, Rev. 0; 1985; Piping HO-140 Modifications Critical Assembly Hood \#1; Kaiser Engineers Hanford, Richland, Washington.

H-2-96057, Rev. 0; 1985; Equipment Arrangement Critical Assy, Mix \& Control Rooms; Kaiser Engineers Hanford, Richland, Washington.

H-2-96060, Rev. 1; 1987; Glovebox Assembly \& Installation HO-200; Kaiser Engineers Hanford, Richland, Washington.

H-2-96071, Rev. 0; 1985; Plutonium Pump Glovebox Assembly HO-120; Kaiser Engineers Hanford, Richland, Washington.

H-2-96072, Rev. O; 1986; Piping \& Instrument Diagram HVAC Supply \& Process Exh; Pacific Northwest Laboratories, Richland, Washington.

H-2-96073, Rev. 0; 1985; HVAC Plans \& Schedules; Kaiser Engineers Hanford, Richland, Washington.

H-2-96074, Rev. 0; 1985; HVAC Sections \& Details, Kaiser Engineers Hanford, Richland, Washington.

H-2-96085, Rev. 0; 1985; Instm Eqpt Arr Plan Critical Assy Room, Kaiser Engineers Hanford, Richland, Washington. 
HNF-SD-WM-ES-411, Rev. 0

\section{APPENDIX A}

\section{Cost Estimates}

Page

Alternative 1 : Leave As Is . . . . . . . . . . . . . A-1

Alternative 2: Fix In Place $\ldots \ldots \ldots \ldots \ldots \ldots \ldots \ldots \ldots \ldots \ldots$

Alternative 3: Removal and Fix In Place $\ldots \ldots \ldots \ldots \ldots \ldots \ldots \ldots$ A-25

Alternative 4: Stage-Wise Removal

Stage $1 \ldots \ldots \ldots \ldots \ldots \ldots \ldots \ldots$. . . . . . . . . . . .

Stage $2 \ldots \ldots \ldots \ldots \ldots \ldots \ldots \ldots \ldots \ldots \ldots \ldots \ldots \ldots \ldots$

Stage $3 \ldots \ldots \ldots \ldots \ldots \ldots \ldots \ldots$. . . . . . . . . . 
FLUOR DANIEL NORTHHEST, INC. LOCKHEED MART IN HANFORO CORP. JOB NO. E61945/F3RULS

TEST - INTERACTIVE ESTIMATING

FILE NO. 2475 AAB 1 209E FACILITY ERGIHEERING STUDY PLANNING PHACRO1. PROJECT COST SUMMARY
PAGE 1 OF 9

13:04:01

DKH/RHO

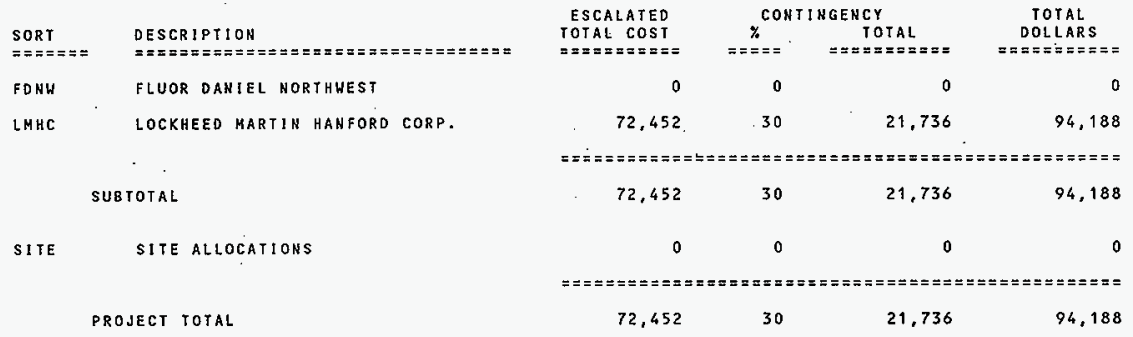

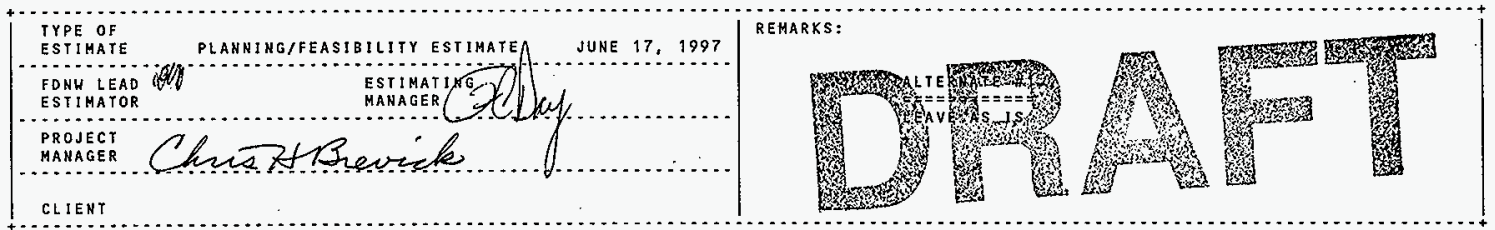


FLUOR DANIEL NORTHWEST, INC: LOCKHEED MARTIN HANFORD CORP.

JOB NO. E6IOASAF3RULS

HBS DESCRIPTION

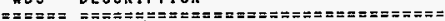

310100 alternative 1 - LEAVE as IS

PROJECT TOTAL
* * IEST - InTERACTIVE ESTIHATING **

209E FACILITY ENGINEERING STUDY

PLANNIHG/FEASIBIL ITY ESTIMATE - STERHATIVE PLANNIHG/FEASIBIL ITY ESTIMATE - ALTERNATIVE
PAGE 2 OF 9

$\begin{array}{lll}\text { PAGE } & 2 \text { OF } 9 \\ \text { DATE } & 06 / 17 / 97 & 13: 04: 06\end{array}$

DATE OK/Y TY97

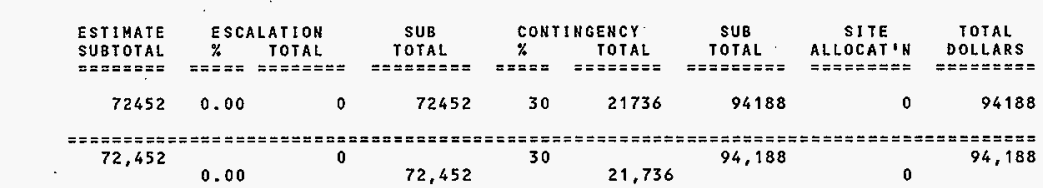


FLUOR DANIEL MORTHHEST, INC, LOCKHEED MARTIN HANFORD CORP. JOB NO. E61945/F3RULS

FILE NO. Z475AAB 1
** IEST - INTERACTIVE ESTIHATING * * 209E FACILITY ENGIHEERING STUDY PLANHING/FEASIBILITY ESTIMATE
PHMCRO3. ESTIMATE BASIS SHEET
PAGE 3 OF 9

14:17:07

1. ESTIMATE PURPOSE

THIS ESTIMATE HILL BE USED FOR A SCOPING STUDY.

2. ESTIMATE TECHNICAL BASIS

A. THIS ESTIMATE HAS BEEN PREPARED FOR LOCKHEED MARTIN HANFORD CORP AS REQUESTED BY FNDH PROJECT MANAGEMENT.

B. A ROUGH DESCRIPTION OF THE SCOPE OF WORK MAY BE FOUND IN THE OUTLINE OF ALTERNATIVES, AN UNDATED AND

A ROUGH DESCRIPTION OR

C. IHE FOLLOUING CONSTRAINTS AND/OR SPECIAL CONDITIONS EXIST: ALL WORK DONE IN THE CRITICAL ASSEMBLY ROOM AND IN THE MIXING ROOM HILL REQUIRE FOLLOWING THE APPROPRIATE RADIATION WORK PROCEDURE.

3. ESTIMATE METHODOLOGY

A. DIRECT COSTS:

A MANLOADIMG TECHNIOUE WAS USEO TO COME UP WITH THE LABOR HOURS HHEN NECESSARY DUE TO THE UNDEFINED SCOPE.

(1) CONSTRUCTIOH LABOR. MATERIAL AND EQUIPHENT UNITS HAVE BEEH ESTIMATED BASED UPON ONE OR MORE OF THE FOLLOHANG STANDARO COMMERCIAL ESTIMATING RESOURCES, PUBLISHED ESTIMATING MANUALS AND R.S. MEANS

B. DIRECT COST FACTORS

(1) SALES TAX HAS BEEN APPLIED TO ALL MATERIALS AND EQUIPMENT PURCHASES AT $8 \%$

(2) NO HAREHOUSING COSTS ARE SHOWN SINCE THEY ARE CONSIOERED TO BE INCLUDED IN THE MATERIAL PROCUREMEHT RATE (MPR S.

(3) AN ESTIMATING FACTOR OF 15\% HAS BEEN APPLIED TO DIRECT CRAFY LABOR FOR GENERAL REQUIREMENTS AND $23.58 \%$ FOR TECHHICAL SERVICES

(4) CONSUMABLES ARE ESTIMATED AT $3.2 \%$ OF DIRECT CRAFT LABOR COSIS

(5) SPECIAL HORK PROCEDURE (SWP) FACTORS ARE APPLIED AGAINST DIRECT LABOR FOR ACTUAL TIME LOST DUE TO THE PERSONNEL PROTECTIVE EQUIPMENT AND PROCEDURES. THE RAIES HHICH YAVE BEEN APPLIED ARE AS FOLLOWS

PROTECTIVE CLOTHING FACTOR HAS BEEN APPLIED AT 15\%,

MASK HORK AT $85 \%$ PLUS ' $15 \%$ FOR PROTECTIVE CLOTHING.

CONTAMINATION RESTRICTIONS ASSOCIATED WITH RADIATION DOSE LIMITS (BURNOUT) HAVE NOT BEEN CALCULATED AS IT IS NOT BELIEVE TO BE a CONCERN FOR THESE ACTIVITIES.

(6) PREMIUM PAY

OVERTIME REQUIREMENTS AHO SHIFT DIFFERENTIAL PAY FOR CRAFT LABOR WILL NOT BE REQUIRED FOR THIS WORK, AS STRAIGHT TIME, 40 HOUR WEEKS ARE DEEMED ADEQUATE TO ACCOMPLISH THIS WORK.

(7) GENERAL FOREMAN FACTOR OF 7\% HAS BEEN APPLIED TO DIRECT CRAFT LABOR CREWS ,

(8) A FACTOR OF $10 \%$ HAS BEEN APPLIED TO DIRECT CRAFT LABOR TO ALLOW FOR USAGE OF GOVERNMENT ONHEO EQUIPHENT CONTROLLED BY DYNCORP.

c. RAJES

(1) FLUOR DANIEL NORTHLEST LABOR RATES ARE BASED UPON THE FLUOR DAINEL FEDERAL OPERATIONS (FEDFO) DISCLOSURE STATEMENT AND APPROVED PROVISIONAL BILLING RATES. FOR ESTIMATING PURPOSES, AVERAGE RATES BY OPERATIONS CODE HAVE BEEN DEVELOPED BASED UPON RECENT COST HISTORY.

(2) FLUOR DAHIEL NORTHUEST SERVICES (CONSTRUCTION CRAFT LABOR) RATES ARE THOSE LISTED IN APPENDIX A TO THE HANFORD SITE

(3) FOH \& PHMC SUBCONTRACTOR STANDARO LABOR RATES ARE IHOSE LISTED IN THE FIMANCIAL DATA SYSTEM (FDS) FDST 32 TR REPORT ORGANIZATION RATES PLUS ADDERS. 
FLUOR DANIEL NORTHHEST, INC. LOCKHEED MARTIN HANFORD CORP. JOB NO. E61945/F3RULS

FILE NO. $2475 A$ AB 1

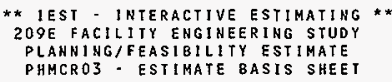

PAGE 4 OF 9

DATE $06 / 16 / 97 \quad 14: 17: 07$

BY DKH/RWO

D. SITE ALLOCATIONS FACTORS

ARE DEVELOPED AND PROVIDED BY FLUOR DANIEL HANFORO (FDHS FOR ESTIMATING USE.

(1) GOVERNMENT FURHISHED SERVICES RATE IS APPLIEO TO ALL COSTS TO LIQUIDATE GOVERNMENT FURNISHED SERVICES PROVIDED TO THE ENTERPRISE COMPANIES: 14\% FOR. FONW, TO\% FOR FONUS (CONSTRUCTION)

2) HANFORD SITE GQA RATE OF $16.7 \%$ IS APPLIED TO ALL COSTS TO LTQUTDATE THE HANFORD GENERAL \& ADMINISTRATIVE COSTS.

(3) HANFORD SITE MPR RATE OF $7.0 \%$ IS APPLIED TO ALL PURCHASED MATERIAL ANO $7.7 \%$ TO ALL PURCHASED SERICES TO LEQUIDRTE THE COST OF PROCUREMENT (INCLUDING RECIEVING).

FDNW APPLIES THE ABOVE FACTORS TO ESTIMATEO COSTS AS FOLLOWS:

(1) FOH GFS/G\&A CM FACIOR: A COMPOSITE FACTOR OF $33.04 \%$ HAS BEEN APPLIEO TO TOIAL FDNH FIXED PRICE CONSTRUCTION

(2) MANAGEHENT HHICH INCLUDES GOVERNHENT FURNISHED SERVICES (GFS) AND SITE G\&A/FEE.

(2) FDH GFS/G\&A LABOR FACTOR: A COMPOSITE FACTOR HAS BEEN APPLIED TO TOTAL FDNH LABOR COSTS AS FOLLOUS AE/CM COSTS $=33.04 \%$, FDNWS CONSTRUCTION LABOR $=28.37 \%$, FDNWS CONSTRUCTION MANAGEMENT LABOR = 33 . OL\%, FDNW CONTRACT

(3) FOH MPR/G\&A HATERIAL FACTOR: A COMPOSITE FACTOR OF $24.87 \%$ HAS BEEN APPLJED TO TOTAL FONH MATERIAL COST HHICH INCLUDE A MPR OF $7 \%$ AND MATERIAL GQA /FEE OF $16.7 \%$

4. ESCALATION

ESCALATION PERCEUTAGES WERE CALCULATED FROM THE JANUARY 1997 UPDATE OF IHE ECONOMIC ESCALATION PRICE CHAHGE INOICES FOR DOE CONSTRUCTION PROJECTS AS PUBLISHED BY THE HOFFICE OF INFRASTRUCTURE ACQUISITION"FM-50.

5. CONTINGENCY

A. DEFINITION OF COHIINGENCY AS PROVIDED BY DOE

"CONTINGENCY COVERS COSTS THAT MAY RESULT FROM INCOMPLETE OESIGN, UNFORESEEN AND UNPREDICTABLE CONDITIONS, OR

UNCERTAINTIES HITHIH THE DEFINED PROJECT SCOPE. THE AMOUNT OF CONIIHGENCY HILL DEPEND ON THE STATUS OF. DESIGH,

PROCUREMENT, AND CONSTRUCTION; AND THE COMPLEXITY AND UNCERTAINTIES OF THE COMPONENT PARTS OF THE PROJECT. CONTINGENCY IS NOT TO BE USED TO AVOIO MAKING AN ACCURATE ASSESSMENT OF EXPECTED COSTU (OFFICE OF WASTE MANAGEMENT (EEH-ZO) COST AND SCHEDULE GUIDE.

B. COntThgency allowance gutdelihes

THE DOE GUIDELINE CONTINGENCY ALLONANCE FOR A PLANIING/FEASIBILITY ESTIMATE IS $20 \%$ to $30 \%$.

C. METHODOLOGY

CONTINGENCY IS EVALUATEO AT THE LOWEST WORK BREAKOOWN STRUCTURE (WBS) LEVEL WITHIN THE COST ESTIMATE DETAILS. IT IS SUMHARIZED AT UPPER HBS LEVELS AND REPORTED ON THE SUMMARY REPORTS.

D. ANALYSIS

AN ASSESSMENT OF DESIGN MATURITY, WORK COMPLEXITY AND PROJECT UNCERTAINTIES HAS BEEM PERFORMED. AN EXPLAINATION OF THIS ASSESSMENT AND CONTIGENCY RATES WHICH HAVE BEEN ADDED TO THE COST OF WORK ARE AS FOLLOWS

HBS $31 X X X X$ CONSTRUCTION, ENGIHEERIHG AND PROJECT MANAGEMENT - A $30 \%$ CONTINGENCY HAS BEEN APPLIED TO COVER COSTS LIKELY TO SURFACE THAT HAVE NOT BEEN ANTICIPATED AT THIS EARLY STAGE OF PLANHING. TECHMIQUES TO BE UTILIZED FOR DECONTAMIHATION, MANY OF THE ACTIVITIES WILI BE SUBJECT TO CHANOE AT ANY TIME DURING PROGRESS. 
FLUOR DANIEL NORTHHEST, INC. LOCKHEED MARTIN HAHFORD CORP. JOB NO. E61945/F3RULS

FILE NO. 2475AABI

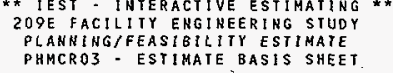

PAGE 5 OF 9

DATE $06 / 16 / 97 \quad 14: 17: 07$

BY DXH/RWO

6. REMARKS

MAJOR ASSUMPTIONS WHICH haVE BEEN MADE IN THE PREPARATION OF THIS ESITMATE ARE AS FOLLOUS:

A.) COSTS FOR CLOSURE CERTIFICATIONS, WIPE SAMPLES, SAMPLE ANALYTICAL COSTS; CORE SAMPLES, SOIL SAMPLES TECHMICAL PUBLICATIONS, VERIFICATION SAMPLING AND A DECOMMISIONING PLAN HAVE NOT BEEN ADDRESSED, AND IF
THESE ARE REQUIRED, WILL HEED TO BE INCLUDED.

B.) LIMITED INFORMATION WAS AVAILABLE AND GROSS ASSUMPTIONS WERE MADE.

NOT BE ENCOUNTERED.

AS A PERCEMTAGE OF CONSTRUCTION, SEE R08 REPORT. 
FLUOR DAHIEL HORTHUEST, INC.

LOCXHEED MARTIN HAHFORD CORP.

JOB NO. EOT945/F3RUL

FILE HO. 2475AAB 1

SORT

CODE/WBS

DESCRIPTION

CORT

FDNH FLUOR DANIEL HORTHHEST

310100 ALTERHATIVE 1 - LEAVE AS IS TOTAL GDHW FLUOR DANIEL NORTHWEST
** IEST - INTERACTIVE ESTIMATING *

209E FACILITY ENGINEERING STUDY PLANNIHG/FEASIBILITY ESTIMATE - ALTERNATIVE PHMCRO4. COHPANY/WBS SUMMARY
PAGE 6 OF 9

DATE $06 / 17 / 97 \quad 13: 04: 10$

BY DKH/RHO

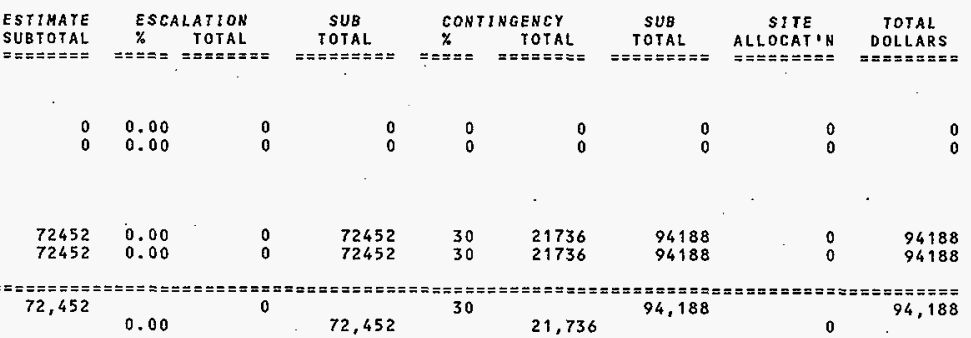

72,45

0.00

72,452

交 PROJECT TOTAL

100 ALTERUATIVE 1 - LEAVE AS IS

TOTAL LMHC LOCKHEED MARTIN HANFORD COR

1.736 
FLUOR DANIEL NORTHWEST, IHC.

LOCKHEED MARTIN HANFORD CORP.

JOB NO. E61945/F3RULS

FILE HO. Z475AAB ?

WBS DESCRIPTION

WBS

310100 alternative 1 - LEAVE AS IS

PROJECT TOTAL
** IEST - INTERACTIVE ESTIMATING * *

209E FACILITY ENGINEERING STUDY

PHACROS - CONSTRUCTION MANAGEMENT/OTHER COST SUMHARY
PAGE 7 OF 9

DATE 06/17/97 13:04:13

\begin{tabular}{|c|c|c|c|c|c|}
\hline $\begin{array}{l}\text { ESTIMATE } \\
\text { SUB TOTAL } \\
=======\end{array}$ & $\begin{array}{c}\text { CONSTRUCTION } \\
=== \pm==\end{array}$ & $\begin{array}{l}\text { N RANAGEMENT } \\
\text { TOTAL } \\
========\end{array}$ & $\begin{array}{c}\text { OTHER } \\
\text { COSTS } \\
=== \pm===\end{array}$ & $\begin{array}{c}\text { SUB } \\
\text { TOTAL } \\
=======\end{array}$ & 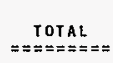 \\
\hline 72452 & 0.00 & 0 & 0 & 0 & 72452 \\
\hline
\end{tabular}

72,452

0
72,452 


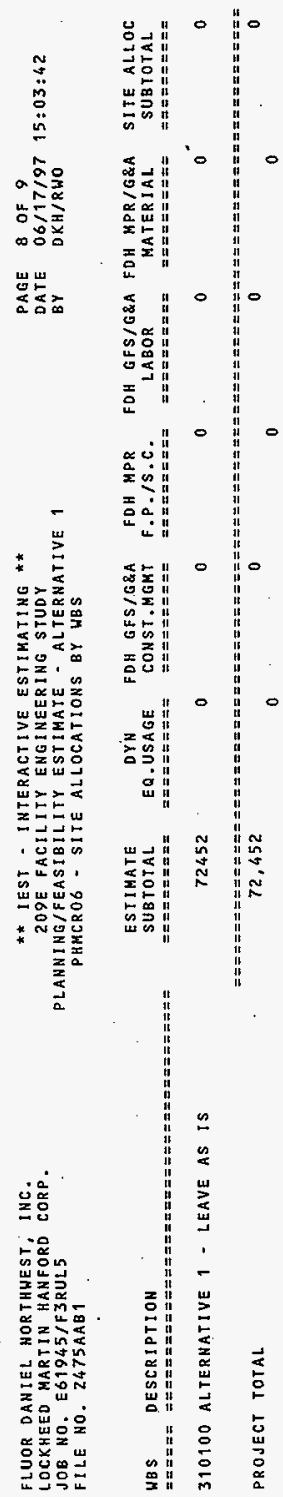


FLUOR DAHIEL NORTHWEST, INC. LOCKHEED MARTIN HANFORD CORP. JOB NO. EO1945/F3RUL FILE NO. 2475 AAB 1 .

HBS DESCRIPTION

WBS DOSCRIPTION

310100 ALTERNATIVE 1 - LEAVE AS IS

PROJECT TOTAL
* * IEST - INTERACTIVE ESTIMATING **

209E FACILITY ENGINEERIHG STUDY
PLANHING PHMCRO7. SITE ALLOCATION ESCALATION/CONTINGENCY REPORT
PAGE 8 OF 9

DATE 06/17/97 13:04:16 
FLUOR DANIEL NORTHHEST, INC. LOCKHEED MARTIN HANFORD CORP. JOB NO. E61945/F3RULS

FILE NO. 2475AAB 1

ACCOUNT

HUABER DESCRIPTIOH
* IEST - INTERACTIVE ESTIMATIMG * 209E FACILITY ENGINEERING STUDY PLANNIHG/FEASIBILITY ESTIMRTE - ALTERNATIVE PHMCROS. ESTIMATE DETAIL BY WBS $/$ COST CODE
PAGE 0 OA $06 / 17 / 97 \quad 13: 04: 19$ BY OXH/RWO

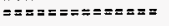

310100

al. TERHATIVE 1 - Leave as IS

310100.00

$310100.0001000 * * * * * * * * * * * * * * * * * * * * * * * * *$ DEFINITIVE DESIGN, ENGINEERIHG / INSPECTION, PROJ. HGMT.

SUB- EQUIP. OH\&P TOTAL. CODE QUAHTITY MANHOURS LABOR USAGE MATERIAL COHTRACT MENT I B \& DOLLARS 310100.0001001 THERE ARE NO COSTS FOR ANY

SUBTOTAL TECHNICAL SERVICES

TOYAL COST CODE. O0000

WBS 310100

CESCALATION $0.00 \%$ - CONTINGENCY $30.00 \%$

000

o

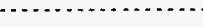

o

o

SITENORK

310100.02

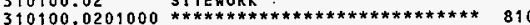
CONTINUE CURRENT SURVEIL-

LANCE AND MAINTENANCE

$\hbar * \hbar * \star * \star * * * * * * * \pi$

310100.0201002 SURVEILLANCE AND MAINTENANCE 810 $209-E)$

310100.0201004 SURYEILLANCE ANO MAINTENANCE 810

310100.0201012 HEALTH PHYS1CS SURVEILLANGE 810 ACTIVITIES AT 4 HR/WK

310100.0201020 POHER, HVAC, WATER AND
POEHTE LIGHTING FOR ONE YEAR YEAR***

310100.0201022 SANITARY WATER AHD SEWER

310100,0201024 ELECTRICAL UTILITY

MAINTENANCE

MAINIENANCE
BPA ELECTRICITY

SUBTOTAL, SITEWORK

TOTAL COST CODE 81002 WBS 310100

\begin{tabular}{|c|c|c|c|c|c|c|c|c|c|}
\hline 810 & $1 \mathrm{YR}$ & 0 & 0 & 0 & 0 & 3000 & 0 & 0 & 3000 \\
\hline 810 & $1 \mathrm{YR}$ & 84 & 4014 & 0 & 0 & 0 & 0 & 0 & 4014 \\
\hline 810 & $1 \mathrm{YR}$ & 0 & 0 & 0 & 0 & 10000 & 0 & 0 & 10000 \\
\hline & & & 59,452 & 0 & 0 & 13,000 & 0 & 0 & 72,452 \\
\hline & & & 59,452 & 0 & 0 & 13,000 & 0 & 0 & 72,452 \\
\hline
\end{tabular}


FLUOR DANIEL NORTHHEST, INC.

LOCKHEED MARTIN HANFORD CORP

JOB HO. E61945/F3RULS

FILE NO. 2475AAB 1

ACCOUNT

HUMBER

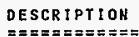

(ESCALATION

$0.00 \%$ - CONTINGENCY

** IEST - INTERACTIVE ESTIHATING * * 209E FACILITY ENGINEERING STUDY PLANNING/FEASIBILITY ESTIMATE - ALTERNATIVE
PHACROB - ESTIMATE OETAIL BY HBS / COST CODE

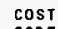

$\cos T$

COST QUANTITY MANHOURS LABOR UQUIP
PAGE DATE $06 / 17 / 97 \quad 13: 04: 19$

BY DKH/RWD

QUIP SUB -

SUB -
CNTRACT

EQUIP - OH\&P TOIAL

MENT $I$ B\& \& DOLLARS

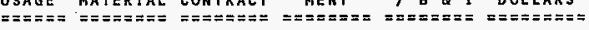

TOTAL hBS 310100 ALtERnATIVE 1 - LEAVE AS IS

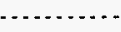

$\therefore, 202$

59,452

0

0

13,000

0

.

72,452 
FLUOR DAHIEL NORTHWEST, INC.

LOCKHEED MARTIH HANFORD CORP.

JOB NO. EGT945/F3RULS

FILE NO. Z475AAB T

ACCOUNT

NUMBER

DESCRIPTION
* IEST - INTERACTIVE ESTIMATING *

2.09E FACILITY ENGIHEERIWG STUDY

PLANNING/FEASIBILITY ESTIMATE - ALTERHATIVE

PHACROB- ESTIMATE DETAIL BY HBS / COST CODE

$\cos T$

COST QUANTITY MANHOURS. LABOR EQUIP

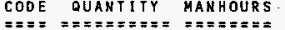

MATERIAL CONTRAC

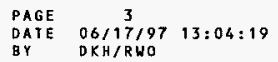

EQUIP- OH\&P TOTAL MENT 18 \& I DOLLARS

REPORT TOIAL

1,202

59,452

13,000

72,452 
FLUOR DANIEL NORTHHEST, INC.

LOCKHEED MARTIN HANFORO CORP.

JOB NO. EGTQRSTFBRULS

$209 E$ FACILIIY ENGINEERI IHG STUDY

PLANNING/FEASIBILITY ESTIMATE. RLTERMATIVE 2

PHMCRO1. PROJECY COST SUMMARY
PAGE 1 OF 9

$13: 23: 34$

DKH 18 NO

\begin{tabular}{|c|c|}
\hline $\begin{array}{l}\text { SORT } \\
=== \pm==\end{array}$ & 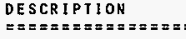 \\
\hline FDNH & FLUOR DANIEL NOR \\
\hline LMHC & LOCKHEEO MARTIN \\
\hline & TOTAL \\
\hline SITE & SITE ALLOCATIONS \\
\hline
\end{tabular}

\begin{tabular}{|c|c|c|c|}
\hline $\begin{array}{l}\text { ESCALATED } \\
\text { TOTAL COST } \\
=========\end{array}$ & $=x^{\operatorname{CON}}$ & 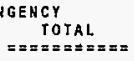 & $\begin{array}{c}\text { TOTAL } \\
\text { DOLLARS } \\
=====\approx===\end{array}$ \\
\hline 342,006 & 30 & 102,602 & 444,608 \\
\hline 99,804 & 30 & 29,941 & 129,745 \\
\hline
\end{tabular}

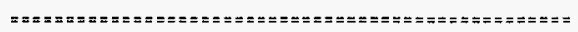

$\begin{array}{cccr}441,810 & 30 & 132,543 & 574,353 \\ 119,316 & 30 & 35,795 & 155,111 \\ 561,126 & 30 & 168,338 & 729,464\end{array}$

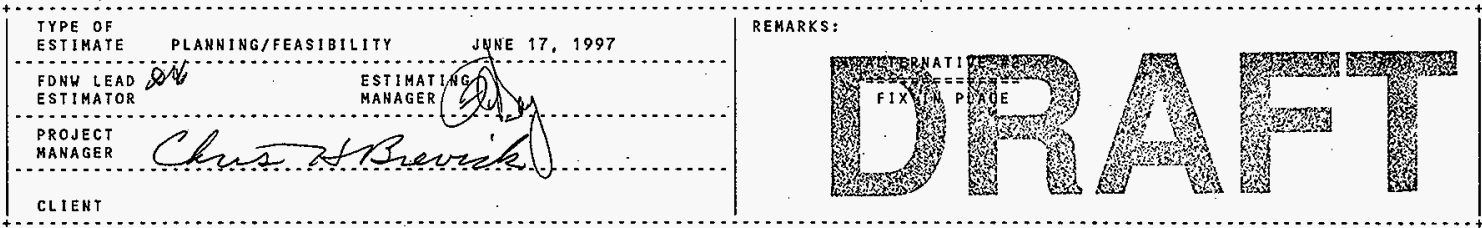


FLUOR DANIEL NORTHUEST, INC. LOCKHEED MARTIH KANFORD CORP.

FIIE 10.2475 AAC1

HBS DESCRIPTION

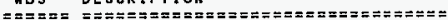

310200 ALTERHATIVE 2 - FIX IN PLACE

PROJECT TOTAL
* IEST - INTERACTIVE ESTIMATING ** 209E FACIIITY ENG INEER ING SIUDY

PLANHING FEASIBIL ITY ESTIMATE - ALTERNATIVE PHMCROZ - WORK BREAKDOWN STRUCTURE (HBS) SUMMARY
PAGE 2 OF 9

DATE $06 / 17 / 97 \quad 13: 23: 37$

\begin{tabular}{|c|c|c|c|c|c|c|c|c|}
\hline $\begin{array}{l}\text { ESTINATE } \\
\text { SUBTOTAL } \\
===\pi===\end{array}$ & $\begin{aligned} & \operatorname{ESCA} \\
& \not \\
&== \pm=\end{aligned}$ & $\begin{array}{l}\text { ALATION } \\
\text { TOTAL } \\
=======\end{array}$ & $\begin{array}{c}\text { SUB } \\
\text { TOIAL } \\
========\end{array}$ & $\begin{aligned} & \operatorname{CONT} \\
& \% \\
&===\end{aligned}$ & $\begin{array}{l}\text { INGEHCY } \\
\text { TOTAL } \\
=======\end{array}$ & $\begin{array}{c}\text { SUB } \\
\text { TOIAL. } \\
======\pi=\end{array}$ & $\begin{array}{c}\text { SITE } \\
\text { ALLOCAT'N } \\
========\end{array}$ & $\begin{array}{c}\text { TOTAL } \\
\text { DOLLARS } \\
=======\end{array}$ \\
\hline $44 t 810$ & 0.00 & 0 & 441810 & 30 & 132543 & 574353 & 155111 & 729464 \\
\hline
\end{tabular}


FLUOR DANIEL NORTHWEST, INC. LOCKHEED MARTIH HANFORD CORP. J08 NO. E61945/F3RULS

FILE NO. Z475AAC

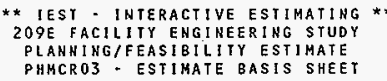

PAGE 3 OF 9

$14: 17: 07$

BY DKH/RHO

1. ESTIMATE PURPOSE

THIS ESTIMATE HILL BE USED FOR A SCOPING STUDY.

2. ESIIMAIE TECHNICAL BASIS

A. THIS ESIIMATE HAS BEEN PREPARED FOR LOCKHEEO MARYIN HANFORD CORP. AS REQUESTED BY FDNH PROJECT MANAGEMENT.

B. A ROUGH DESCRIPTION OF THE SCOPE OF WORK HAY BE FOUND IN THE OUTLINE OF ALTERNAIIVES, AN UHDATED ALIO

UNNUABERED DOCUMENT.

C. THE FOLLONING CONSTRAINTS AND/OR SPECIAL CONOITIONS EXIST: ALL WORK DOHE IN THE CRITICAL ASSEMBLY ROOM AND IR THE MIXING ROOH WILL REOUIRE FOLLOHING THE APPROPRIAIE RADIAIION WORK PROCEDURE.

3. ESTIMATE METHODOLOGY

A. DIRECT COSTS:

A MAMLORDING TECHALQUE WAS USEO TO COME UP HITH THE LABOR HOURS WHEN NECESSARY DUE TO THE UNDEFINED SCOPE.

(1) CONSTRUCTION LABOR, MATERIAL AND EQUIPMENI UHISS HAVE BEEN ESTIMATED BASED UPON ONE OR MORE OF YHE FOLLOHING STARDARD COMMERCIAL ESTIMATING RESOURCES, PUBLISHED ESTIMATING MANUALS AND R.S. MEANS

B. DIRECT COST FACTORS

(1) SALES TAX HAS BEEN APPLIED TO ALL MATERIALS AND EQUIPMENT PURCHASES AT 8\%.

(1)

(3) AN ESTIMATING FACTOR OF $15 \%$ HAS BEEN APPLIED TO DIRECT CRAFT LABOR FOR GENERAL REQUIREMENTS AND $23.58 \%$ FOR TECHUICAL SERVICES.

(4) CONSUMABLES ARE ESTIMATED AT 3 . $2 \%$ OF DIRECT CRAFT LABOR COSTS

(5) SPECIALLESARE ESTIMATED AT S. $\%$ OF DIRECT CRAFT LABORT OSIS.T LABOR FOR ACTUAL TIME LOST OUE TO THE PERSOUNEL PROTECT IVE EQUIPMENT AND PROCEDURES. THE RATES HHICH HAVE BEEN APPLIED ARE AS FOLLOWS:

PROTECTIVE CLOTHING FACTOR HAS BEEN APPLIED AT $15 \%$

MASK HORK AT 85\% PLUS $15 \%$ FOR PROTECTIVE. CLOTHING. BELIEVE TO BE A CONCERN FOR THESE ACTIVITIES.

(6) PREMIUM PAY

OVERTIME REOUIREMENTS AND SHIFT DIFFERENTIAL PAY FOR CRAFT LABOR HILL NOT BE REQUIRED FOR THIS WORK, AS STRAIGHT IIME, 40 HOUR HEEKS ARE DEEMED ADEQUATE TO ACCOHPLISH THIS WORK.

(7) GENERAL FOREMAN FACTOR OF 7\% HAS BEEN APPLIED TO DIRECT CRAFT LABOR CREUS.

(7) GEAERAL HOREMAN FACTOR OF T\% HAS BEEN

GOVERNMENT OWNED EQUIPMENT CONTROLLED BY DYNCORP

C. RATES

(1) FLUOR DANIEL NORTHWEST LABOR RATES ARE 8ASED UPON THE FLUOR DAINEL FEDERAL OPERATIONS (FEDFO) DISCLOSURE STATEMENT AND APPROVED PROVISIONAL BILLING RATES. FOR ESTIMATING PURPOSES, AVERAGE RATES BY OPERATIOHS CODE HAVE BEER DEVELOPED BASED UPON RECENT COST HISTORY:

(2) FLUOR DANIEL NORTHHEST SERVICES (CONSTRUCTION CRAFT LABOR) RATES ARE THOSE LISTED IN APPENDIX A TO THE HAHFORD SITE STABILIZATION AGREERENT.

(3) FDH \& PHMC SUBCONTRACTOR STANDARD LABOR RATES ARE THOSE.LISTED IN THE FINAHCIAL DATA SYSTEM (FDS) FDST $321 R$ REPORT ORGANIZATIOR RATES PLUS ADDERS. 
FLUOR DANIEL MORTHWEST, INC. LOCKHEED MARTIN HANFORD CORP. JOB HO. E61945/F3RULS

FILE NO. 2475AACI

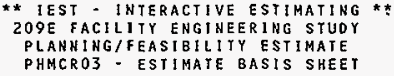

PAGE 4 OF 9

DATE $06 / 16 / 97 \quad 14: 17: 07$

BY DKH/RWO

D. STIE ALLOCATIONS FACTORS

SITE ALLOCATION FACTORS ARE DEVELOPED AND PROVIDED BY FLUOR DAMIEL HANFORD (FOH) FOR ESTIMATING USE.

(1) GOVERNAENT FURMISHED SERVICES RATE IS APPLIED TO ALL COSTS IO LIQUIDATE GOVERHMENT FURNISHED SERVICES PROVIDED TO THE ENTERPRISE COMPANIES: $14 \%$ FOR FDNH, $10 \%$ FOR FDNWS (CONSTRUCTION).

(2) HANFORD SITE G\&A RATE OF 16.7\% IS APPLIED TO ALL COSTS TO LIOUTDATE THE HANFORD GENERAL \& ADMINISTRATIVE COSTS.

(3) HANFORD SITE MPR RATE OF $7.0 \%$ 1S APPLIED TO ALL PURCHASED MATERIAL AND $7.7 \%$ TO ALL PURCHASED SERICES TO LIQUIDATE THE COST OF PROCUREMENT (IHCLUDING RECIEVING).

FONW APPLIES THE ABOVE FACTORS TO ESTIMATED COSTS AS FOLLOWS:

(1) FDH GFS /G\&A CH FACTOR: A COMPOSITE FACTOR OF 33.04\% HAS BEEN APPLIED TO TOTAL FDNW FIXED PRICE CONSTRUCTION

(2) MANAGEMENT WHICR INCLUDES GOVERNHENT FURHISHED SERVICES (GFS) AND SITE G\&A/FEE.

(2) FDH GFS/G\&A LABOR FACTOR: A COMPOSITE FACTOR HAS BEEN APPLIED TO TOIAL FDHW LABOR COSTS AS FOLLOWS AE/CM COSTS $=33.04 \%$, FDNUS CONSTRUCTION LABOR $=28.37 \%$, FDNHS CONSTRUCTION MANAGEMENT LABOR = $33.04 \%$, FDHW CONTRACT

(3) FOH MPR/GQA MATERIAL FACTOR: A COMPOSIIE FACTOR OF $24.87 \%$ HAS QEEN APPLIED TO TOIAL FONH MATERIAL COST HHICH INCLUDE WOH MPR OFG $7 \%$ MATER MATERIAL GRA/FEE OF $16.7 \%$

4. ESCALATION

ESCALATION PERCENTAGES HERE CALCULATEO FROM THE JANUARY 1997 UPDATE OF THE ECONOMIC ESCALATION PRICE CHANGE INDICES FOR DOE CONSTRUCTION PROJECTS AS PUBLISHEO BY THE "OFFICE OF INFRASTRUCTURE ACQUISITIONH FM.SO.

5. CONTINGENCY

A. DEFINITION OF CONTINGENCY AS PROVIDED BY DOE

"CONTINGENCY COVERS. COSTS THAT MAY RESULT FROH IHCOMPLETE DESIGN, UNFORESEEN AND UNPREDICTABLE CONDITIONS, OR UNCERTAINTIES WITHIN THE DEFINED PROJECT SCOPE. THE AMOUHT OF CONTINGENCY WILL DEPEND OH THE STATUS OF DESIGN PROCUREMENT, AND CONSTRUCTION: AND THE COMPLEXITY AND UNCERTAINIIES OF THE COMPONENT PARTS OF THE PROJECT. CONIINGENCY IS HOT TO BE USED TO AVOID MAKING AN ACCURATE ASSESSMENT OF EXPECTED COST" COFFICE OF HASTE MANAGEMENT (ER-3O) COST ARO SGHEDULE GUIDE.

B. CONIINGEMCY ALLOHANCE gUIDELINES

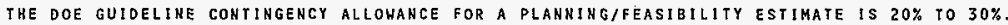

C. METHODOLOGY

CONIINGENCY IS EVALUATED AT THE LOHEST WORK BREAKDOWN STRUCTURE (WBS) LEVEL WITHIN THE COST.ESTIMATE DETAILS. IT IS SUMMARIZED AT UPPER HBS LEVELS AND REPORTED ON THE SUMMARY REPORYS

D. ANALYSIS

AN ASSESSHENT OF DESIGN MATURITY, WORK COMPLEXITY ANO PROJECT UNCERTAINTIES HAS BEEN PERFORMED. AN EXPLAIMATION OF THIS ASSESSHENT SND CONTIGENCY RATES WHICH HAVE BEEN ADDED TO THE COST OF WORK ARE AS FOLLOWS:

WBS 31 XXXX CONSTRUCTION, ENGINEERING AND PROJECT MANAGEMENT - A $30 \%$ CONTIMGENCY HAS BEEN APPLIED TO COVER COSTS LIXELY TO SURFACE THAT HAVE NOT BEEN ANTICIPATED AT THIS EARLY SIAGE OF PLANHING. TECHNIOUES TO BE UTILIZED FOR DECONTAMINATION, STABILIZATION AND REMOVAL WILL NEED FURTHER STUDY AND MALY 
FLUOR DANIEL NORTHWEST, INC. LOCKHEED MARTIN HANFORD CORP. JOB NO. E6194.5/F3RULS

FILE NO. 2475 AACI

\author{
* * IEST - INTERACIIVE ESTIHATING ** \\ 209E FACILITY ENGINEERIHG STUDY \\ PLANMING/FEASIBILITY ESTIMAIE \\ PHMCROZ - ESTIMATE BASIS SHEET
}

PAGE 5 OF 9

DATE O6/16/97 $14: 17: 07$

\section{REMARKS}

HAJOR ASSUMPTIONS WHICH HAVE BEEN MADE IN THE PREPARATION OF THIS ESITMATE ARE AS FOLLOWS:

A.) COSTS FOR CLOSURE CERTIFICATIONS, HIPE SAMPLES, SAMPLE ANALYTICAL COSIS, CORE SAMPLES, SOIL SAMPLES, TECHNI CAL PUBLICATIONS, VERIFICATION SAMPLING AND A DECOMMISIONING PLAN HAVE NOT BEEN ADDRESSEO, ANO IF
THESE ARE REQUIRED, HILL NEED TO BE INCLUDED.

B. ) LIMITED INFORMATION WAS AVAILABLE ANO GROSS ASSUMPIIONS HERE MADE.

D.) Enginering and PROJECt maragement cosis. Were figured as a percentage of construction, see rob report. 
FLUOR DANIEL NORTHWEST, INC.

LOCKHEED HARTIN HAHFORD CORP

JOB NO. E61945/F3RULS

SOR I

CODE/WBS DESCRIPTION

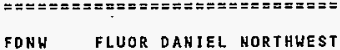

310200 ALTERHATIVE 2 - FIX IN PLACE TOTAL FONW FLUOR DANIEL NORTHWEST
* * IEST - INTERACTIVE ESTIMATIHG

209E FACILITY ENG INEERING STUDY

PLANHING/FEASIBILITY ESTIMATE - ALTERNATIVE

PHMCRO4 - COMPANY/HBS SUMHARY $\begin{array}{lll}\text { PAGE } & 6 & \text { OF } 9 \\ \text { DATE } & 06 / 17 / 97 & 13: 23: 40\end{array}$

BY DKH/RWO

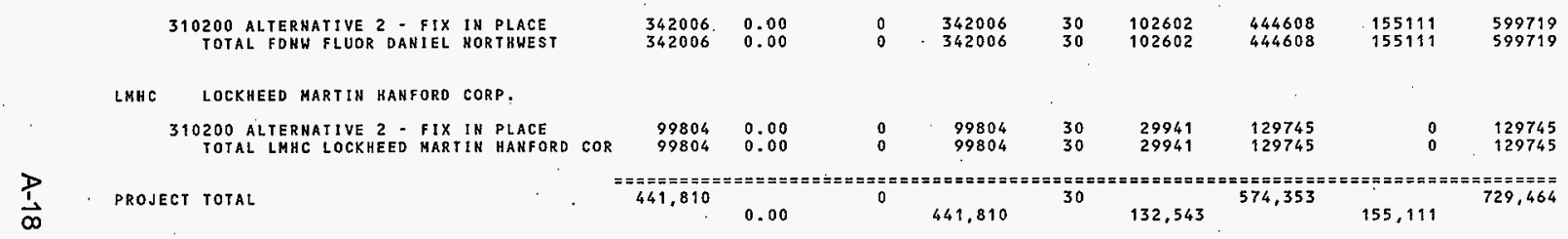


FLUOR DAHIEL NORTHHEST, INC. LOCKHEED MARTIN HANFORD CORP. JOB NO. E61945/F3RUL5

FILE NO. Z475AAC

WBS DESCRIPIION
WST

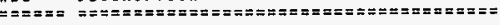

310200 alternative 2 - FIX IN PLACE

PROJECT TOTAL
** IEST - INTERACTIVE ESTIMATING *

209E FACILITY ENGINEERING STUDY

PLANNIHG/FEASIBILITY ESTIMATE - ALTERNATIVE 2 PHMCROG - SITE ALLOCATIONS BY WBS
PAGE 8 OF 9

DATE 06/17/97 15:04:17

BY DKH/RHO

\begin{tabular}{|c|c|c|c|c|c|c|}
\hline $\begin{array}{l}\text { ESTIMATE } \\
\text { SUB TOTAL } \\
=========\end{array}$ & $\begin{array}{c}\text { DYN } \\
\text { EQ. USAGE } \\
========2\end{array}$ & $\begin{array}{l}\text { FDH GFS/G\&A } \\
\text { CONST. MGMT } \\
========\Rightarrow\end{array}$ & $\begin{array}{l}F D H \quad M P R \\
F, P . / S \cdot C . \\
======m=2=\end{array}$ & $\begin{array}{c}\text { FDH GFS/G\&A } \\
\text { LABOR } \\
=========\end{array}$ & $\begin{array}{c}\text { FDH MPR/G\&A } \\
\text { MATER IAL } \\
=========8\end{array}$ & $\begin{array}{l}\text { SITE ALLOC } \\
\text { SUB TOTAL } \\
========\end{array}$ \\
\hline 403837 & 14518 & 12546 & o & 87420 & 4832 & 119316 \\
\hline
\end{tabular}

(1)

$\begin{array}{lllllll}403,837 & 14,518 & 12,546 & 87,420 & 119,316\end{array}$ 
FLUOR DANIEL HORTHWEST, INC. LOCKHEED MARTIN HANFORD CORP. JOB NO, E61945/F3RULS

FILE HO. Z475AAC1

HBS DESCRIPTTOH

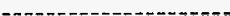

310200 alternative 2 - FIX In PLACE

PROJECT TOTAL
* * IEST - INTERACTIVE ESTIMATIMg * PLAHHING/FEASIBILITY ESTIMATE - ALTERHATIVE 2
PHMCRO7 - SITE ALLOCATION ESCALATIOH/CONIMGENCY REPORT
PAGE 9 OF 9

$13: 23: 45$

BY OKH/RWO

\begin{tabular}{|c|c|c|c|c|c|}
\hline $\begin{array}{l}\text { SITE ALLOC } \\
\text { SUBTOTAL. } \\
=========\end{array}$ & $\begin{array}{l}\text { ESCALATION } \\
\% \text { TOTAL } \\
===========\end{array}$ & $\begin{array}{c}\text { SUB } \\
\text { TOTAL } \\
== \pm== \pm==\end{array}$ & $=\underset{\%}{\operatorname{con} T}$ & $\begin{array}{l}\text { I NGENCY } \\
\text { IOYAL } \\
========\end{array}$ & $\begin{array}{c}\text { TOTAL } \\
\text { DOLLARS } \\
========\end{array}$ \\
\hline 119316 & 0.00 & 119316 & 30 & 35795 & 155111 \\
\hline $\begin{aligned}== \pm= & ====== \\
& 119,316\end{aligned}$ & 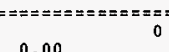 & $==\pi=\pi=\pi=$ & $===\frac{=}{30}$ & $========$ & $\begin{aligned} &==== \pm= \\
& 155,111\end{aligned}$ \\
\hline
\end{tabular}


FLUOR DANIEL MORTHUEST, INC.

LOCKHEED MARTIN HAHFORÓ CORP.

JOB NO. E6 \$945/F3RULS

FILE HO. 2475AAC1

ACCOUHT
HUMBER
$=====================$

alternative 2 - FIX IN PLACE

310200

IECHNICAL SERVICES

310200.00

310200.0002100 DEFINITIVE DESIGI

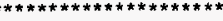

AT $25 \%$ OF CONSTRUCTION

000

ENGINEERING/ INSPECYION

AT $15 \%$ OF CONSTRUCTION

$310.200 .0002300 * * * \hbar * * * * * * * * * * * * * * * * * * * * * * * * 000$

PROJECT MANAGEMEHT

AT $15 \%$ OF CONSTRUCTION

SUBTOTAL TECHNICAL SERVICES

TOTAL

COST CODE 00000

HBS 310200

SESCALATION

310200.01 GENERAL REQUIRMENTS

310200.0102000 HPT TO MAH SIEP-OFF PAD

SUBTOTAL GENERAL REQUIRMENTS

SWP $100.00 \%$

TOTAL

COST CODE 81001

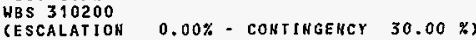

01

310200.02 SITEWORK

年 FIX IN PLACE ALL

* FOM TONTAMINATION

310200.0202102 APPLY FIXATIVE TO ALL 810 M $20000 \mathrm{SF}$ SURFACES 50 MIIL EPOXY.

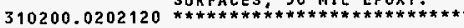
SEAL GLOVE BOX PORTS, EYC.
** IEST - INTERACTIVE ESTIHATING * 209E FACILITY ENGINEERING STUDY PHMCROB. ESTIMATE DETAIL BY WBS I COST CODE

EQUEP

EQUIP

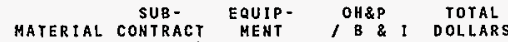

CODE QUANTITY MANHOURS LABOR USAGE MATERTAL CONTRACT MENT,

$\begin{array}{lll}\text { PAGE } & 1 \\ \text { DATE } & 06 / 17 / 97 & 13: 23: 48\end{array}$

\begin{tabular}{|c|c|c|c|c|c|c|c|c|c|}
\hline 000 & $1 \mathrm{~L} / \mathrm{S}$ & 985 & 65010 & 0 & 0 & 0 & 0 & 0 & 65010 \\
\hline 000 & $1 \mathrm{~L} / \mathrm{s}$ & 650 & 39000 & D & 0 & 0 & 0 & o & 39000 \\
\hline 000 & $1 \mathrm{~L} / \mathrm{s}$ & 534 & 38982 & 0 & 0 & 0 & 0 & 0 & 38982 \\
\hline & & .169 & 42,992 & 0 & 0 & 0 & 0 & 0 & 142,992 \\
\hline & & & 42,992 & & 0 & & 0 & & 142,992 \\
\hline
\end{tabular}



FLUOR DANIEL NORTHWEST, INC.
LOCKHEED MARTIN HANFORD CORP. JOB NO. E61945/F3RUL5

FILE NO. 2475AACI

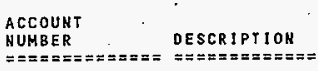

$* \pi * * * * * * * * * * * * * * * * * * * * * * * * * *$

310200.0202122 SEAL GLOVE BOX PORTS

INSTALL BLANK-OFFS WITH

310200.0202124 SEAL. HODD OPENIHGS

INSTALL CAPS AND SEAL OFF

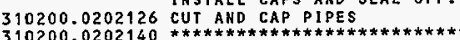

DISCONHECT VENTILATION

310200.0202142 DISCONKECT ACTIVE VENTIL

310200.0202142 DISCONNECT ACTIVE VENTIL.
ATIOH TO GLOVE BOXES/HOOD, ATION TO

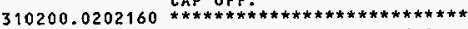
CONNECT PASSIVE HEPA

310200.0202162 CHECK PASSIVE HEPA FILTER BOXES/HOODS, SHOULD ALL BE EXISTING.

310200.0202180 Existing

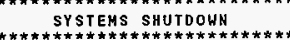

310200.0202182 SHUTDOHN ALL SYSTEMS WOT REQUIRED FOR SAFETY,

ALLOWANCE.

$310200,0202200 * * * * * * * * * * * * * * * * * * * * * * * * * * *$ POWER, HVAC, HATER AND

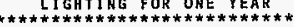

310200.0202204 ELECTRICAL UTILITY MAINIEMANCE

310200.0202206 BPA ELECTRICIYY, MINIHAL SUBTOTAL SITEHORK

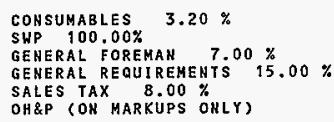

COST QUANTITY MANHOURS LABOR USAGE HATERIAL SUB. EQUTP- OH\&P TOTAL

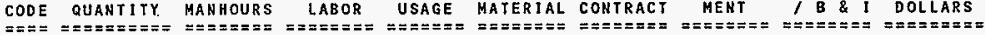

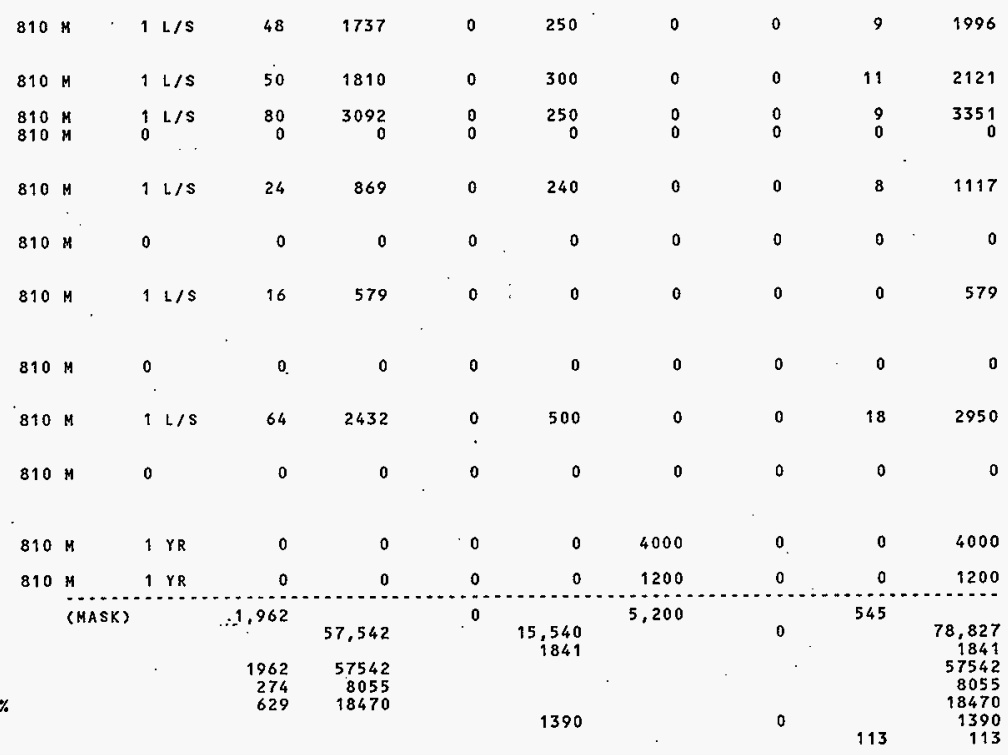


FLUOR DANIEL NORTHUEST, INC.
LOCKHEED MARTIN HANFORD CORP.

JO8 NO. E61945/F3RUL5

* IEST - INTERACTIVE ESTIMATING *

209E FACILITY ENGINEERING STUDY

FILE NO. Z475AAC1

PLANHIRG/FEASIBILIIY ESTIMATE - ALTERMAYIVE PHMCROB - ESTIMATE DETAIL BY WBS / COST CODE

\section{ACCOUNT \\ NUMBER \\ DESCRIPTION}

EQUiP

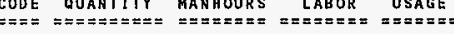

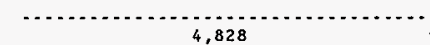

TOTAL

COST CODE 81002

HBS 310200
(ESCALATION

$0.00 \%$ - CONTINGEHCY

$30.00 \%$ SESCALATION
CODE OUANTITY MANHOURS LABOR USAGE

SUB -

MATERIAL CONTRACT

$18,771 \quad 5,200$

18,771

PAGE

DATE $\quad 06 / 17 / 97 \quad 13: 23: 48$

BY OKH/RWO

EQUIP- OH\&P TOTAL

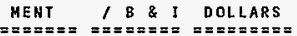

658

166,240

8,977

379,206

0

18,771

5,200

0

658

竔 
FLUOR DAHIEL NORTHUEST, INC.

JOB HO. E61945/F3RULL

ACCOUNT:

HUHBER

DESCRIPTION
LOCKHEED MART IN HAHFORD CORP.

FILE NO. 2475 AAC1

** IEST - INTERACTIVE ESTIMATING * 209E FACILITY ENGINEERING STUDY
PLANNIHG/FEASIBILITY ESTIMATE - ALTERNATIVE 2
PHMCROS - ESTIMATE DETALL BY HES/ COST CODE

\section{$\cos T$}

CODE QUAHTITY MANHOURS

EQQUTP

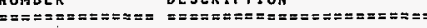

8,977

379,206
18,771
5,200

5,200 $\begin{array}{lll}\text { PAGE } & 4 & 4 \\ \text { DATE } & 06 / 17 / 97 & 13: 23: 48\end{array}$

BY DKH/RWO

REPORT TOIAL 
FLUOR DANIEL NORTHWEST, INC. LOCKHEED MARTIN HANFORD CORP. JOB HO. E61945/F3RUL5

* IEST - INTERACTIVE ESTIMATING * 209E FACILITY ENGINEERING STUDY PLANEING/FEASIBILITY ESTIMATE - ALTERNATIVE 3 PHMCROT. PROJECT COST SUMMARY
PAGE 1 OF 9

$13: 39: 29$

BY DKH/RHO

\begin{tabular}{|c|c|c|c|}
\hline $\begin{array}{l}\text { ESCALAYED } \\
\text { TOTAL COST } \\
==========\end{array}$ & $=\%^{\operatorname{CON}}$ & $\begin{array}{l}\text { NGENCY } \\
\text { IOTAL } \\
======\equiv===\end{array}$ & $\begin{array}{c}\text { TOIAL } \\
\text { OOLLARS } \\
===\Xi==\equiv===\end{array}$ \\
\hline 392,520 & 30 & 117,756 & 510,276 \\
\hline 264,194 & 30 & 79,258 & 343,452 \\
\hline 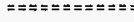 & 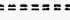 & 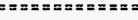 & 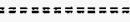 \\
\hline 656.714 & 30 & 197,014 & 853,728 \\
\hline 131,545 & 30 & 39,464 & 171,009 \\
\hline & & & $= \pm \equiv$ \\
\hline 788,259 & 30 & 236,478 & $1,024,737$ \\
\hline
\end{tabular}

PROJECT TOTAL

30

TYPE OF
ESTIMATE
$\begin{gathered}\text { FDNH IEAD } \\ \text { ESTIMATOR }\end{gathered}$
PROJECT
MANAGER
CLIENT

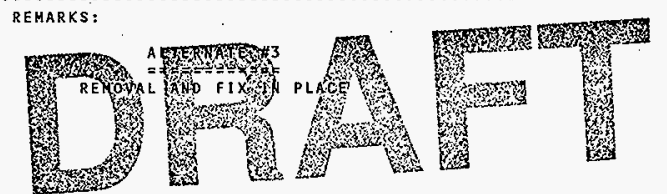


FLUOR DANIEL NORTHLEST, INC.

LOEKHEED MARTIN HAHFORD CORP

JOB HO. E61945/F3RULS

FILE HO. Z475AAD 1

HBS DESCRIPTION

Serion

310300 ALT. 3 - REMOVAL \& FIX IN PLACE

PROJECT TOIAL
** IEST - INTERACTIVE ESTIMATIHG *

OOQE FACILITY ENGINEERING STUDY

PLANNING/FEASIBILITY ESTIMATE - ALTERNATIVE 3 PHMCRO2 - WORK BREAKDOWN STRUCTURE (HBS) SUMMARY
PAGE 2 OF 9

$13: 39: 33$

BY DKH/RWO

\begin{tabular}{|c|c|c|c|c|c|c|c|c|}
\hline $\begin{array}{l}\text { EST I MATEE } \\
\text { SUB TOTAL } \\
========\end{array}$ & 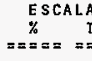 & $\begin{array}{l}\text { LATION } \\
\text { IOTAL } \\
==\pi==\pi=\end{array}$ & 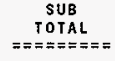 & $\begin{aligned} & \operatorname{CONT} \\
\% & ====\end{aligned}$ & $\begin{array}{l}\text { IHGENCY } \\
\text { IOTAL } \\
======\end{array}$ & $\begin{array}{c}\text { SUB } \\
\text { TOTAL. } \\
= \pm= \pm== \pm==\end{array}$ & $\begin{array}{c}\text { SITE } \\
\text { ALL OCAT N } \\
=========\end{array}$ & $\begin{array}{c}\text { TOTAL } \\
\text { DOLLARS } \\
========\end{array}$ \\
\hline 656714 & $0.00 \ldots$ & $\therefore$ & 656714 & 30 & 197014 & 853728 & 171009 & 1024737 \\
\hline
\end{tabular}

离 
FLUOR DANIEL HORTHWEST, INC.

LOCKHEED MARTIN HANFORD

FILE NO. 2475 AAD
* * IEST - INTERACTIVE ESTIMATING ** 209E FACILITY EHGINEERING STUD PLANNING/FEASIBILITY ESTINATE
PAGE 3 OF 9

DATE $, 06 / 16 / 97 \quad 14: 17: 07$

BY DKH $/$ RHO

1. ESTIMATE PURPOSE

THIS ESTIMATE WILL 8 E USED FOR A SCOPING STUDY.

2. ESTIMATE TECHNICAL BASIS

A. THIS ESTIMATE HAS BEEN PREPAREO FOR LOCKHEED MARTIN HANGORD CORP. AS REQUESTED BY FONH PROJECT MANAGEMENT.

A. THIS EST RATE HAS BEEN PREPAREO FO HORK KAY BE FOUND IN THE OUTLINE OF ALTERNATIVES, AN UNDATED AND

UNHUMBERED DOCUMENT

C. THE FOLLOWING CONSTRAINTS AND/OR SPECIAL CONDIIIONS EXIST: ALL WORK DONE IN THE CRITICAL ASSEMBLY ROOM AND IN THE MIXING ROOM WILL REQUIRE FOLLOHIMG THE APPROPRIATE RADIATION WORK PROCEDURE.

3. ESTIMATE METHODOLOGY

A. DIRECT COSTS:

TECHNIQUE HAS USED TO COME UP WITH THE LABOR HOURS NHEN NECESSARY DUE TO THE UNDEFINED SCOPE.

(1) CONSTRUCTIOH COMMERCIAL ESTIMATING RESOURCES, PUBLISHED ESIIHATIHG MANUALS AHD R.S. MEANS

8. DIRECT COST FACTORS

(1) SALES TAX HAS BEEN APPLIED TO ALL MATERIALS AND EQUIPMENT PURCHASES AT $8 \%$.

(IATS

(3) AN ESTIMATING FACTOR OF 15\% HAS

(4) 23.58\% FOR TECHATYOR SERVICES; $\%$ OF DIRECT CRAFT LABOR COSTS.

(5) CONUABLES ARE ESTMATED AT 3. PROTEL WE PROTECTIVE CLOTHING FACTOR HAS BEEN APPLIED AT $15 \%$ MASK HORK AT B5\% PLUS $15 \%$ FOR PROTECTIVE CLOIBING. CONTAHINATION RESTRTCTHOHS ASSOCHATED WITHISDI

(6) PREMIUH PAY PQERTIME REQUIREMEHTS AHD SHIFT DIFFEREMTIAL PAY FOR CRAFT LABOR HILI NOT BE REQUIRED FOR THIS WORK, AS STRAIGHT TIME 40 HOUR WEEKS ARE DEEMED ADEQUATE TO ACCOMPLISH IHIS HORK.

TENERAL FOREMAU GACTOR OF 7\% HAS BEEN APPLIED TO DIRECT CRAFT LABOR CREUS.

(8) A FACTOR OF IO\% HAS BEEN GOVERNMENT OHMED EQUIPMENT CONTROLLED BY DYNCORP.

C. RATES

(1) FLUOR DANIEL HORTHHEST LABOR RATES ARE BASED UPON THE FLUOR OATNEL FEOERAL OPERATIONS (FEDFO) DOSCLOSURE SEETE AND APPAOVED PRO UPON RECENT COST HISIORY.

(2) FLUOR DANIEL HORTHWEST SERVICES (CONSTRUCTION CRAFY LABOR) RATES ARE IHOSE LISTED IN APPENDIX A TO. THE HANFORD SITE STABILIZATIOU AGPEEMENT.

(3) FDH \& PHMC SUBCONTRACTOR STANDARD LABOR RATES ARE THOSE LISTED IN THE FIHAHCIAL DATA SYSTEM (FDS) FDSE $321 R$ REPORT DRGANIZATION RATES PLUS ADDERS. 
FLUOR DANIEL NORTHLEST, INC. LOCKHEED MARTIN HANFORD CORP. JOB NO. E69945/F3RUL5

FILE NO. Z475AAD?

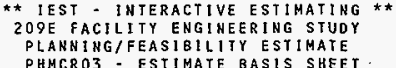

PAGE 4 OF 9

DATE $\quad 06 / 16 / 97 \quad 14: 17: 07$

BY OKH/RWO

D. Site ALLOCATIONS FACTORS

SITE ALLOCATION FACTORS ARE DEVELOPED AND PROVIDED BY FLUOR DANIEL HANFORD (FDH) FOR ESTIMATING USE.

(1) GOVERHMENT FURMISHED SERVICES RATE IS APPLIED TO ALL COSTS TO LIQUIDATE GOVERMHENT FURNISHED SERVICES PROVIDED TO THE ENTERPRISE COMPANIES: $14 \%$ FOR FDNW, $10 \%$ FOR FDHHS (CONSTRUCTION).

EF

(3) HANFORD SITE MPR RATE OF $7.0 \%$ IS APPLIED TO ALL PURCKASEO MATERIAL AND $7.7 \%$ TO ALL PURCHASEO SERICES IO LIQUIDATE THE COST OF PROCUREMENT (INCLUDING RECIEVING).

FDNW APPLIES THE ABOVE FACTORS TO ESTIMATED COSTS AS FOLLOHS:

(1) FDH GFS/G\&A CH FACTOR: A COMPOSITE FACTOR OF $33.04 \%$ HAS BEEN APPLIED TO TOTAL FDNH FIXED PRICE CONSTRUCTIOA

(2) MANAGEMEMT HHICH IMCLUDES GOVERMMENT FURIISHED SERVICES (GFS) AND SITE G\&A/FEE. AE/CM COSTS $=33.04 \%$, FDNWS CONSTRUCTION LABOR $=28.37 \%$, FONWS CONSTRUCTION MANAGEMENT LABOR = $33.04 \%$, FDNH COHTRACT MANAGEMENT AHO ADMINISTRATION $=33.04 \%$

(3) FDH MPR/G\&A MATERIAL FACIOR: A COMPOSITE FACTOR OF $24.87 \%$ HÁS BEEN APPLIEO TO TOIAL FONH MATERIAL COST WHICH IHCLUDE A MPR OF $7 \%$ AND MATERIAL G\&A/FEE OF $16.7 \%$

\section{ESCALATIOI}

ESCALATIOH OERCENTAGES WERE CALCULATED FROM THE JANUARY 1997 UPOATE OF THE ECONOMIC ESCALATION PRICE CHANGE INDICES FOR DOE CONSTRUCTION PROJECTS AS PUBLISHEO BY THE "OFFICE OF INFRASTRUCTURE ACQUISITION" FH-5O.

5. CONTIHEENCY

A. DEFINITION OF CONTINGENCY AS PROVIDED BY DOE

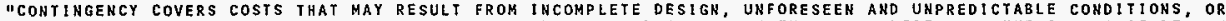
UNCERTALNTIES HITHIN THE DEFINED PROJECT SCOPE. THE AMOUHT OF CONTINGENCY WILL DEPEND OH THE STATUS OF DESIGH, PROCUREMENT, ANO CONSTRUCTION: AND THE COMPLEXITY ANO UNCERTAINTIES OF THE COMPONERT PARTS OF THE PROJECT. CONTINGENCY IS NOT TO BE USED TO AVOID MAKING AN ACCURATE ASSESSMENT OF EXPECTED COST" COFFICE OF NASTE MANAGEMENT (EN-3O) COST AND SCHEDULE GUIDE.

B. CONTIHGENGY ALLOHANCE GUIDELIHES

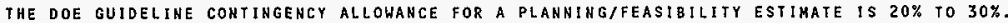

C. METHODOLOGY

CONTINGENCY IS EVALUATED AT THE LOWEST WORK BREAKOOWH STRUCTURE (UBS) LEVEL WITHIN THE COST ESTIHATE DETAILS. IT IS SUMMARIZED AT UPPER WBS LEVELS ANO REPORTED ON THE SUMMARY REPORTS.

D. ANALYSIS

AN ASSESSHENT OF DESIGN MATURITY, WORK COMPLEXITY AND PROJECT UNCERTAINIIES HAS BEEN PERFORHED. AN EXPLAINATION OF T.HIS ASSESSHEHT AND COHTIGENCY RATES WHICH HAVE BEEN ADDED TO THE COST OF WORK ARE AS FOLLOWS:

WBS 3 IXXXX CONSTRUCTION, ENGINEERING AND PROJECT MANAGEMENT - A 30\% CONTINGENCY HAS BEEN APPLIED TO COVER COSTS LIKELY TO SURFACE THAT HAVE NOT BEEN ANTICIPATED AT THIS EARLY STAGE OF PLAHNING. TECHNIQUES TO BE UTILIZED FOR DECONTAMINATION, THE WORX PROGRESS. 
FLUOR DANIEL NORTHWESI, INC. LOCXHEED MARIIN HANFORD CORP. JOB NO. E61945/F3RULS

FILE NO. 2475AADI

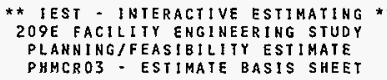

PAGE, 5 OF 9

DATE $06 / 16 / 97 \quad 14: 17: 07$

BY DKH/RWO

\section{REMARKS}

MAJOR ASSUMPTIONS HHICH HAVE BEEN hade IN THE PREPARATION OF thIS ESITHATE aRe as fOLlOHS:

A.) COSTS FOR CLOSURE CERTIFICATIONS, WIPE SAMPLES, SAMPLE ANALYTICAL COSTS, CORE SAMPLES, SOIL SAMPLES IECHNICAL PUBLICATIONS, VERIFICATION SAHPLING AND A DECOMMISIONING PLAN HAVE NOT BEEN ADDRESSED, AND II IHESE ARE REQUiRED, WILL MEED TO BE INCLUDED.

B.) LIHITED INFORMATION WAS AVAILABLE AND GROSS ASSUMPTIONS WERE MADE.

THE ESTIMATE ASSUMED THAT

Q.) ENGINEERING AND PROJECT MANAGEMENT COSTS HERE FIGURED AS A PERCENTAGE OF CONSTRUCTION, SEE ROB REPORT. 
FLUOR DANIEL NORTHHEST, INC.

LOCKHEED MARTIN HANFORD CORP.

JOB NO. E61945/F3RULS

FILE HO. Z475AAD

SORT

CODE/HBS

DESCRIPTION

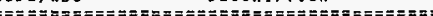

FDN

FLUOR DANIEL NORTHHEST

310300 ALT 3 - REMOVAL \& FIX IN PLACE TOTAL FDNH FLUOR DANIEL HORTHNEST

LMKC LOCKHEEO MARTIN HANFORD CORP.

310300 ALT. 3 - REMOVAL \& FIX IN PLACE TOTAL LMHC LOCKHEED MARTIN HANFORD COR
* IEST - IHIERACTIVE. ESTIMATING **

209E FACILITY ENGTHEERING STUDY
PLANAING/FEASIBILITY ESTI HATE ELTERHATIVE 3

PUMCRO 4 - COMPANYJWBS SUMMARY

ESTIMATE
SUBTOTAL

ESCALATION
$\%$ TOTAL

SUB

TOTAL

$\%$

ONT I NGENCY

$=x=\pi==$

$x=x== \pm===2$.

392520

392520

$30 \quad 117756$

117756

510276

PAGE

$\begin{array}{lll}\text { DATE } & 06 / 17 / 97 & 13: 39: 37\end{array}$

(1)

$264194 \quad 0.00$

$264194 \quad 0.00$

$==m==2==1=$

656,714

0.00

$==$

$==$

64194

$\begin{array}{ll}30 & 79258\end{array}$

$30 \quad 79258$

343452
343452

853,728

197,014

656,714 


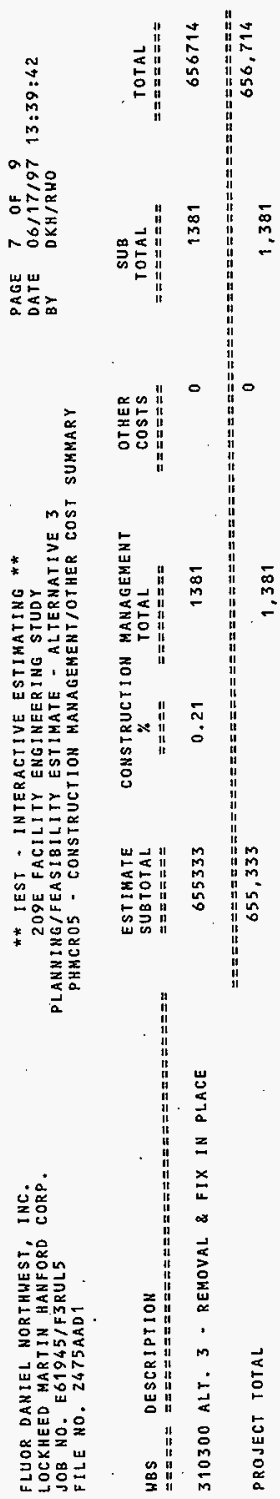




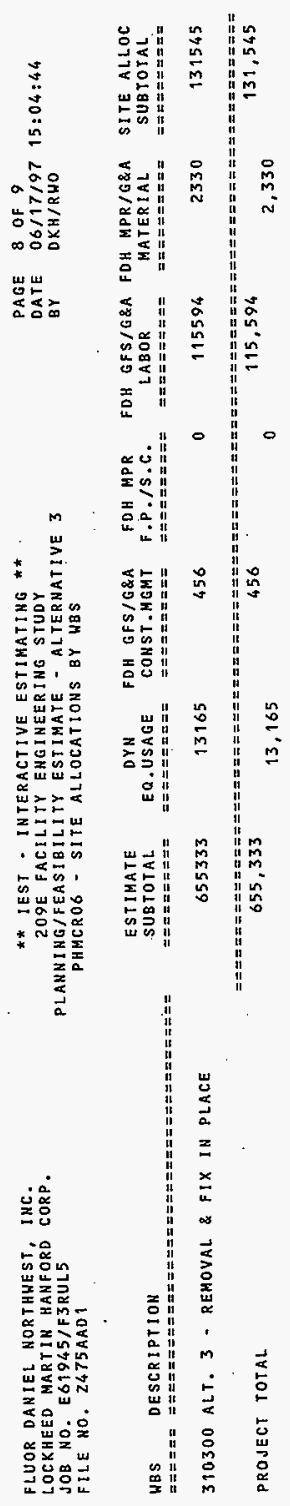


FLUOR DANIEL NORTHHEST, IMC.

LOCKHEED MART IN HANFORD CORP.

JOB HO. E61945/F3RUL 5

FILE NO. 2475AAD 1

WBS DESCRIPIION

WBS DERIPTION

310300 ALT. 3 - REMOVAL \& FIX IN PLACE

PROJECT TOTAL
* * IEST - INTERACTIVE ESTIMATING * *

209E FACILITY EHGINEERING STUDY

209E FACILITY EIIGINEERING STUDY PHACRO? - SITE ALLOCATION ESCALATION/CONTINGENCY REPORT

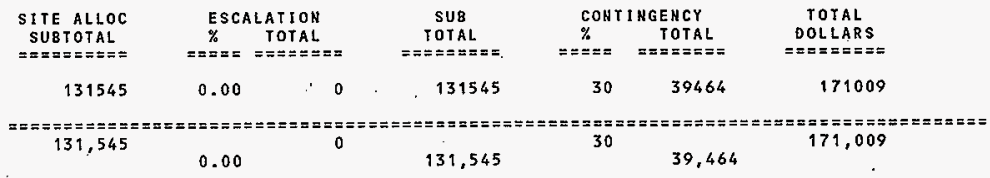


FLUOR DANIEL NORTHWEST, INC. LOCKHEED MARTIH HAHFORÓ CORP. JOB NO. E61945/F3RULS

FILE NO. Z475AAD 1

ACCOUNT
NUMBER DESCRIPTION

** IEST - INTERACTIVE ESTIMATING ** 209E FACILITY ENGINEERING STUOY
PLARNING/FEASIBILITY ESTIMATE . ALTERNATIVE PLANNINE/FEASIBILITY ESTIMATE - ALTERNATIVE $\begin{array}{lll}\text { PAGE } & 1 \\ \text { DATE } & 06 / 17 / 97 & 13: 39: 50\end{array}$

$\begin{array}{ll}\text { DATE } & 06 / 17 / 97 \\ \text { BY } & \text { DKH } / R W O\end{array}$ COST
CODE QUANTITY MANHOURS LABOR UQUIP
SUGE MATERIAL CONTRACT

310300 ALT. 3 - REMOVAL \& FIX IN PLACE

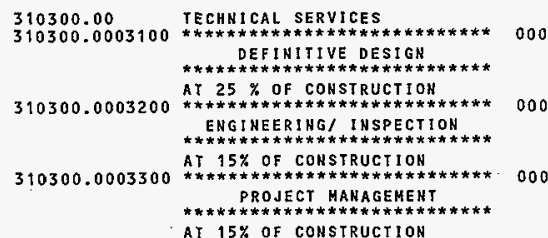

oo

$1 \mathrm{~L} / \mathrm{S} \quad 1667 \quad 110022$

0

0

0

0

0

110022

000

$1 \mathrm{~L} / \mathrm{S} \quad 1100 \cdot 66000$

o

o

0

0

o

66000

\section{0}

$1 \mathrm{~L} / \mathrm{S} \quad 904 \quad 65992$

o

0

o

0

65992

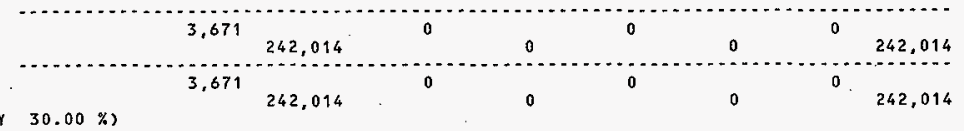

\subsection{1} GENERAL REQUIRMENTS

310300.0103226 BURIAL CHARGESFOR 3 BOXES 810 OF LLW $\$ 15.25 / C F$.

SUBTOTAL GENERAL REQUIRMENTS

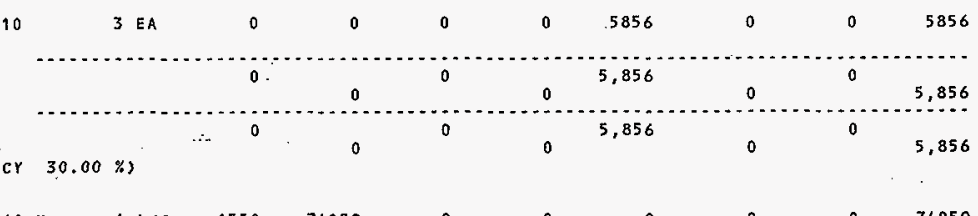

310300.01 GENERAL REQUIRMENTS

COST CODE 81001

HBS 310300

$0,00 \%$ - CONTINGENCY $30.00 \%$ GENERAL REQUIRMENTS
HPT TO MAN STEP OFF PAD

10300.0103000 HPT TO MAN STEP-OFF SUBTOTAL GEMERAL REQUIRMENTS SWP $100.00 \%$ IOTAL COST CODE 81001 HBS 310300

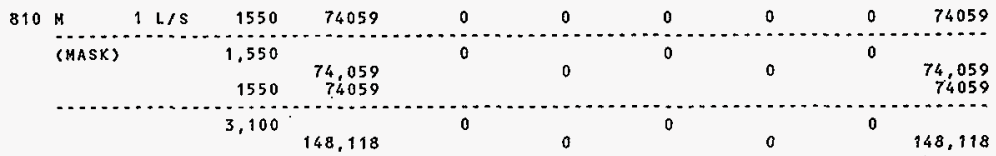


FLUOR DANIEL NORTHWEST, INC, LOCKHEED MARTIH HANFORD CORP.

ACCOUN

NUMBER

DESCRIPTION

ESCALATIOH

(ESCALATION

** IEST - INTERACTIVE ESTIMATING * 209E FACILITY ENGINEERING STUDY
PLANNIHG/FEASIBILIV PLANNIHG/FEASIBILITY ESTIMATE - ALTERNATIVE 3
PHMCRO8 - ESTIMATE DETAIL BY WBS / COST CODE

COST EOUIP

EQUIP SUB.

SU8 :

PAGE 2

DATE 06/17/97 13:39:50

DKH/RHO

$====$

CODE QUANTITY MANHOURS $L A B O R$

USAGE MATERIAL COHTRACT

EQUIP- OH\&P TOTAL

$========= \pm===0=0=0$

310300.02 SITEWORK

REMOVE EQUIPMENT

$810 \mathrm{M}$

310300.0203222 REMOVE ALL EQUIPUENT IN

GLOVE BOXES/HOODS, CUT UP

310300.0203224

GD PUT INTO BUR'IAL BOXES .

310300.0203224

DEC OB

$* * * * * * * * * * * * * * * * * * * * * * * * * * * * *)$

310300.0203241 HIPE DOWN GLOVE BOXES/HOODS AND FIX IN PLACE REMAINIMG CONTAMINATION.

310300.0203242 HIPE DOWH

310300.0203244

APPLY FIXATIVE, 50 HIL

EPOXY

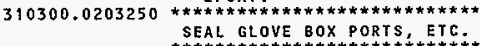
310300.0203252 SEAL GLOVE BOX PORTS,

310300.0203254 SEAL HOOD OPENINGS,

NSTALL CAPS AHD SEAL OFF.

310300.0203256 CUT AND CAP PIPES

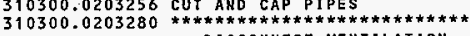
DISCONNECT VENTILATION

310300.0203282 DISCONNECT ACTIVE VENTIL. DISCONNECT ACTIVE VENTILATION TO

310300.0203290

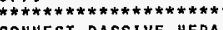

\section{CONNECT PASSIVE HEPA}

310300.0203292 CHECK PASSIVE HEPA FILTER

VENT ILATION SYSTEM TO GLOVE VENTILATION SYSTEM TO GLOVE BXXES/HTING.

310300.0203300

$\begin{array}{lllll}810 \mathrm{M} & 1 \mathrm{~L} / \mathrm{S} & 288 & 10944 & 0\end{array}$

$810 \mathrm{H}$

$810 \mathrm{H}$

810

$\begin{array}{rlr}810 & M & 1 \mathrm{~L} / \mathrm{S} \\ 810 & \mathrm{M} & 7000 \mathrm{SF}\end{array}$

$810 \mathrm{M}$

$810 \mathrm{M}$

$1 L / S$

$810 \mathrm{M}$

$810 \mathrm{M}$

$810 \mathrm{M}$

$810 \mathrm{M}$

$810 \mathrm{M}$

810

$1 \mathrm{~L} / \mathrm{s}$

$1 \mathrm{L/S}$

$1 \mathrm{~L} / \mathrm{s}$

0

$1 \mathrm{~L} / \mathrm{s}$

$810 \mathrm{M}$

\begin{tabular}{|c|c|c|c|c|c|c|c|}
\hline 0 & 0 & 0 & 0 & 0 & 0 & 0 & 0 \\
\hline 288 & 10944 & 0 & 0 & 0 & 0 & 0 & 10944 \\
\hline $\begin{array}{l}0 \\
0\end{array}$ & $\begin{array}{l}0 \\
0\end{array}$ & $\begin{array}{l}0 \\
0\end{array}$ & $\begin{array}{l}0 \\
0\end{array}$ & $\begin{array}{r}2000 \\
0\end{array}$ & $\begin{array}{l}0 \\
0\end{array}$ & $\begin{array}{l}0 \\
0\end{array}$ & $\begin{array}{r}2000 \\
0\end{array}$ \\
\hline 0 & 0 & 0 & 0 & 0 & 0 & 0 & 0 \\
\hline $\begin{array}{r}48 \\
588\end{array}$ & $\begin{array}{r}1311 \\
16458\end{array}$ & $\begin{array}{l}0 \\
0\end{array}$ & $\begin{array}{r}100 \\
4900\end{array}$ & $\begin{array}{l}0 \\
0\end{array}$ & $\begin{array}{l}0 \\
0\end{array}$ & $\begin{array}{l}0 \\
0\end{array}$ & $\begin{array}{r}1411 \\
21358\end{array}$ \\
\hline 0 & 0 & 0 & 0 & 0 & 0 & 0 & o \\
\hline 48 & 1737 & 0 & 250 & 0 & o & 0 & 1987 \\
\hline 50 & 1810 & 0 & 300 & 0 & 0 & 0 & 2110 \\
\hline $\begin{array}{r}80 \\
0\end{array}$ & $\begin{array}{r}3092 \\
0\end{array}$ & $\stackrel{0}{0}$ & $\begin{array}{r}250 \\
0\end{array}$ & $\begin{array}{l}0 \\
0\end{array}$ & $\begin{array}{l}0 \\
0\end{array}$ & $\begin{array}{l}0 \\
0\end{array}$ & $\begin{array}{r}3342 \\
0\end{array}$ \\
\hline 24 & 869 & 0 & 240 & 0 & 0 & 0 & 1109 \\
\hline 0 & 0 & 0 & 0 & 0 & 0 & 0 & 0 \\
\hline 16 & 579 & 0 & 0 & 0 & 0 & 0 & 579 \\
\hline
\end{tabular}

358

o

987

110

109

0

579 
FLUOR DANIEL NORTHUEST, IHC.

LOCKHEED MARTIN HANFORÓ CORP.

JOB NO. E61945/F3RUL5

FILE HO. Z475AAD I

ACCOUNT

NUMBER DESCRIPIION
* IEST - IMTERACTIVE ESTIHATING * * 209E FACILITY ENGIMEERIHG STUDY PLANNING/FEASIBTLITY ESTIMATE. ALTERNATIVE 3
PAGE $06 / 17 / 97 \quad 13: 39: 50$

DATE OK/TY/OT

\begin{tabular}{|c|c|}
\hline & 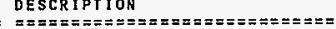 \\
\hline
\end{tabular}

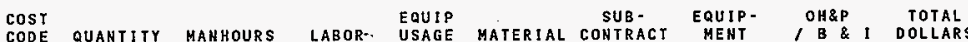

CODE QUANTITY MANHOURS LABORM USAGE MATERTAL CONTRACT MENT

AND 111 , FILL WITH GROUT.

$101,102,103,104,161$ AND 101.

310300.0203340 TANKS 105 AND 106 310300.0203344 CUT UP TAHKS 105\& 106 , PUTO BURIAL BOX

310300.0203346 BURIAL BOX

P

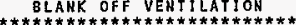

310300.0203362 BLANK FROM THE CAR AND HIX ROOM ON THE OUTSIDE OF BUILDING PAST

310300.0203364 SEAL UP INJECTIOH PORTS,
FERT HEPA FILTER. CALIBRATIOH PORTS, JOINIS AHD OTHER POSS 1 BLE EGRES POINTS IN DUCT WORK.

310300.0203380

S
SYSTEH SHUTDOHN $810 \mathrm{M} \quad 1 \mathrm{L/S} \quad 64 \quad 2304$

$810 \mathrm{M}$

$1, / s$

128

4608

100

$810 \mathrm{M}$

$810 \mathrm{M}$

1 EA

$810 \mathrm{M}$

$1 \mathrm{~L} / \mathrm{S}$

1 EA

810

$1 \mathrm{~L} / \mathrm{S}$

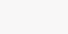

0

50

50
300

0

810

I

0
64
32

1920

5000

0
$500^{\circ}$
0

。

1000

0

1303

50

32

1158

100

$+2$

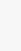

$810 \mathrm{M} \quad 0$

0
SHUT DOWN REMAINING SYSTEMS

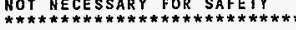

810 H 1 L/S 48

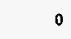

0

1728 CONTINUE CURRENT SURVEILLAHCE AND MAINTENANCE

$* * * * * * * * * * * * * * * * * * * * * * * * * * * *$ 310300.0203391 SURVEILLANCE AND MAINTENANCE
(209-E)

310300.0203392 SURVEILLANCE AND MAIHTENANCE

$810 \mathrm{H}$

0

$810 *$

$810 \mathrm{M}$

$1 \mathrm{~L} / \mathrm{S}$

45000

$1 \mathrm{~L} / \mathrm{S}$

10

500

9938 heALTh pHYSICS SURVEILLANCE

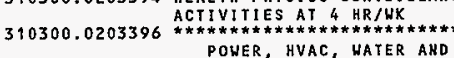
POWER, HVAC, WATER AND

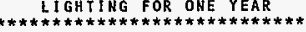

310300.0203398 ELECTRICAL UTILITY MAINTENAHCE

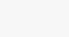


FLUOR OAHIEL NORTHWEST, IHC. LOCKHEED MARTIN HANFORD CORP. JOB NO. E61945/F3RULS

FILE NO. Z475AADI

ACCOUNT

NUNBER

DESCRIPYION

310300.0203399 BPA ELECTRICITY

SUBTOTAL SITEWORK

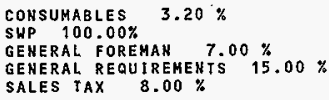

CONSUMABLES $3.20 \%$

SWP $100.00 \%$

GENERAL FOREMAN

$7.00 \%$

GENERAL REQUIREMEHTS $15.00 \%$

SALES TAX $8.00 \%$

TOTAL COST CODE 8100 ?

WBS 310300

SESCALATION

$0.00 \%$ - CONTINGENCY $30.00 \%$

* IEST - INTERACTIVE ESTIMATING *

209E FACILITY ENGIHEERING STUDY

PLANNING/FEASIBILTTY ESTIMATE. ALTERNATIVE 3

PHMCRO 8 - ESTIMATE DETAIL BY H8S / COST CODE

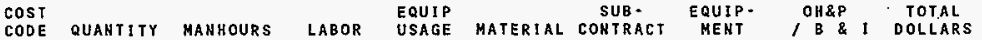

$810 \cdot \mathrm{H}$

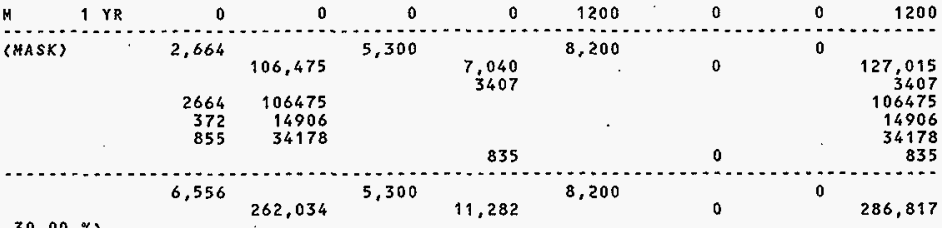

$\stackrel{D}{\omega}$

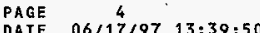

BY DKH $\angle$ RUO
TOTAL WES 310300 ALT. 3 - REMOVAL \& FIX IN PLACE
13,327

652,166
5,300

11,282

14,056

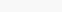

(1)

0

682,805 


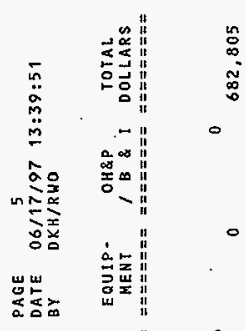

枈

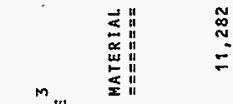

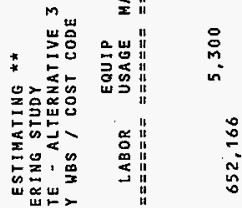

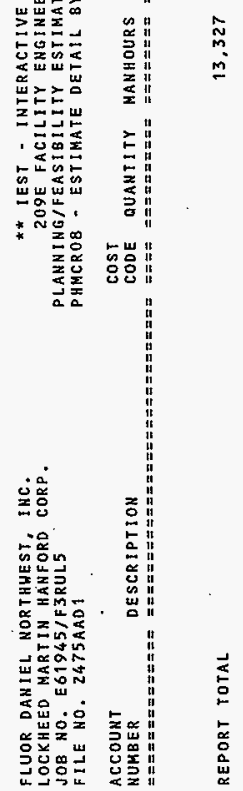


FLUOR DANIEL NORTHWEST, INC. LOCKHEED MARTIN HANFORD CORP. JOB NO. E61945/F3RULS FILE NO. 2475 AAEI

\section{** IEst - interacilive estIMating * * \\ 209E FACILITYY ENGINEERIHG STUDY \\ PLANNIHG/FEASIBILITY ESTIMATE. ALT \#4, SIAGE \\ PHMCRO1. PROJECT COSI SUMMARY}

PAGE 1 OF 9

$13: 49: 35$

\begin{tabular}{|c|c|}
\hline $\begin{array}{l}\text { SORT } \\
===\approx==E=\end{array}$ & $\begin{array}{l}\text { OE SCR IPT ION } \\
======z===== \pm= \pm\end{array}$ \\
\hline FDNH & FLUOR DANIEL NORT \\
\hline LMHC & LOCKHEED MARTIN \\
\hline & OTAL \\
\hline SIIE & SITE ALLOCATIONS \\
\hline
\end{tabular}

\begin{tabular}{|c|c|c|c|}
\hline $\begin{array}{l}\text { ESCALATED } \\
\text { TOTAL COST } \\
==========\end{array}$ & $==\%^{\operatorname{con}}$ & $\begin{array}{l}\text { NGENCY } \\
\qquad \begin{array}{l}\text { TOTAL } \\
==\approx=\approx=====\end{array}\end{array}$ & $\begin{array}{c}\text { TOIAL } \\
\text { DOLLARS } \\
====\equiv=====\end{array}$ \\
\hline 597.979 & 30 & 179,394 & 777,373 \\
\hline 180,418 & 30 & 54,125 & 234,543 \\
\hline
\end{tabular}

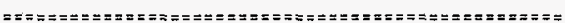

\begin{tabular}{|c|c|c|c|}
\hline 778,397 & 30 & 233,519 & $1,011,916$ \\
\hline 196.135 & 30 & 58,840 & 254,975 \\
\hline
\end{tabular}

$\prod_{0}^{\infty}$

PROJECT TOTAL

974,532

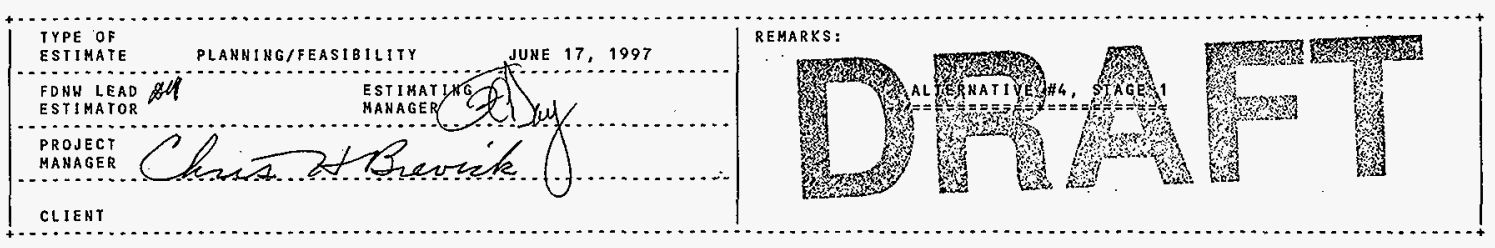


FLUOR DANIEL NORTHHEST, INC.

LOCXHEED MARYIN HANFORD CORP.

FLE NO. Z475AAE1

HBS DESCRIPTION

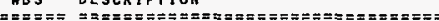

310410 ALTERNATIVE 4 - STAGE

PROJECT TOTAL
* IEST - INTERACTIVE ESTIMATING **

209E FACILITY ENGINEERING STUDY

PLANNING/FEAS IBILITY. ESTIMATE - ALT \#4, STAGE
PHMCROZ - WORK BREAKDONH STRUCTURE (HBS) SUMMARY

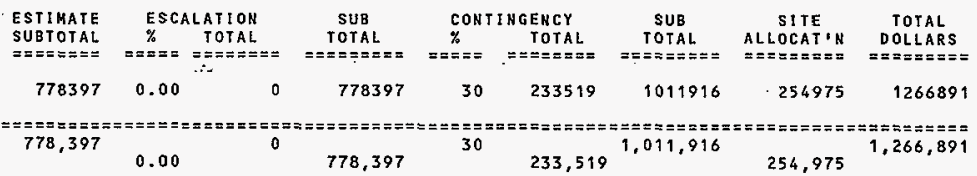


FLUOR DANIEL NORTHHEST, INC.

LOCKHEED MARTIN HANFORD CORP.

JOB NO. E61945/F3RULS

FILE NO. Z475AAE 1

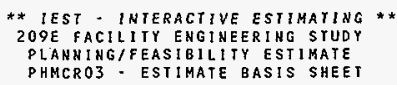

209E FACILITY ENGINEERING STUDY

PHMCRO3 . ESTIMATE BASIS SHEET
PAGE 3 OF 9

$14: 17: 07$ DKH/RWO

1. ESTIMATE PURPOSE

THIS ESTIKATE WILL BE USED FOR A SCOPING STUDY.

2. ESTIMATE TECHNICAL BASIS

A. THIS ESTIMATE HAS BEEN PREPARED FOR LOCKHEED MARIIH YANEORD CORP AS REQUESTED BY FDNH PROJECT MANAGER

B. A ROUGH DESCRIPTION OF THE SCOPE OF HORK HAY BE FOUND IN THE OUTLINE OF ALTERHATIVES. AM UNDATED AHD

UNNUMBERED DOCUMENT.

THE FOLLOWING CONSTRAINTS AND/OR SPECIAL CONDITIONS EXIST: ALL HORX DONE. IN THE CRITICAL ASSEMBLY ROOM AND IN THE HIXING ROOM HILL REQUIRE GOLLOWING THE APPROPRIAIE RADIATION WORK PROCEDURE.

3. ESTIMATE METHODOLOGY

A. DIRECT COSTS

A MANLOADIHG TECHATQUE HAS USED TO COME UP WITH THE LABOR HOURS WHEN NECESSARY DUE TO THE UHDEFINED SCOPE.

(1) CONSTRUCTION LABOR. MATERIAL AND EQUIPMENT UNITS HAVE BEEN ESTIMATED BASED UPOH OHE OR HORE OF THE FOLLOUING STANDARO COMMERCIAL ESTIMATING RESOURCES, PUBLISHED ESTIMATING MANUALS AND R.S. MEANS

B. DIRECT COST FACTORS

(1) SALES TAX HAS BEEH APPLIED TO ALL MATERIALS AND EQUIPHENT PURCHASES AT 8\%.

(2) NO HAREHOUSING COSTS ARE SHONN SINCE THEY ARE CONSIDERED TO BE INCLUDED IN THE MATERIAL PROCUREMENT RATE (MPR).

(3) AN ESTIMATING FACTOR OF 15\% HAS 8EEN APPLIEO TO DIRECT CRAFT LABOR FOR GEHERAL REQUIREMENTS AND $23.58 \%$ FOR TECHNICAT SERVICES.

(5) SPECIAL NORK PROCEDURE (SUP) FACTORS ARE APPLIED AGAINST DIRECT LABOR FOR ACTUAL TIME LOST DUE TO THE PERSONREL PROTECTIVE EQUTPMENT AND PROCEDURES. THE RATES HHICH HAVE BEEN APPLIED ARE AS FOLLOHS:

PROTECTJVE CLOTHING FACTOR HAS BEEN APPLIED AT $15 \%$

CONTAMINATION RESTRICTIONS ASSOCIATED WITH RADIATION DOSE LIMITS (BURNOUT) HAVE NOT BEEN CALCULATEO AS IT IS NOT BELIEVE TO BE A CONCERN FOR THESE ACTIVITIES.

(6) PREMIUM PAY

OVERTIME REQUIREMENTS AND SHIFI DIFFERENTIAL PAY FOR CRAFT LABOR WILL NOT BE REQUIRED FOR THIS WORK, AS STRAIGHI TIME, 40 HOUR WEEKS ARE DEEMED ADEQUATE TO ACCOMPLISH THIS HORK.

(7) GENERAL FOREMAN FACTOR OF TU HAS BEEU APPLIED TO DIRECT

(8) A FACIOR OF $10 \%$ HAS BEEN APPLIED TO DIRECT CRAFT LABOR TO ALLOH FOR USAGE OF GOVERNHENT OHNED EQUIPHENT CONTROLLEO BY OYNCORP.

c. RATES

(1) FLUOR DANIEL NORTHWEST LABOR RATES ARE BASED UPOY THE FLUOR DALNEL FEDERAL OPERATIONS (FEDFO) DISCIOSURE STATEMENT AND APPROVED PROVISIONAL BILLING RATES. FOR ESTIMATING PURPOSES, AVERAGE RATES BY OPERATIONS CODE HAVE BEEN

(2) FLUOR DANIEL NORTHHEST SERVICES (CONSTRUCTIOH CRAFI LABOR) RATES ARE THOSE LISTED IN APPENDIX A TO THE HANFORD SITE

STABILLIZATION AGREEMEHT

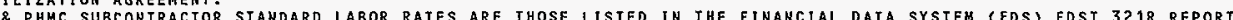
ORGAMIZATION RATES PLUS ADDERS. 
FLUOR DANIEL NORTKHEST, INC. LOCKHEED MARTIN HANFORD CORP. JOB NO. E61945/F3RULS

FILE NO. Z475AAEI

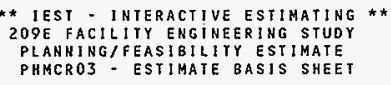

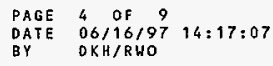

D. SITE ALLOCATIONS FACTORS

SITE RLLOCATION FACIORS ARE DEVELOPED AND PROVIDED BY FLUOR DARIEL HANFORD (FDH) FOR ESTIMATING USE.

(1) GOVERHMENT FURNISHED SERVICES RATE IS APPLIED TO ALL COSTS TO LIQUIDATE GOVERNAENT FURMISHED SERVICES PROVIDED TO THE ENTERPRISE COMPANIES: $14 \%$ FOR FDNW, $10 \%$ FOR FDNUS (CONSTRUCTIOH)

(2) HALFORD SIIE G\&A RAIE OF $16.7 \%$ IS APPLIED TO ALL COSTS TO LIQUIDATE THE HANFORD GEMERAL \& ADMINISTRAIIVE COSTS .

(3) HANFORD SITE MPR RATE OF $7.0 \%$ IS APPLIED TO ALL PURCHASED HATERIAL AND $7.7 \%$ TO ALL PURCHASED SERICES TO LIQUIDAIE THE COST OF PROCUREMENT (INCLUDING RECIEVING).

FONW APPLIES THE ABOVE FACTORS TO ESIIHATED COSTS AS FOLLOWS:

(1) FDH GFSIG\&A CM FACTOR: A COMPOSITE FACTOR OF 33.04\% HAS BEEN APPLIED TO TOTAL FDNW FIXEO PRICE CONSTRUCTION

MANAGEMENT WHICH INCLUDES GOVERMMENT FURNISHED SERVICES (GFS) AND SITE G\&A/FEE.

(2) FOH GFS/G\&A LABOR FACTOR: A COMPOSITE FACTOR HAS BEEN APPLIEO TO TOTAL FDHW LABOR COSTS AS FOLLOWS:

AE $/ C M$ COSTS $=33.04 \%$, FDNHS CONSTRUCTION LABOR = $28.37 \%$, FDNWS COUSTRUCIION MANAGEMENT LABOR = $33.04 \%$, FDHH CONTRACI

(3) FDH MPR/G\&A MATERIAL FACTOR: A COMPOSITE

4. ESCALATION

ESCALATION PERCENTAGES HERE CALCULATED FROM THE JARUARY 1997 UPDATE OF THE ECONOMIC ESCALATION PRICE CHANGE INDICES FOR DOE ESCALTRUTI ON PROJECTS AS PUBLISHED BY THE "OFFICE OF INFRASTRUCTURE ACQUISITION" FM-5O.

5. CONTINGENCY

A. DEFINITION OF CONTINGENCY AS PROVIDED BY DOE

"CONTINGENCY COVERS COSTS THAT MAY RESULT FROM INCOMPLETE DESIGN, UNFORESEEN AND UNPREDICTABLE CONDITIONS, OR UNCERTAINTIES WITHIN THE DEFINED PROJECT SCOPE. THE AMOUHT OF CONTINGENCY HILL DEPEND ON THE STATUS OF DESIGN, PROCUREMEHT. AND CONSTRUCTION; AND THE COMPLEXITY AND UNCERTAINTIES OF THE COMPONENT PARTS OF THE PROJECT. CONTIHGENCY IS NOT TO BE USED TO AVOID MAKING AN ACCURATE ASSESSMENT OF EXPECTED COST" (OFFICE OF WASTE MANAGEMENT (EM-3O) COST AND SCHEDULE GUIDE.

B. Contingency allohance guidelines

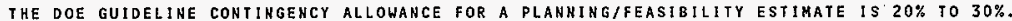

c. HETHODOLOGY

CONTINGENCY IS EVALUATED AT THE LOWEST HORK BREAKDOWN STRUCTURE (WBS) LEVEL WITHIN THE COST ESTIMATE DETAILS. IT IS SUMMARIZED AT UPPER WBS LEVELS AHD REPORYED ON THE SUMMARY REPORTS.

D. ANALYSIS

AN ASSESSHENT OF DESIGN MATURIIY, HORX COMPLEXITY AND PROJECT UNCERTAINTIES HAS BEEN PERFORMEO. AN EXPLAINATION OF THIS ASSESSMENT AND CONTIGENCY RATES WHICH HAVE BEEN ADDED TO THE COST OF WORK ARE AS FOLLOHS:

HBS 31 XXXX CONSTRUCTION, ENGTHEERING AND PROJECT MANAGEMENT - A $30 \%$ CONTINGENCY HAS BEEN APPLIED TO COVER COSTS LIKELY TO SURFACE THAT HAVE NOT BEEN ANTICIPATED AT THIS EARLY STAGE OF PLANHING. TECHNIQUES TO BE UTILIZEO FOR DECONTAMINATION, SIABILIZATIOH AND REMOVAL HILL HEED FURTHER STUDY AND MANY OF THE ACTIVITIES WILL BE SUBJECT TO CHANGE AT ANY TIME DURING
THE WORX PROGRES. 
FLUOR DANIEL NORTHUEST, INC. LOCKHEEO MARTIN HAHFORD CORP.

JOB NO. E61945/F3RULS

FILE HO. 2475AAE1
* IEST - INTERACTIVE ESTIMATINg * *
$209 E$ FACILITY ENGINEERING STUOY
PLANAING/FEASIBILIITY ESTIMATE
PHMCRO3 - ESTIMATE BASIS SHEET

$\begin{array}{lll}\text { PAGE } & 5 \text { OF } 9 & \\ \text { DATE } & 06 / 16 / 97 & 14: 17: 07\end{array}$

6. REMARKS

MAJOR ASSUMPTIONS WHICH HAVE BEEN HADE IN THE PREPARATION OF THIS ESITMATE ARE AS FOLLOHS:

A.) COSTS FOR ClOSURE CERTIFICATIONS, WIPE SAMPLES, SAMPLE ANALYIICAL COSIS, CORE SAMPLES, SOIL SAMPLES,

IECHNICAL PUBLICATIONS, VERIfICAJION SAMPLING ANO A DECOMMISIONING PLAN HAVE NOT BEEN ADDRESSED, AND IF IHESE ARE REQUIRED. WILL NEEO TO BE INCLUDED.

B.) LIMITED INFORMATLON WAS AVATLABLE ANO GROSS ASSUMPTIONS HERE MADE.

C.) THE ESTIMATE ASSUMED THAT BURN - OUT WOULD NOT BE ENCOUNTERED 
FLUOR DANIEL NORTHHEST, 1 NC.

LOCKHEED MARTIN HANFORD CORP.

JOB NO. E61945/F3RUL5

FILE NO. Z Z75AAE

SORT

CODE/WBS

DESCRIPTION

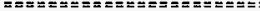

FONW FLUOR DANIEL NORTHUEST

310410 ALTERNATIVE 4 - STAGE

TOTAL FDNW FLUOR DANIEL NORTHWEST

LMHC LOCKHEED MARTIN HANFORD CORP.

310410 ALTERHATIVE 4 . STAGE 1

TOTAL LMHC LOCKHEED MARTIH HANFORD COR

PROJECT TOTAL
* IEST - INTERACt IVE ESTIMATINg **

209E FACILITY ENGINEERING STUDY PLAHAING/FEASIBILITY ESTIMATE - ALI \#4, STAGE

PHMCRO4: COMPANYIWBS SUMMARY

$\begin{array}{llll}\text { PAGE } & 6 & \text { OF } & 9 \\ \text { DATE } & 06 / 17 / 97 & 13: 49: 41\end{array}$

BY DXH/RHO

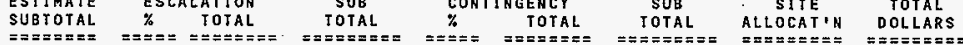

$597979 \quad 0.09$

$0.00 \quad \cdot 0$

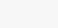

(30

30

179394

179394
179394

777373
777373

254975

254975
254975

1032348

1032348

$\begin{array}{lllllllll}180418 & 0.00 & 0 & 180418 & 30 & 54125 & 234543 & 0 & 234543 \\ 180418 & 0.00 & 0 & 180418 & 30 & 54125 & 234543 & 0 & 234543\end{array}$

$==$

778,397

30

233,519

254,975 


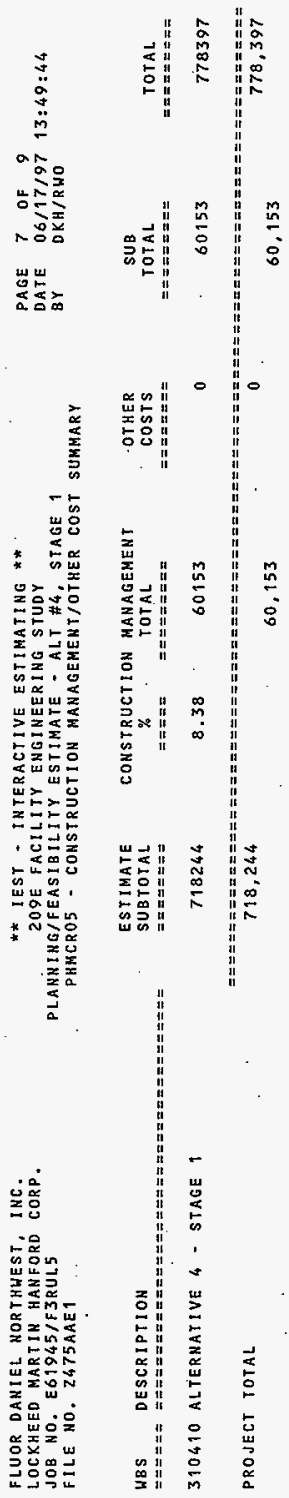




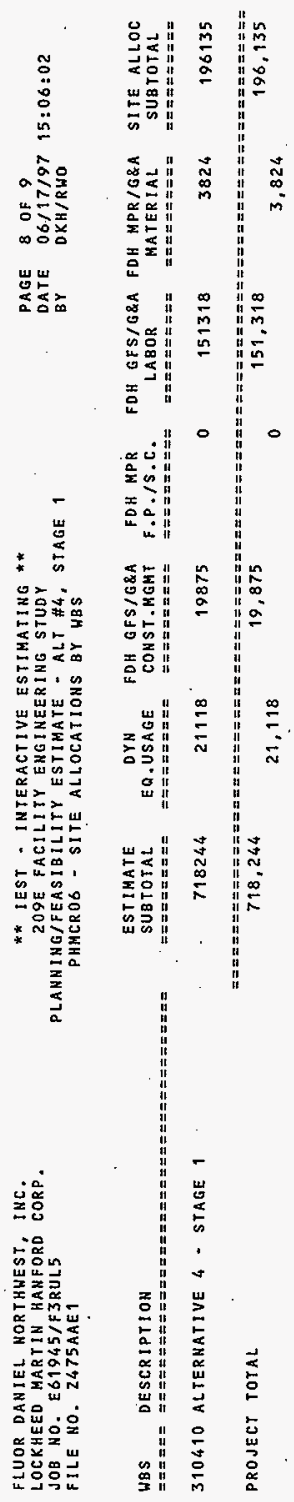


FLUOR DANIEL, NORTHHEST, INC.

LOCKHEED MARTIN HANFORD CORP.

JOB HO. E61945/E3RUL5

FILE NO. 2475 AAE 1

WBS DESCRIPTION

Ription

310410 ALTERHATIVE 4 - STAGE

PROJECT TOTAL
* IEST - INTERACTIVE ESTIMATIHG *

209E FACILITY ENGTMEERING STUDY

PLANNING/FEASIBILITY ESTIMATE - ALT \#4, STAGE PHMCROT - SITE ALLOCATION ESCALATION/CONTINGENCY REPORT
PAGE 9 OF 9

$06 / 47 / 97 \quad 13: 49: 47$

BY DKH/RWO

\section{SITE ALLOC} SUBTOTAL

196135 ESCALATION
$\%$ TOTAL

0.00

$==\pi===\pi==$
0.00

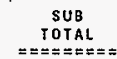

196135

\begin{tabular}{|c|c|c|}
\hline \multicolumn{2}{|c|}{ CONTINGENCY } & TOTAL \\
\hline * & TOTAL & DOLLARS \\
\hline & 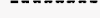 & $==-=$ \\
\hline 30 & 58840 & 254975 \\
\hline
\end{tabular}

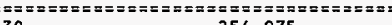

$===2==$
254,975 
FLUOR DANIEL NORTHWEST, INC.

OCKHEED MARTIN HANFORD CORP.

EILE NO, 2475AAE 1

\section{AT $15 \%$ OF CONSTRUC
SUBTOTAL TECHAICAL SERVICES \\ TORAL COST CODE 00000}

WES 3104100000 310410.01 GENERAL REQUIRMENTS 310410.0104066 BURIAL CHARGES FOR
OF LLH O $\$ 15.25 / \mathrm{CF}$

\section{Subtotal geNeral requirments}

TOTAL COST CODE 81001

WBS 310410

(ESCALATION 0.00\% - CONTINGEMCY $30.00 \%$

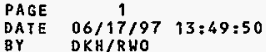

209E FACILITY ENGINEERING STUDY

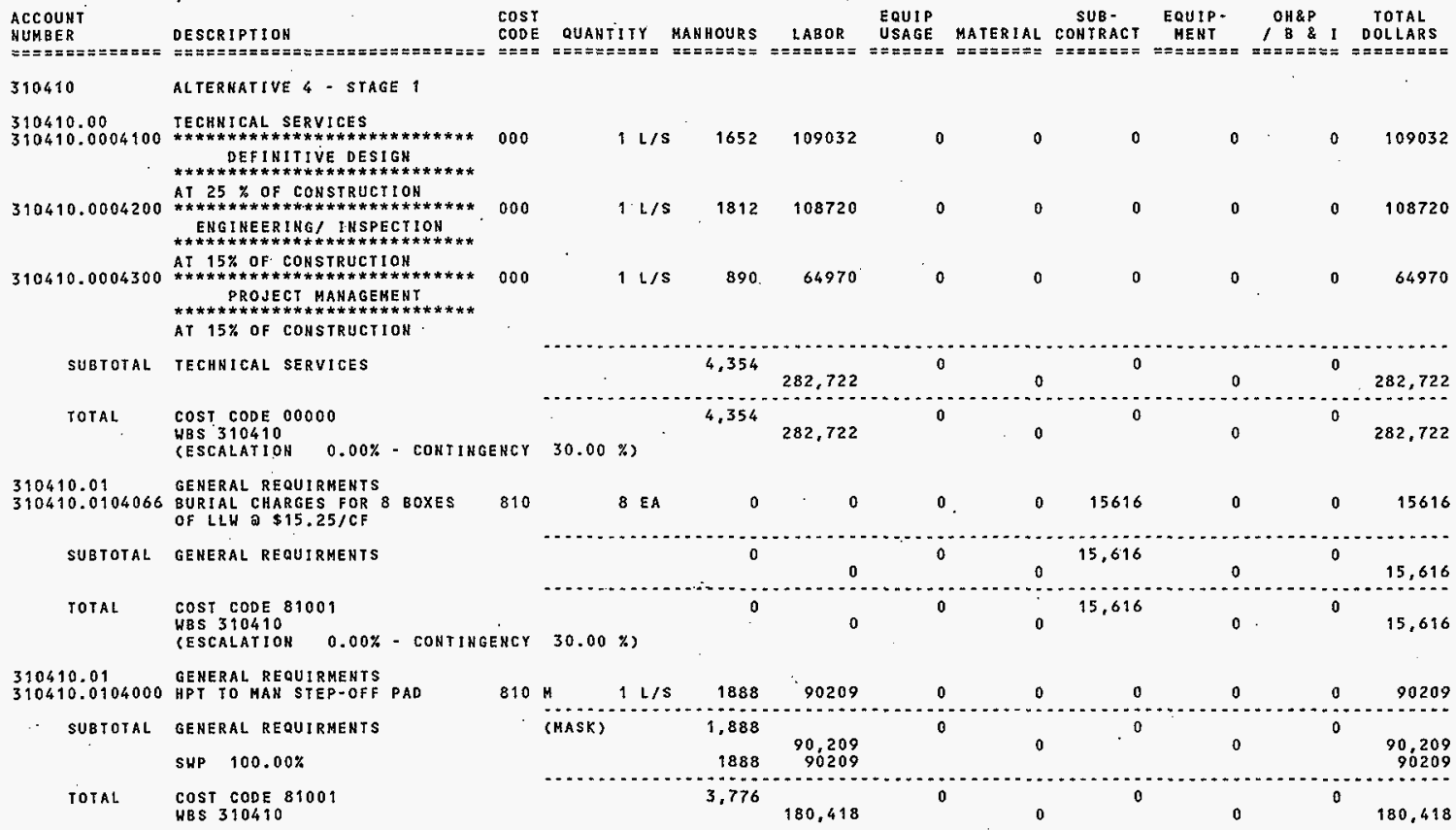




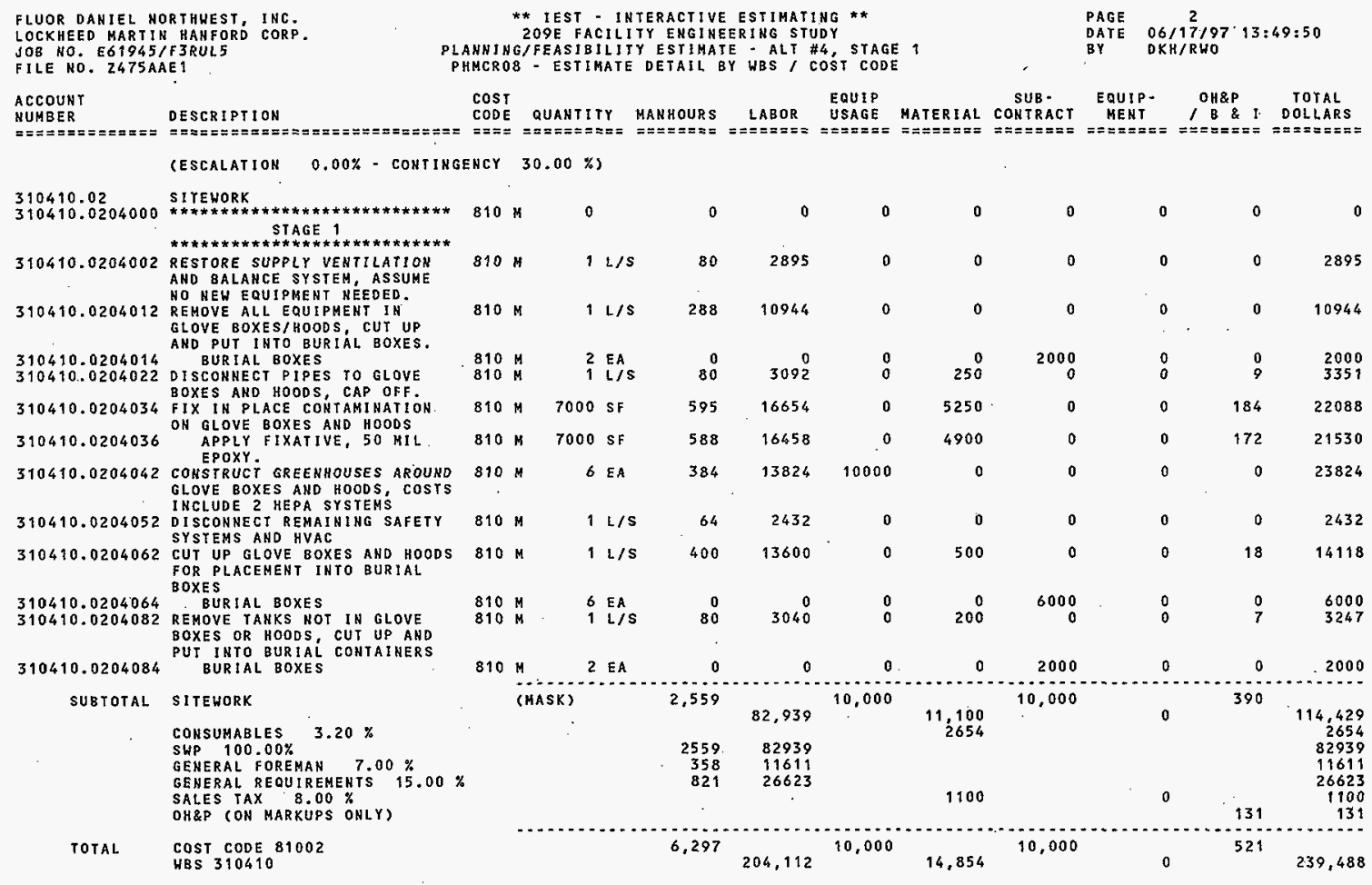


FLUOR DANIEL NORTHWEST, INC.

LOCKHEED MARTIN HANFORD CORP.

JOB HO. E61945/F3RUL5

FILE NO. 2475AAE

ACCOUNT

NUMBER

DESCRIPTIOH

(ESCALATION

* IEST - INTERACTIVE ESTIMATING *

209E FACILITY ENGINEERING STUDY PLANNING/FEASIBILITY ESTIMATE - ALT \#4, STAGE PHMCROB - ESTIMATE OETAIL BY WBS/ COST CODE

\section{PA}

DATE $06 / 17 / 97 \quad 13: 49: 51$ $\cos$

QUANTITY MANHOURS LABOR UQUIP USAE MATERIAL CONTRAC

MATERIAL CONTRCT EQUIP. OH\&P TOTAL USAGE MATERIAL CONIRACT MENT I 8 \& I DOLLARS

$30.00 \%$

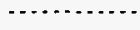

14,427
$667,252 \quad 10,000$
14,854
25,616
521

718,244 
FLUOR DAMIEL HORTHHEST, IHC.

LOCKHEED MART IN HANFORD CORP.

JOB NO. E61845/F3RUL5

* IEST - INTERACTIVE ESTIMATIHG **

EILE NO. 2475AAEI

ACCOUNT

NUMBER

DESCRIPTIOH

REPORT TOTAL

667,252

14,854

$\begin{array}{lll}\text { PAGE } & 4 \\ \text { DATE } & 06 / 17 / 97 & 13: 49: 51\end{array}$ PLANNING/FEASIBILITY ESTIMATE - ALT \#4 STAGE PHMCROS - ESTIMATE DEJALL BY HBS I COST CODE

USAGE

MATERIAL CONTRACT

BY OKH/RHO

EQUIP- OH\&P TOTAL MENT $I B$ \& I DOLLARS

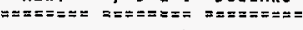


FLUOR DANIEL HORTHHEST, INC. LOCKREED MARTIN HANFORO CORP. JOB NO. E6IS45/F3RULS FILE WO. Z475AAF 1

209E EACILITY ENGINEERING STUDY

PLANNINGJFEASIBILIIY ESTIMATE - ALT \#4, SIAGE PHMCRO1 - PROJECT COST SUMMARY $\begin{array}{ll}\text { PAGE } & 1 \text { OF } 9 \\ \text { DATE } & 06 / 17 / 97 \\ \text { BY } & \text { DXH/RHO }\end{array}$

OTAL

DOLLARS

\begin{tabular}{|c|c|c|c|}
\hline \multirow{3}{*}{$\begin{array}{l}\text { ESCALATED } \\
\text { IOTAL COST } \\
=\approx=======\end{array}$} & \multicolumn{2}{|c|}{ COHTINGENCY } & \multirow{3}{*}{$\begin{array}{c}\text { IOTAL } \\
\text { DOL LARS } \\
==========\end{array}$} \\
\hline & $\%$ & TOTAL & \\
\hline & $=\pi=\pi=$ & 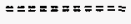 & \\
\hline 52,302 & 30 & 15,690 & 67.992 \\
\hline 7,644 & 30 & 2,293 & 9,937 \\
\hline
\end{tabular}

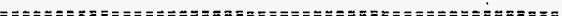

$\begin{array}{llll}59,946 & 30 & 17,983 & 77,929\end{array}$

$\begin{array}{llll}16,335 & 30 & 4,901 & 21,236\end{array}$

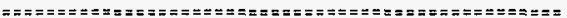

76,281

22,884

99,165

PROJECT TOTAL

(2) 
FLUOR DANIEL NORTHHEST, INC,

LOCKHEED MARTIN HANFORD CORP.

SOB NO. E61945/F3RULS

FILE NO. 2475AAF 1

WBS DESCRIPIION

WB $=x=$

310420 ALTERHATIVE 4 - STAGE 2

PROJECT TOTAL
* \# IEST - INTERACTIVE ESTIMATING **

209E EACILITY ENGIHEERING STUDY

PLANNING/FEASIBILITY ESTIMATE. ALT \#4, STAGE PHMCRO2 - WORK BREAKDOWN STRUCTURE (WBS) SUMMARY
PAGE 2 OF 9

$714: 05: 54$

BY DKH/RHO

\begin{tabular}{|c|c|c|c|c|c|c|c|c|}
\hline $\begin{array}{l}\text { ESTIMATE } \\
\text { SUB TOTAL } \\
=======\end{array}$ & $\begin{aligned} & \text { ESCA } \\
& \% \\
& \%===\end{aligned}$ & $\begin{array}{l}\text { LATION } \\
\text { TOTAL } \\
\Rightarrow= \pm===\approx\end{array}$ & $\begin{array}{c}\text { SUB } \\
\text { I0TAL } \\
===\approx=\approx===\end{array}$ & $\begin{array}{l}\operatorname{CONT} \\
\% \\
=\pi==\end{array}$ & $\begin{array}{l}\text { INGENCY } \\
\text { TOIAL } \\
\Rightarrow==\pi==\approx=\end{array}$ & $\begin{array}{c}\text { SUB } \\
\text { rOTA } L \\
\Rightarrow==x====\end{array}$ & $\begin{array}{c}\text { SITE } \\
\text { ALLOCAT'N } \\
===\approx=====\end{array}$ & $\begin{array}{c}\text { TOTAL } \\
\text { DOLLARS. } \\
=\approx======\equiv\end{array}$ \\
\hline 59946 & 0.00 & 0 & 59946 & 30 & 17983 & 77929 & 21236 & 99165 \\
\hline \multicolumn{9}{|c|}{ 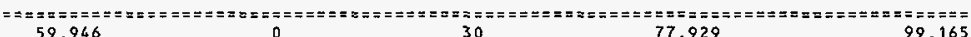 } \\
\hline 59,946 & 0.00 & 0 & 59,946 & 30 & 17,983 & 77,929 & 21,236 & 99,165 \\
\hline
\end{tabular}

品 
FLUOR DANIEL NORTHLEST, INC. LOCKHEED MARTIN HAHFORD CORP. JO8 NO. E61945/F3RUL5

FILE HO. Z475AAF

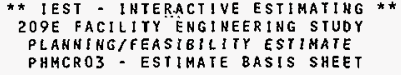

PAGE 3 OF 9

DATE $06 / 16 / 97 \quad 14: 17: 07$

BY DKH/RHO

1. ESTIMATE PURPOSE

THIS ESTIMATE HILL BE USED FOR A SCOPING STUDY.

2. ESTIMATE TECHNICAL BASIS

A. THIS ESTIMATE HAS BEER PREPARED FOR LOCKHEED MARTIN HANFORD CORP. AS REQUESTED BY FDNH PROJECT MANAGER.

B. A ROUGH DESCRIPTION OF THE SCOPE OF HORK MAY BE FOUND IH THE OUILIHE OF ALIERHATIVES, AN UNDATED AND

C. UNHUMBERED DOCUMENT.

C. THE FOLLOWING CONSTRAINTS AND/OR SPECIAL CONDITIONS EXIST: ALL HORK DONE IN THE CRITICAL ASSEMBLY ROOM AND IN THE MIXING ROOM WILL REQUIRE FOLLOHIWG THE APPROPRIATE RADIAIION HORK PROCEDURE.

3. ESTIMATE METHODOLOGY

A. OIRECT COSTS:

A MANLOADING TECHNIQUE HAS USED TO COME UP UITH THE LABOR HOURS UHEN HECESSARY DUE YO THE UNDEFINED SCOPE.

(1) COHSTRUCION LABOR MATERIAL ANO EQUIPMENT UNITS HAVE BEEN ESTIMATED BASED UPON ONE OR MORE OF THE FOLLOWING STANDARD CONSTRUCTION LABOR, HATERIAL ANO EQUIPHENT UNITS HAVE BEEN ESTIMATED BASED UPO
COMMERCIAL ESTIMATIMG RESOURCES. PUBLISHED ESTIMATING MANUALS ANO R.S. MEANS

B. DIRECT COST FACTORS

(1) SALES TAX HAS BEEN APPLIED TO ALL MATERIALS AND EQUIPMENT PURCHASES AT $8 \%$

(2) NO WAREHOUSING COSTS ARE SHOWN SINCE THEY ARE CONSIDERED TO BE INCLUDED IN THE MATERIAL PROCUREMENT RATE (MPR).

(3) AN ESTIMATING FACTOR OF 15\% HAS BEEN APPLIED TO DIRECT CRAFT LABOR FOR GENERAL REQUIREMENTS AND

23.58\% FOR TECHNICAL SERVICES

(4) CONSUMABLES ARE ESTIMATED AT $3.2 \%$ OF OIRECT CRAFT LABOR COSTS

(5) SPECIAL HORK PROCEDURE (SHP) FACTORS ARE APPLIED AGAINST DIRECT LABOR FOR ACTUAL IIME LOST DUE TO THE PERSOHNEL PROTECTIVE EQUIPHENT AND PROCEDURES. THE RAIES HHICH HAVE BEEN APPLIED ARE AS FOLLOWS: PROTECTIVE CLOTHING FACTOR HAS BEEN APPLIED AT $15 \%$ MASK HORK AT $85 \%$ PLUS $15 \%$ FOR PROTECIIVE CLOTHING. CONTAMINATION RESTRICTIONS ASSOCIATED WITH RADIATION DOSE LIMITS (BURNOUT) HAVE HOT BEEN CALCULATED AS IT IS NOT BELIEVE TO BE A CONCERN FOR THESE ACTIVITIES.

(6) PREMIUM PAY

OVERTIME REQUIREMENTS AND SHIFI DIFFERENTIAL PAY FOR CRAFT LABOR HILL HOT BE REQUIRED FOR THIS. WORK, AS SIRAIGHT TIME, 40 HOUR WEEKS ARE DEEMED ADEQUATE TO ACCOMPLISH THIS WORK.

(8) A FACTOR OF $10 \%$ HAS BEEM APPLIED TO DIRECT CRAFT LABOR IO ALLOH FOR USAGE OF GOVERNMENT OHAED EQUIPMENT CONTROLLED BY DYHCORP.

C. RATES

(1) FLUOR OAHTEL NORTHUEST LABOR RATES ARE BASED UPON THE FLUOR DAIMEL FEDERAL OPERATIONS (FEDFO) DISCLOSURE STATEMENT AND APPROVED PROVISIONAL BILLING RATES. FOR ESTIMATING PURPOSES, AVERAGE RATES BY OPERATIOHS CODE HAVE BEEN DEVELOPED 8 ASED UPON RECENT COST HISTORY

(2) FLUOR DANIEL NORTHUEST SERVICES (CONSTRUCTION CRAFT LABOR) RATES ARE THOSE LISTED IN APPENDIX A TO THE HANFORD SITE STABILIZATION AGREEMENT.

(3) FDH \& PHMC SUBCONTRACTOR STANDARD LABOR RATES ARE THOSE LISTED IN THE FINANCIAL DATA SYSTEM (FDS) FDST $321 R$ REPORT ORGAHIZATION RATES PLUS ADDERS. 
FLUOR DANIEL NORTHWEST, INC. LOCKHEEO MARTIN HAHFORD CORP. JOB HO. E61945/F3RUL5

FILE HO. Z475AAFI

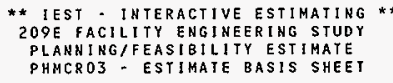

PAGE 4 OF 9

$14: 17: 07$

D. SITE ALLOCATIONS FACTORS

TEVLOPED AND PROVIDED BY FLUOR DANIEL HANFORD (FDH) FOR ESTIMATING USE.

TEO TO ALL COSTS TO LIOUIOATE GOVERHMENT FURNISHED SERVICES PROVTDED TO THE CORIISHED SERVICES RATE IS APPI

TOLTUUIDATE THE HANFORD GENERAL \& ADKINISTRATIVE COSTS

(3) HANFR STE GQA RATE OF $70 \%$ IS APPLIED TO ALL PURCHASED MATERIAL ANO $7.7 \%$ IO ALL PURCHASED SERICES TO LIQUIDAIE THE COST OF PROCUREMENT (INCLUDING RECIEVING).

FONH APPLIES THE ABOVE FACTORS TO ESTIMATED COSTS AS FOLLOWS

(1) FDH GFS IORA CH FACTOR: COMPOSITE FACIOR OF 30 OL\% HAS BEEN APPLIED TO TOTAL FDNH FIXED PRICE CONSTRUCTION

MANAGEMENT WHICH INCLUDES GOVERMHENT FURHISHED SERVICES (GFS) AND SITE G\&A/FEE.

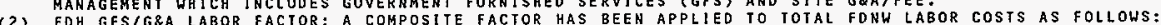
AE CCM COSTS = $33.04 \%$ FDNHS CONSTRUCTION LABOR $=28.37 \%$, FDNWS CONSTRUCTION MANAGEMENT LABOR $=33$.04\%, FDNH CONIRACI

FITE FACTOR OF $24.87 \%$ HAS BEEN APPLTED TO TOTAL FDNW MATERIAL COST WHICH INCLUDE A MPR OF $7 \%$ ARD MATERIAL G\&A/FEE OF $16.7 \%$

\section{ESCALATION}

ESCALATION PERCENTAGES HERE CALCULATED FROM THE JANUARY 1997 UPDATE OF THE ECONOMIC.ESCALATION PRICE CHANGE INDICES FOR DOE ESCALATION PERCENTAGES WERE CALCULATED FROM THE JANUARY TOST UPDATE OF THE ECONOMIC. ESCAL.

5. CONTINGENCY

A. DEFINITION OF CONTINGENCY AS PROVIDED BY DOE

"CONTIMGENCY COVERS COSTS THAT MAY RESULT FROM INCOMPLETE DESIGN, UNFORESEEN AND UNPREDICTABLE CONDITIONS, OR

PROCUREMENT AND CONSTRUCTION: AND THE COMPLEXITY AND UMCERTAINTIES OF THE COMPONENT PARTS OF IHE PROJECT. CONTINGENCY IS NOT YO BE USED TO AVOID MAKING AN ACCURATE ASSESSMEMT OF EXPECTEO COST" (OFFICE OF HASTE MANAGEMENT (EM-3O) COST AND SCHEDULE GUIDE.

B. COnTINGency allowance guidelines

IHE DOE GUIDELINE CONTINGENCY ALLOHANCE FOR A PLANNING/FEASIBILITY ESTIMAIE IS $20 \%$ TO $30 \%$,

C. METHODOLOCY

CONTIHGENCY IS EVALUATED AT THE LOHEST HORK BREAKDOWN STRUCTURE (WBS) LEVEL WITHIN THE COST ESTIMATE DETAILS. IT IS SUMHARIZEO AT UPPER HBS LEVELS AND REPORTED ON THE SUMMARY REPORTS

D. ANALYSIS

AN ASSESSMENT OF DESIGN MATUR!TY, WORK COMPLEXITY AND PROJECT UHCERTAINTIES HAS BEEN PERFORMED. AN EKPLAIHATION OF THIS ASSESSMENT AND CONTIGENCY RATES WHICH HAVE BEEH ADDED TO THE COST OF WORK ARE AS FOLLOHS:

H8S $31 X X X X$ CONSTRUCTION, ENGIHEERING AND PROJECT MAHAGEMENT - A $30 \%$ CONTINGENCY HAS BEEH APPLIED TO COVER COSTS LIKELY TO SURFACE THAT HAVE NOT BEEN ANTICIPATEO AT THIS EARLY STAGE OF PLANHING. TECHNIQUES TO BE UTILIZED FOR DECONTAMINATION, STABILLIZATION AND REMOVAL HILL NEED FURTHER STUDY AND MANY OF THE ACIIVITIES WILL BE SUBJECT TO CHANGE AT ANY TIME DURING THE WORK PROGRESS. 
ELUOR DANIEL NORTHWEST, INC. LOCXHEED MARTIN HANFORD CORP. JOB NO. E61945/F3RULS

FILE NO. 2475 AAF1
* IEST - INTERACTIVE ESTIMATING ** 209E FACILIIYY ENGINEERING STUDY PLANMING/FEASIBILITY ESTIMATE PHACR03 - ESTIMATE BASIS SHEET
PAGE 5 OF 9

DATE $06 / 16 / 97 \quad 14: 17: 07$

BY

6. REMARKS

MAJOR ASSUMPTIONS WHICH HAVE BEEN MADE IN THE PREPARATION OF THIS ESITMATE aRE AS FOLLOWS:

A.) COSTS FOR CLOSURE CERTIFICATIONS, WIPE SAMPLES, SAMPLE ARALYTICAL COSTS, CORE SAMPLES, SOIL SAMPLES, TECHNICAL PUBLICATIONS, VERIFICATION SAMPLING AND A DECOMMISIONING PLAN HAVE NOI BEEN ADDRESSED, AND IF THESE ARE REOUIRED, WILL WEED TO BE INCLUDED.

B.) LIMITED INFORMATION WAS AVAILABLE ANO GROSS ASSUMPTIONS HERE MADE.

C.) THE ESTIMATE ASSUMED THAT BURH-OUT WOULD NOT BE ENCOUNTERED.

O.) ENGINEERING AND PROJECT MANAGEMENT COSTS HERE FIGURED AS A PERCENTAGE OF CONSTRUCTIOH, SEE ROB REPORT. 
FLUOR DANIEL NORTHHEST, IHC.

LOCKHEED MARTIN HANFORD CORP.

JOB NO. E61945/F3RULS

FILE NO. 2475AAFI

\section{SORT}

SORT
CODE/HS S DESCRIPTION

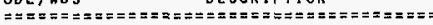

SDNW

310420 ALTERHATIVE 4 - STAGE 2

TOTAL FONW FLUOR DAHIEL NORTHHEST

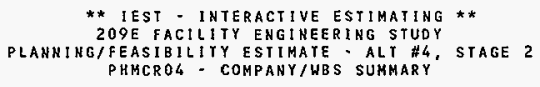

PEASIBILITY ESTIMATE ALTHA, STAGE 2
PHMCRO4 - COMPANY/WBS SUMMARY

PAGE 6 OF 9

$14: 05: 57$

BY DKH/RWO

\begin{tabular}{|c|c|c|c|c|c|c|c|c|}
\hline $\begin{array}{l}\text { ESTIMATE } \\
\text { SUBTOTAL } \\
=======\end{array}$ & $\begin{aligned} & E S C A \\
\% & == \pm=\end{aligned}$ & $\begin{array}{l}\text { ALATION } \\
\text { TOT AL } \\
====\equiv==\end{array}$ & $\begin{array}{c}\text { SUB } \\
\text { TOTAL } \\
===\equiv=\equiv==\end{array}$ & $\begin{aligned} & \operatorname{con} \tau \\
\% & \%==\end{aligned}$ & $\begin{array}{l}\text { NGENCY } \\
\text { TOTAL } \\
=====\equiv=\end{array}$ & $\begin{array}{c}\text { SUB } \\
\text { TOTAL } \\
=\approx===\equiv==\approx\end{array}$ & $\begin{array}{c}\text { SITE } \\
\text { ALLOCAT'M } \\
====\pi===\end{array}$ & $\begin{array}{c}\text { TOTAL } \\
\text { OOLLARS } \\
===\equiv= \pm==\equiv\end{array}$ \\
\hline $\begin{array}{l}52302 \\
52302\end{array}$ & $\begin{array}{l}0.00 \\
0.00\end{array}$ & $\begin{array}{l}0 \\
0\end{array}$ & $\begin{array}{l}52302 \\
52302\end{array}$ & $\begin{array}{l}30 \\
30\end{array}$ & $\begin{array}{l}15690 \\
15690\end{array}$ & $\begin{array}{l}67992 \\
67992\end{array}$ & $\begin{array}{l}21236 \\
21236\end{array}$ & $\begin{array}{l}89228 \\
89228\end{array}$ \\
\hline $\begin{array}{l}7644 \\
7644\end{array}$ & $\begin{array}{l}0.00 \\
0.00\end{array}$ & $\begin{array}{l}0 \\
0\end{array}$ & $\begin{array}{l}7644 \\
7644\end{array}$ & $\begin{array}{l}30 \\
30\end{array}$ & $\begin{array}{l}2293 \\
2293\end{array}$ & $\begin{array}{l}9937 \\
9937\end{array}$ & $\begin{array}{l}0 \\
0 .\end{array}$ & $\begin{array}{l}9937 \\
9937\end{array}$ \\
\hline 59,946 & & 0 & & 30 & & 77,929 & & 99,16 \\
\hline
\end{tabular}

L MHC

LOCKHEED MARTIN HANFORD CORP.

310420 ALIERHATIVE 4 . SIAGE 2 TOTAL LMHC LOCKHEED MARTIN HANEORD COR 
FLUOR DANIEL HORTHHEST, INC. LOCKHEED MARTIN HANFORD CORP. JOB NO. E61945/F3RULL

FILE NO. 2475AAF?

WBS DESCRIPIIOH

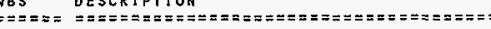

310420 alternative 4 - Stage 2

PROJECT TOTAL
* IEST * INTERACTIVE ESTIMATING **

PLANNING/FEASIBILITY ESTIMATE. ALT H4, STAGE 2

PHMCROS - CONSTRUCTION MANAGEMEMT/OTHER COST SUMMARY

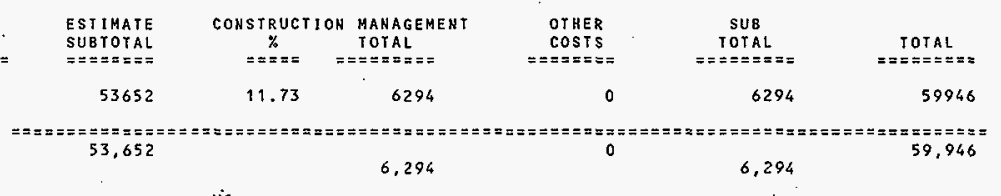


FLUOR DANIEL NORTHWEST, INC. LOCKHEED MARTIN HANFORD CORP. JO8 NO. E61945/F3RULS

FILE NO.: Z475AAF1

WBS DESCRIPIION

WBS DESCRIPIION

310420 ALTERNATIVE 4 - STAGE 2

PROJECT TOTAL
* IEST - INTERACTIVE ESTIMATING *

209E FACILITY ENGINEERING SIUDY
PLANMING/FEASIBILITY ESTIMATE. ALT \#4, STAGE PHMCROG - SITE ALLOCATIONS BY WBS
PAGE 8 OF

$15: 06: 59$

BY DKH/RHO

\section{ESTIMATE DYH FDH GFS/G\&A FOH MPR FDH GFS/G\&A FDH HPR/G\&A SITE ALLOC SUBTOTAL EQ.USAGE CONST.MGMT F.P./S.C. LABOR MATERIAL SUBTOTAL}

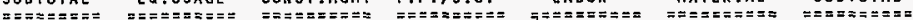
2033
2080

0

12011

21

16335

53,652

2,033

2,080

12,011

211 


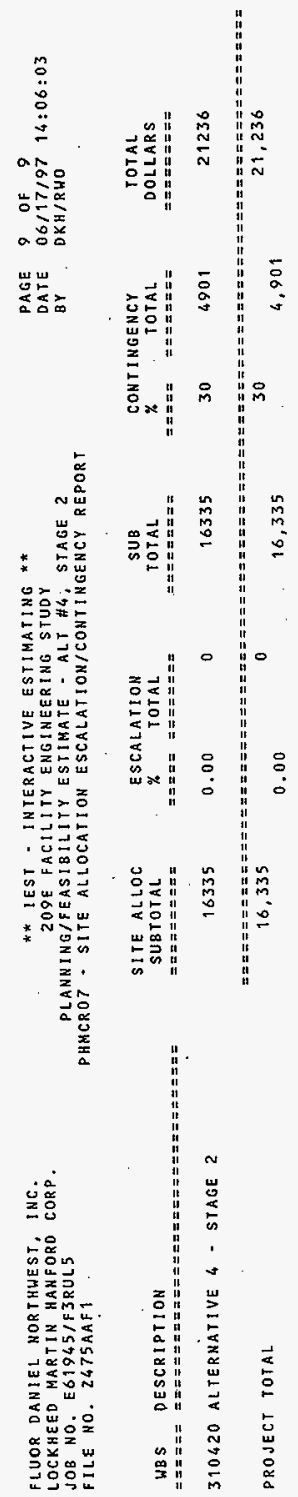



FLUOR DANIEL NORTHHEST, INC.
LOCKHEED MARTIN HAHFORO CORP. JOB NO. E61945/E3RUL5 FILE HO. Z475AAF 1

ACCOUNT

NUMBER DESCRIPTION ALTERNATIVE 4 - STAGE 2

310420 TECHNICAL SERVICES

310420.00

310420.0004200

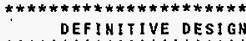
*

3104200005200 AT $25 \%$ OF CONSTRUCTION ENGIHEERING INSPECTION AT $15 \%$ OF CONSTRUCTION

310420.0005300 AT 15\% OF CONSTRUCTION PROJECT MAHAGEMENT AT $15 \%$ OF CONSTRUCTION

SUBTOTAL TECHNICAL SERVICES

TOTAL COSI CODE D0000 HBS 310420 (ESCALATION

$0.00 \%-\operatorname{CONTINGENCY~}$

* IEST - INTERACTIVE ESTIMATING * PLANNIMG/FEASIBILITY ESTIMATE - ALT \#4, STAGE 2 PHMCRO8 - ESTIMATE DETALL BY WBS, COST CODE

$1 \mathrm{~L} / \mathrm{S}$

8778

$1 \mathrm{L/S} \quad 88 \quad 5280$

$1 \mathrm{~L} / \mathrm{s} \quad 72 \quad 5256$

$$
293
$$

0

$293 . \ldots .314$

$\begin{array}{lll}\text { PAGE } & 1 \\ \text { DATE } & 06 / 17 / 97 & 14: 06: 05\end{array}$

BY DKH/RWO

COST EQUTP SUB: EQUIP- OH\&P TOTAL

CODE QUANTITY MANROURS LABOR USAGE MATERIAL CONTRÁCY MENT, (1)

310420.01 GENERAL REQUIRMENTS

310420.0105000 HPT TO MAN STEP-OFF PAD

SUBTOTAL GENERAL REQUIRMENTS

SHP $100.00 \%$

TOTAL COST CODE 81001

HBS 310420

$0.00 \%$ - CONIIHGEHCY $30.00 \%$

810 M
(MASK)
ENCY $30.00 \%)$

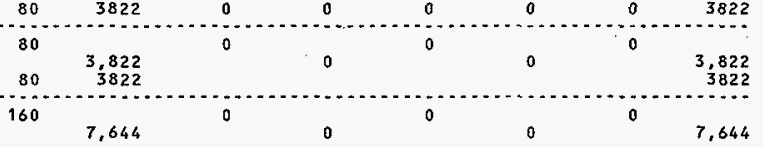

310420.02
310420.0205000 SI TEWORK

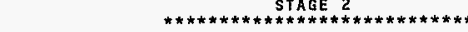

310420.0205002 REMOVE SUSPECTED CONTAMIN-

$810 \mathrm{M} \quad 0$

0

0

$0 \quad 5256$

810 H $11 / 5$

o

(1)

0

0

0

9,314

AIED PIPING, AIR RECIRCUL-

ATION SYSTEK, COOLING U. 

JOB NO. E61945/F3RULS

FILE NO. 2475AAF 1

ACCOUNT

NUMBER

FLUOR DANIEL NORTHWEST, INC, LOCKHEED MARTIN HANFORD CORP.

* IEst - inTeractive estimating *

209E FACILITY PLANHING/FEASIBILITY ESTIMATE ALT\#4, STAGE 2 PHMCR0 - - ESTIMATE DETAIL BY WBS, COST CODE DESCRIPYION

COST EQUIP

EQUIP SUB-

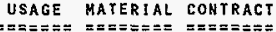

PAGE

DATE $06 / 17 / 97 \quad 14: 06: 00$

BY DKH/RUO

EQUIP- OH\&P TOTAL MENT I B \& I DOLLARS (unsk 6 EA 224 0 8,064 0

10
6,000 o
CONSUMABLES $3.20 \%$

GENERAL FOREMAN $7.00 \%$

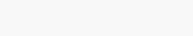
GENERAL REQUIREMENTS $15.00 \%$ SALES IAX $8.00 \%$ DH\&P (OM MARKUPS.ONLY)

TOTAL COST CODE 81002 COST CODE 810 CESCALATION

$0.00 \%$ - CONTINGEHCY $30.00 \%$

$\begin{array}{rrr}224 & 14,582 \\ 3064 & 258\end{array}$

$\begin{array}{rrr}31 & 1128 & 1284 \\ 71 & 2588 & 1128\end{array}$

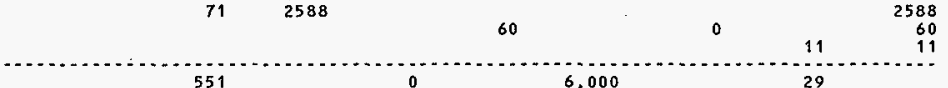

551

818

26,693

TOTAL. WBS 310420 ALTERNATIVE 4 - STAGE 2 .

$1,004 \quad 46,803$

818

6,000

0

29

53,651 
FLUOR DANIEL HORYHWEST, INC.

LOCXHEED MARTIN HAHFORD CORP

ACCOUN

NUMBER

DESCRIPTIOH

NCCOUNT

REPORT TOTAL

$\cos T$

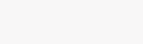

JOB NO. EGT945/F3RUL

* * IEST - INTERACTIVE ESTIMATIHG * 209E FACILITY ENGINEERING STUDY PLANNINOAFESIBILITY ESTIMATE. ALT \#4, STAGE

CODE QUAHTITY MAHHOURS LABOR EQUTP

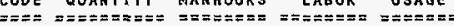

SUT -

SUB-

$\begin{array}{lll}\text { PAGE } & 3 & 3 \\ \text { DATE } & 06 / 17 / 97 & 14: 06: 06\end{array}$

DAY DKH/RUO

EQUIP. OHEP

MENT $======1$ \& I DOLLARS

REPORT TOTAL
46,803

\section{1,004}

\begin{abstract}
46,803
\end{abstract}


FLUOR DAHIEL NORTHWEST, JNC. LOCKHEED MARTIN HARFORO CDRP. JOB HO. E61945/F3RUL5

* TEST - INTERACTIVE ESTIMATING * 209E FACILITY ENGINEERING STUDY

ILE NO. Z475AAG1
PLANNIHG/FEASIBILITY ESTIMATE - ALT \#4, STAGE 3

PHMCRO1. PROJECT COST SUMMARY
PAGE 1 OF 9

DATE $06 / 17 / 97 \quad 14: 18: 14$

BY DKH/RWO

\section{SORT DESCRIPTION \\ FDRW FLUOR DANIEL NORIHHEST \\ SUBTOTAL \\ SITE SITE ALLOCATIONS \\ PROJECT TOTAL}

SORP $===\quad$ DESCRTPYION

LMHC LOCKHEED MARTIN HANFORD CORP.

\begin{tabular}{|c|c|c|c|}
\hline $\begin{array}{l}\text { ESCALATED } \\
\text { TOTAL COST } \\
=====\approx===\end{array}$ & $==\pi=\pi$. & $\begin{array}{l}\text { UGEUCY } \\
\text { YOTAL } \\
===\approx \approx=====\end{array}$ & $\begin{array}{c}\text { TOIAL } \\
\text { DOLLLARS } \\
====\equiv==\equiv==\end{array}$ \\
\hline 170,802 & 30 & 51,241 & 222,043 \\
\hline 44,836 & 30 & 13,451 & 58,287 \\
\hline
\end{tabular}

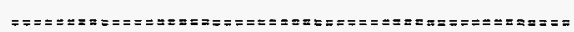

$\begin{array}{llll}215.638 & 30 & 64.692 & 280,330 \\ 57.506 & 30 & 17,252 & 74,758\end{array}$

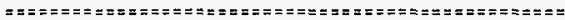

$\begin{array}{llll}273,144 & 30 & 81,944 & 355,088\end{array}$

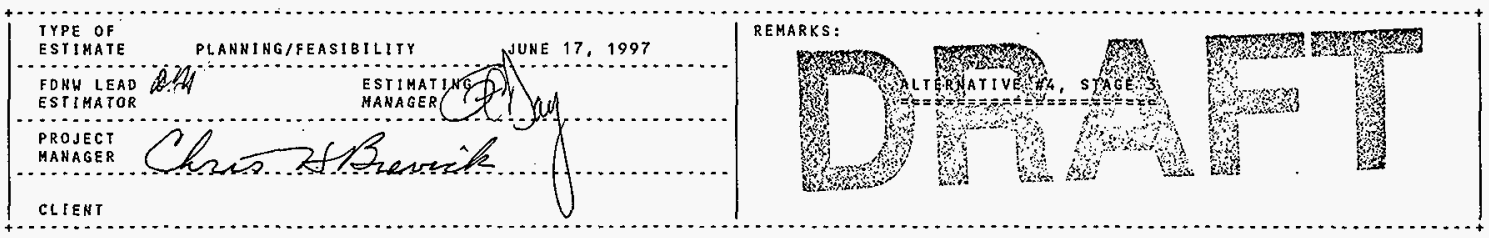


FLUOR DAHIEL NORTHUEST, INC.

LOCKHEED MARTIN HANFORD CORP.

FILE NO Z475AAG1

HBS DESCRIPTION

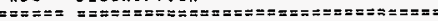

310430 ALTERNATIVE 4 - STAGE 3

PROJECT TOTAL
** IEST = IHTERACTIVE ESTIMATING * PLANRING/FEASIBILITY ESTIMATE - ALT \#4, STAGE 3 PLANRING/FEASIBILITY ESTIMATE - ALT \#4, STAGE 3
PHHCRO2 - WORK BREAKDOUN STRUCTURE (WBS) SUMMARY
PAGE 2 OF 9

DATE $06 / 17 / 97 \quad 14: 18: 17$

OKH $/$ RHO

\begin{tabular}{|c|c|c|c|c|c|c|c|c|}
\hline $\begin{array}{l}\text { ESTIMATE' } \\
\text { SUB TOTAL } \\
========\end{array}$ & $\begin{array}{l}\text { ESCA } \\
\%===\end{array}$ & $\begin{array}{l}\text { LATION } \\
\text { TOTAL } \\
====\pi==\end{array}$ & $\begin{array}{c}\text { SUB } \\
T \text { TOTAL } \\
=======\end{array}$ & $=\stackrel{\operatorname{con} x}{\%}$ & $\begin{array}{l}1 \text { NGENCY } \\
\text { TOTAL } \\
====x===\end{array}$ & $\begin{array}{c}\text { SUB } \\
\text { TOTAL } \\
=====\Rightarrow=\neq\end{array}$ & $\begin{array}{c}\text { SITE } \\
\text { ALLOCAT' } \\
=========\end{array}$ & $\begin{array}{c}\text { TOTAL. } \\
\text { DOLLARS } \\
== \pm== \pm==\end{array}$ \\
\hline 215638 & 0.00 & 0 & 215638 & 30 & 64692 & 280330 & 74758 & 355088 \\
\hline 15,63 & & $\theta$ & & 30 & & 280,330 & & 088 \\
\hline
\end{tabular}


FLUOR OANIEL NORTHWEST, INC. LOCKHEED MARTIN HANFORD CORP. JOB NO. E61945/F3RUL5

FILE NO, Z475AAG1
* IEST - INTERACTIVE ESTIMATING *
209E FACILLTY ENGINEERING STUD
PHMCROZ . ESTIMATE BASIS SHEET

PAGE 3 OF 9

$14: 17: 07$

BY DKH/RWO

1. ESTIMATE PURPOSE

THIS ESTIMATE WILL BE USED FOR A SCOPING STUDY.

2. ESTIMATE TECHNICAL BASIS

A. THIS ESTIMATE HAS BEEN PREPARED FOR LOCKHEED MARTIN HANGORD CORP. AS REQUESTED BY FDNW PROJECT HANAGER

B. A ROUGH DESCRIPIION OF THE SCOPE OF HORK MAY BE FOUND IN THE OUTLINE OF ALTERNATIVES, AN UNDATED AMO

C. THE FOLLOWING CONSTRAIUTS AND/OR SPECIAL CONDITIOHS EXIST: ALL HORX DONE IN THE CRITICAL ASSEMBLY ROOM AND IN THE MIXING ROOH HILL REQUIRE FOLLOWING THE APPROPRIATE RADIATIOH HORK PROCEDURE.

3. ESTIMATE METHODOLOGY

A MAHLOADING TECHMIOUE HAS USED TO COME UP WITH THE LABOR HOURS WHEN MECESSARY DUE TO THE UHDEFINED SCOPE.

A MANLOATNG TECHNICUE WAS USED TO COME OP HITH THE LABOR YOURS WHEN WECESSARY DUE TO THE UNDEFINED SCOPE. CONSTRUCTION LABOR, MATERIAL AND EQUIPMEN UNITS HAVE BEEN ESTIMATED BASED UPOH
COMMERCIAL ESTIMATING RESOURCES, PUBLISHED ESTIMATING MANUALS AND R, S. MEANS

B. DIRECT COST FACTORS

(1) SALES TAX HAS BEEN APPLIED TO ALL MATERIALS AND EQUIPMENT PURCHASES AT $8 \%$.

(2) NO WAREHOUSING COSTS ARE SHOWN SINCE YHEY ARE CONSIDERED TO BE INCLUDED IN IHE MATERIAL PROCUREMEHY RATE (MPR)

(3) AN ESTIMATING FACTOR OF $15 \%$ HAS BEEN APPLIED TO DIRECT CRAFT LABOR FOR GENERAL REQUIREMENTS AHD $23.58 \%$ FOR TECHNICAL SERVICES

(4) CONSUMABLES ARE ESTIMATED AT $3.2 \%$ OF DIRECT CRAFT LABOR COSTS

(5) SPECIAL HORK PROCEDURE (SHP) FACTORS ARE APPLIED AGAINST DIRECT LABOR FOR ACTUAL TIME LOST DUE TO IHE PERSONNEL PROTECTIVE EQUIPMENT AND PROCEDURES. THE RATES. WHICH HAVE BEEN APPLIED ARE AS FOLLOUS: PROTECTIVE CLOTHING FACTOR HAS BEEN APPLIED AT $15 \%$

MASK WORK AT $85 \%$ PLUS $15 \%$ FOR PROTECTIVE CLOTHIHG.

CONTAMINATION RESTRICTIONS ASSOCIATED WITH RADIATION DOSE LIMITS (BURNOUT) HAVE NOT BEEN CALCULAIED AS IT IS NOT BELIEVE TO BE A CONCERN FOR THESE ACTIVITIES.

(6) PREMIUM PAY

OVERTIME REQUIREMENTS ANO SHIFT OIFFEREMTIAL PAY FOR CRAFT LABOR WILL NOT BE REQUIRED FOR THIS HORK, AS STRAIGHT TIME, 40 HOUR WEEKS ARE DEEMED ADEQUATE TO ACCOMPLISH THIS WORK.

(7) GENERAL FOREHAN FACTOR OF $7 \%$ HA

(8) A FACTOR OF 10\% HAS BEEN APPLIED TO DIRECT CRAFT LABOR TO ALLOW FOR USAGE OF GOVERNMENT OWNED EQUIPMENT COHTROLLED BY DYNCORP.

C. RATES

(1) FLUOR DANIEL NORTHHEST LABOR RATES ARE BASEO UPON THE FLUOR OA.INEL FEDERAL OPERATIONS (FEDFO). DISCLOSURE STATEMENT AND APPROVED PROVISIONAL BILLING RATES. FOR ESTIMATING PURPOSES, AVERAGE RATES BY OPERATIONS CODE HAVE BEEN DEVELOPED BASED UPON RECEUT

(2) FLUOR DANIEL NORTHWEST SERVICES (CONSTRUCTION CRAFT LABOR) RATES ARE THOSE LISTED IN APPENDIX A TO THE HANFORD SITE

(3) FDH \& PHMC SUBCONTRACTOR STANDARD LABOR RATES ARE THOSE LISTEO IN THE FIHANCIAL DATA SYSTEM (FDS) FDST $321 R$ REPORT ORGANIZATION RATES PLUS ADDERS. 
FLUOR DANIEL NORTHUEST, INC. LOCKHEED MARTIN HANFORD CORP. JOB NO. $861945 / F 3 R U L S$

FILE NO. Z475AAG

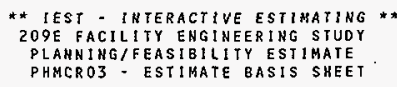

$\begin{array}{ll}\text { PAEE } & 4 \text { OF } 9 \\ \text { DATE } & \text { O6/16/97 } \\ \text { BY } & \text { DKH/RWO }\end{array}$

D. SITE ALLOCATIONS FACTORS

TITE ALIOCATION FACTORS ARE DEVELOPED AHD PROVIDED BY FLUOR DANIEL HANFORD (FDH) FOR ESIIMATING USE.

(1) GOVERNMENT FURHISHED SERVICES RATE IS APPLIED TO ALL COSTS TO LIOUIDATE GOVERNMENT FURNISHED SERVICES PROVIDED TO THE ENTERPRISE COMPANIES: $14 \%$ FOR FDNU, $10 \%$ FOR FONWS (CONSTRUCTION).

(2) HANFORD SITE GQA RATE OF $16.7 \%$ IS APPLIED TO ALL COSTS TO LIOUIDATE THE HANEORD GENERAL \& ADMINISTRATIVE COSTS.

(3) HANFORD SITE MPR QATE OF $7.0 \%$ IS APPLIED TO ALL PURCHASED MATERIAL AHD $7.7 \%$ TO ALL PURCHASED SERICES TO LIOUIDATE THE COST OF PROCUREMENI (INCLUDING RECIEVING).

FDYW APPLIES THE ABOVE FACTORS IO ESTIMATED COSTS AS FOLLOWS

(1) FDH GFS/G\&A CM FACTOR: A COMPOSITE FACTOR O.F $33.04 \%$ HAS BEEN APPLIED TO TOTAL FDNH FIXED PRICE CONSTRUCTION

(1) FDH GFSIG\&A CM FACTOR:

(2) FDH GFSIG\&A LABOR FACTOR: AOMPOSITE FACTOR HAS BEEN APPLIED TO TOTAL FDNW LABOR COSTS. AS FOLLOHS: AE $/$ CM COSTS $=33.04 \%$, FDHHS CONSTRUCTION LA8OR $=28.37 \%$, FDNWS CONSTRUCTION MANAGEMENT LABOR $33.04 \%$, FDNW CONTRACT MAMAGEMENT AND ADMIHI STRATION $=33.04 \%$

(3) FDH MPR /G\&A MATERIAL FACTOR: A COMPOSITE

A MPR OF $7 \%$ AND MATERIAL G\&A/FEE OF $16.7 \%$

4. ESCALATIOH

ESCALATION PERCENIAGES HERE CALCULATED FROM THE JAHUARY 1997 UPDATE OF THE ECONOMIC ESCALATION PRICE CHAHGE INDICES FOR DOE CONSTRUCTION PROJECTS AS PUBLISHED BY THE "OFFICE OF INFRASTRUCTURE ACQUISITION" FM-5O.

5. CONTINGENCY

A. DEFINITION OF COHTINGENCY AS PROVIDED BY DOE.

"CONTINGENCY COVERS COSTS THAT MAY RESULT FROM INCOMPLETE DESIGN, UNFORESEEN AHD UHPREDICTABLE CONDITIONS, OR UNCERTAINTIES WITHIH THE DEFINED PROJECT SCOPE. THE AMOUNT OF CONTINGENCY HILL DEPEND OH THE STATUS OF DESIGN, PROCUREMENT, AND CONSTRUCTION; AND THE COMPLEXITY AND UNCERTAINTIES OF THE COMPONENT PARTS OF THE PROSECT. CONTIHGENCY IS NOT TO BE USED TO AVOID. MAKING AN ACCURATE ASSESSMENT OF EXPECTED COST" (OFFICE OF WASTE MANAGEMENT (EM-3O) COST AND SCHEDULE GUIDE.

B. CONTINGENCY ALLOWAHCE GUIDELIHES

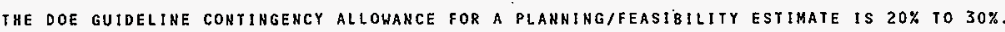

C. METHODOLOGY

CONTHGENCY IS EVALUATED AT THE LOWEST HORK BREAKDOWN STRUCTURE (WBS) LEVEL WITHIN THE COST ESTIMATE DETAILS. IT IS SUHMARIZED AT UPPER HBS LEVELS AND REPORTED ON THE SUMMARY REPORIS

D. AHALYSIS

AH ASSESSMENT OF DESIGH MATURITY, WORK COMPLEXITY AND PROJECT UNCERTAINTIES HAS BEEN PERFORMED. AH EXPLAIHATION OE THIS AT ASSESSHENT OF DESIGH MATURITY, WORK COMPLEXITY ANO PROJECT UNCERTATNTYES HAS 8EEN PERFOR

H8S $31 \times X X X$ CONSTRUCTIOH, ENGINEERING AND PROJECT MANAGEMENT - A $30 \%$ CONTINGENCY HAS BEEN APPLIED TO COVER COSTS LIKELY TO SUREACE THAT HAVE HOT BEEN ANTICIPATED AT THIS EARLY STAGE OF PLANAING. TECHNIOUES TO BE UTILIZED FOR DECONTAMINATION, STAB THE WORK PROGRESS. 
FLUOR DANIEL NORTHEST, INC.

LOCKHEED MARTIN HAMFORD CORP.

JOB NO. E61945/F3RUL5

* IEST - INTERACTIVE ESTIMATING *

209E FACILITY ENGINEERIHG STUDY

PLANNING/FEASIBILLTY ESTIMATE
PAGE 5 OF 9

DATE $06 / 16 / 97 \quad 14: 17: 07$

6. REMARKS

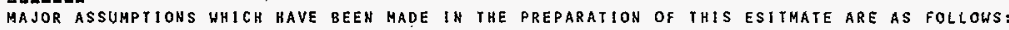

A.) COSTS FOR CLOSURE CERTIFICATIONS, WIPE SAMPLES, SAMPLE ANALYIICAL COSTS, CORE SAMPLES, SOIL SAMPLES, IECHNICAL PUBLICATJONS, VERIFICATION SAMPLING ANO A DECOMMISIONING PLAN HAVE NOT BEEN ADDRESSED, AMO IF THESE ARE REQUIRED, HILL NEED TO BE INCLUOED.

B.) LIMITED INFORMATIOH WAS AVAILABLE AND GROSS ASSUMPTIONS WERE MABE.

O.) ENGINEERING AND PROJECT MANAGEMEHT COSTS WERE FIGURED AS A PERCENTAGE OF CONSTRUCTION, SEE ROB REPORT. 
FLUOR DAHIEL NORTHWEST, INC.

LOCKHEED MARTIN HAHFORD CORP.

JOB NO. E61945/F3RUL5

FILE HO. Z475AAG1

SORT

CODE/HBS DESCRIPTION

Deription

FONW FLUOR DANIEL NORTHWEST

310430 ALTERNATIVE 4 - SFAGE 3 TOTAL FDNU FLUOR DANIEL NORTHWEST

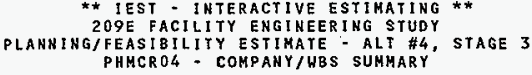

PLANNING/FEASIBILITY ESTIMATE - ALT \#4, STAGE 3
PHMCRO4 COMPANY/WBS SUMMARY

PAGE 6 OF 9

DAIE $06 / 17 / 97 \quad 14: 18: 20$

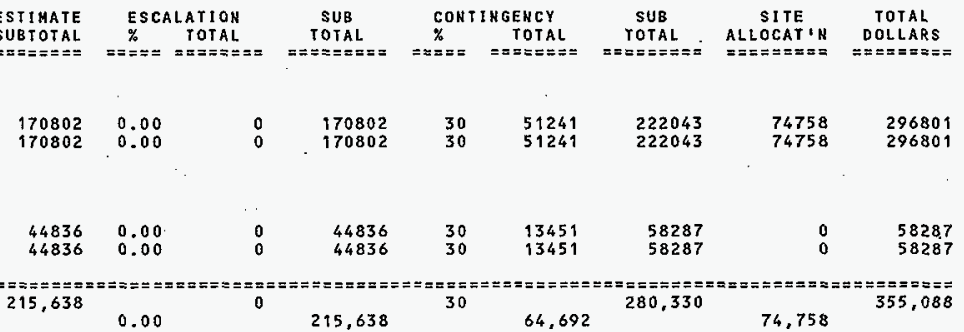


FLUOR DAHIEL NORTHHEST, IHC.

LOCKHEED MARTIH HANFORD CORP.

JOB NO. EOTS4STFBRULS

FILE HO. 2475AAG

WBS DESCRIPTION

W

310430 ALTERHATIVE 4 - STAGE 3

PROJECT TOTAL
** IEST - INTERACTIVE ESTIMATING *

209E FACILI IY.ENGINEERIHG STUDY

PHMCRO5 - COHSTRUCTION MANAGEMENT/OTHER COSY SUMMARY

\begin{tabular}{|c|c|c|c|c|c|}
\hline $\begin{array}{l}\text { EST IMATE } \\
\text { SUB TOTAL. } \\
====== \pm=\end{array}$ & 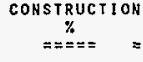 & $\begin{array}{l}\text { MANAGEMENT } \\
\text { TOTAL } \\
z=======\end{array}$ & $==\begin{array}{c}\text { OTHER } \\
\text { COSTS } \\
======\end{array}$ & $\begin{array}{c}\text { SUB } \\
\text { TOTAL } \\
====\equiv===\end{array}$ & $=====\equiv=\equiv=$ \\
\hline 196381 & 9.81 & 19257 & 0 & 19257 & 215638 \\
\hline
\end{tabular}

DATE

DAY OKH $/$ RHO
PAGE 7 OF 97 
FLUOR DANIEL HORTHUEST, INC.

LOCKHEED MARTIN HANFORO CORP.

JOB NO. E6I945/F3RULS

FILE NO. Z475AAG1

WBS DESCRIPTIOH

(1)

310430 ALTERHATIVE 4 - STAGE

PROJECT TOTAL
* * IEST - INTERACT IVE ESTIHATING * *

209E FACILITY ENGINEERING STUDY

PLAHIMG/FEASIBILITY ESIIMATE - ALI \#4, STAGE 3 PHMCROG - SITE ALLOCATIONS BY WBS
PAGE 8 OF 9

DATE $06 / 17 / 97 \quad 14: 18: 41$

BY DKH RWO

ESTIMATE DYH FOH GFS/G\&A FOH MPR FDH GFS/G\&A FDH MPR/G\&A SITE ALLOC

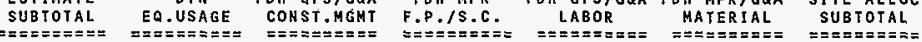
196381
7324
6363
$0 \quad 43370$
449
57506

196,381

7,324

6,363

43,370

449 
FLUOR DANIEL NORTHUEST, INC.

LOCKHEED MARTIN HAHFORD CORP.

JOB HO. E61945/F3RUL5

FILE NO. Z475AAG1

HBS DESCRIPTIOH

WBS

310430 ALTERHATIVE 4 - STAGE 3

PROJECT TOTAL
* * IEST * INTERACTIVE ESTIMATIHG * *

209E FACILITY ENGINEERING STUD

PLANHING/FEASIBILITY ESTIMATE - ALY \#4, STAGE 3
PAGE 9 OF 9

DATE $06 / 17 / 97$

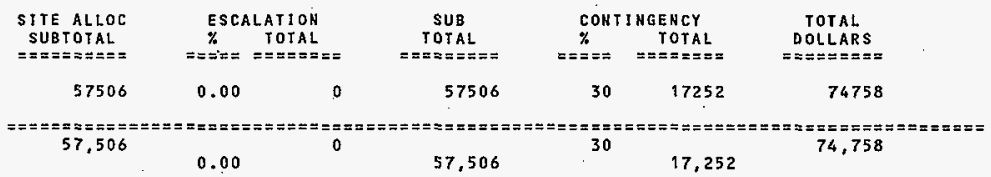


FLUOR DAHIEL NORTHHEST, INC. LOCKHEED MART IN HANFORD CORP. JOE NO. E61945/F3RUL5

FILE NO. 2475AAG

ACCOUNT
HUMBER
DESCRIPTIOH

ALTERHATIVE 4 - STAGE 3

310430

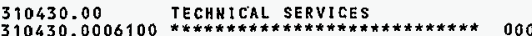

DEFINITIVE DESIGN

310430.0006200 AT $25 \%$ of CONSTRUCrION

ENGINEERING/ INSPECTION

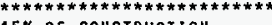

AT $15 \%$ OF CONSIRUCTION

PROJECT MANAGEMEH

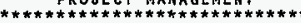

AT $15 \%$ OF CONSTRUCTION

SUBTOTAL TECHNICAL SERVICES

TOTA

$\operatorname{COST}$ CODE DO000

WBS 310430

CESCALATION $0,00 \%$ - CONTINGENCY

* IEST - IMTERACTIVE estIMATINg *

209E FACILITY ENGINEERIHG STUDY

PLANNING/FEASIBILITY ESTINATE ING STUDY STAGE PHMCROB - ESTIMATE DETAIL BY WBS, COST CODE

\section{COST.}

000

$1 \mathrm{~L} / \mathrm{s}$

318

19080

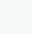

$1 \mathrm{~L} / \mathrm{S} \quad 261 \quad 19053 \quad 0$
PAGE

DATE $06 / 17 / 97 \quad 14: 18: 28$

DKH $/$ RHO CODE QUANTITY MANHOURS LABOR USAGE MATERIAL CONTRACT MEHT, /B \& I DOLLARS

SUB-

SUB - EQUIP

EQUIP. OH\&P TOTAL CODE QUANTITY MANHOURS

310430.01 GENERAL REQUIRHENTS BURIAL COSIS FOR 40 DRUMS OF 81 LLH CONCREYE DEBR IS AT $\$ 15.25 / C F$

SUBTOTAL GENERAL REQUTRMEHTS

TOTAL COST CODE 81001 $0.00 \%$ - CONTINGENCY $30.00 \%$

31746

0

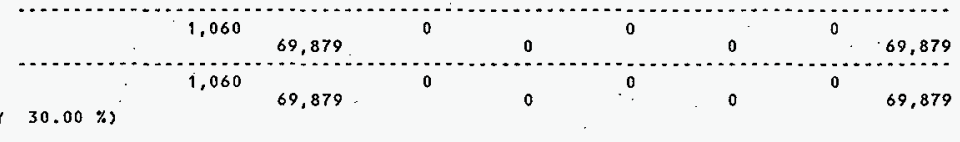

$\begin{array}{lllllllll}40 \mathrm{EA} & 0 & 0 & 0 & 0 & 4270 & 0 & 0 & 4270\end{array}$

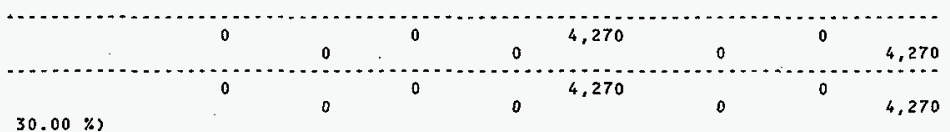

3१0430.01 GENERAL REQUIRMENTS

310430.0106000 HPT TO MAN STEP-OFF PAD

SUBTOTAL GEHERAL REQUIRMENTS

SHP $100.00 \%$

TOTAL COST CODE 81001

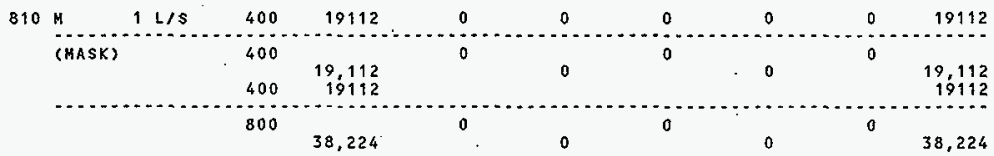


FLUOR DAHIEL NORTHWEST, IHC. LOCKHEED MARTIH HANFORÓ CORP. JOB NO. E61945/F3RUL FILE NO, 2475AAG 1

\section{ACCOUNT}

HUHBER DESCRIPTION

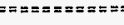

\section{CESCALATIOH}

* IEST - INTERACTIVE ESTIMATING 209E FACILITY ENGINEERING STUDY PLANHING/FEASIBILITY ESTIMATE - ALT \#4, STAGE 3
PHMCROS. ESTIMATE DETAIL BY HBS, COST CODE

COST EQUIP

$\cos 1$

QUAHTITY

MANROURS $30.00 \%$
PAGE
DATE
BY
BY
DKH/RHO

LABOR SUB-

MATERIAL CONTRACT

EQUIP - OH\&P TOTAL MENT IB \& I DOLLARS

310430.02 SITEHORK

310430.0206000

\section{STAGE}

REMOVE ALL REMALNIHG

EQUIPMENT

310430.0206022 BURIAL BOX ALLOHANCE

VEHTILATION SYSTEM - DUCTS :

VENTILATION SYST
FILTERS AND FANS

310430.0206042
310430.0206060 TERS AND FANS SEAL PIPING BETWEEN CAR AND

MIX ROOM

310430.0206080 SCABBLE/BUSH HA
WALLS AND FLOOR
310430.0206082 SURVEY

310430.0206082

310430.0206090 SCABBLE/BUSH HAMMER COHCRETE HALLS AHD FLOOR SECOND TIME IN HOT AREAS ONLY

310430.0206092 SURVEY

310430.0206100 SCABBLE/8USH HAMMER CONCRETE WALLS AND FLOOR THIRD TIME IN REMAIHING HOT AREAS.

310430.0206102 SURVEY 55 GAL W/LINER 310430.0206110 DRUM COSTS, 55 GAL W/LTER ISOLATE TANKS TK-109, 110

SUBTOTAL SITEWORK

CONSUMABLES $3.20 \%$

GEMERAL FOREMAN $7.00 \%$ GENERAL REQUIREMENTS $15.00 \%$ SALES TAX $8.00 \%$

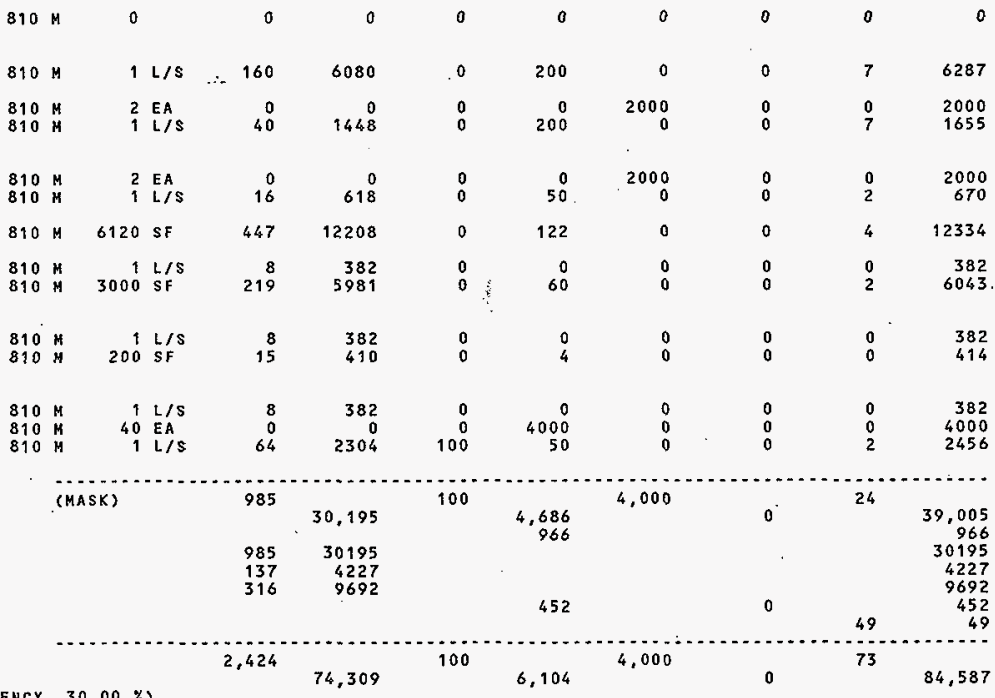

TOTAL

COST CODE 81002

WBS 310430

$0.00 \%$ - CONTINGENCY $30.00 \%$

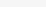


FLUOR DANIEL NORTHHEST, INC.

LOCXHEED MARTIH HAHFORD CORP.

JOB NO. E61945/F3RULS

* TEST - INTERACTIVE ESTIMATING * *

209E FACILITY ENGINEERING STUDY PLANNING/FEASIBILITY ESTIMATE AALT \#4, STAGE 3
PHMCROB . ESTIMATE DETAIL BY WBS, COST CODE

\section{$\cos T$}

ACCOUN

DESCRIPTION

COSTE QUANTIYY MAHHOURS LQU⿴囗十

NUHBER

SUB- EQUTP- OH\&P TOTAL
USAGE MATERIAL CONTRACT

NUMBER

TOTAL HBS 310430 ALTERHATIVE 4 - STAGE 3

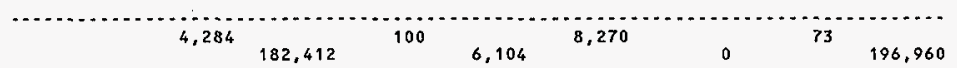




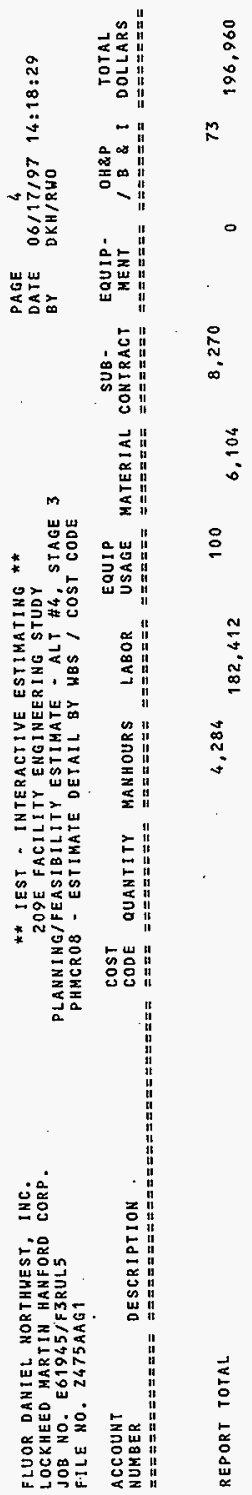


HNF-SD-WM-ES-411, Rev. 0

\title{
APPENDIX B
}

\author{
Schedules
}


HNF-SD-WM-ES-411, Rev. 0

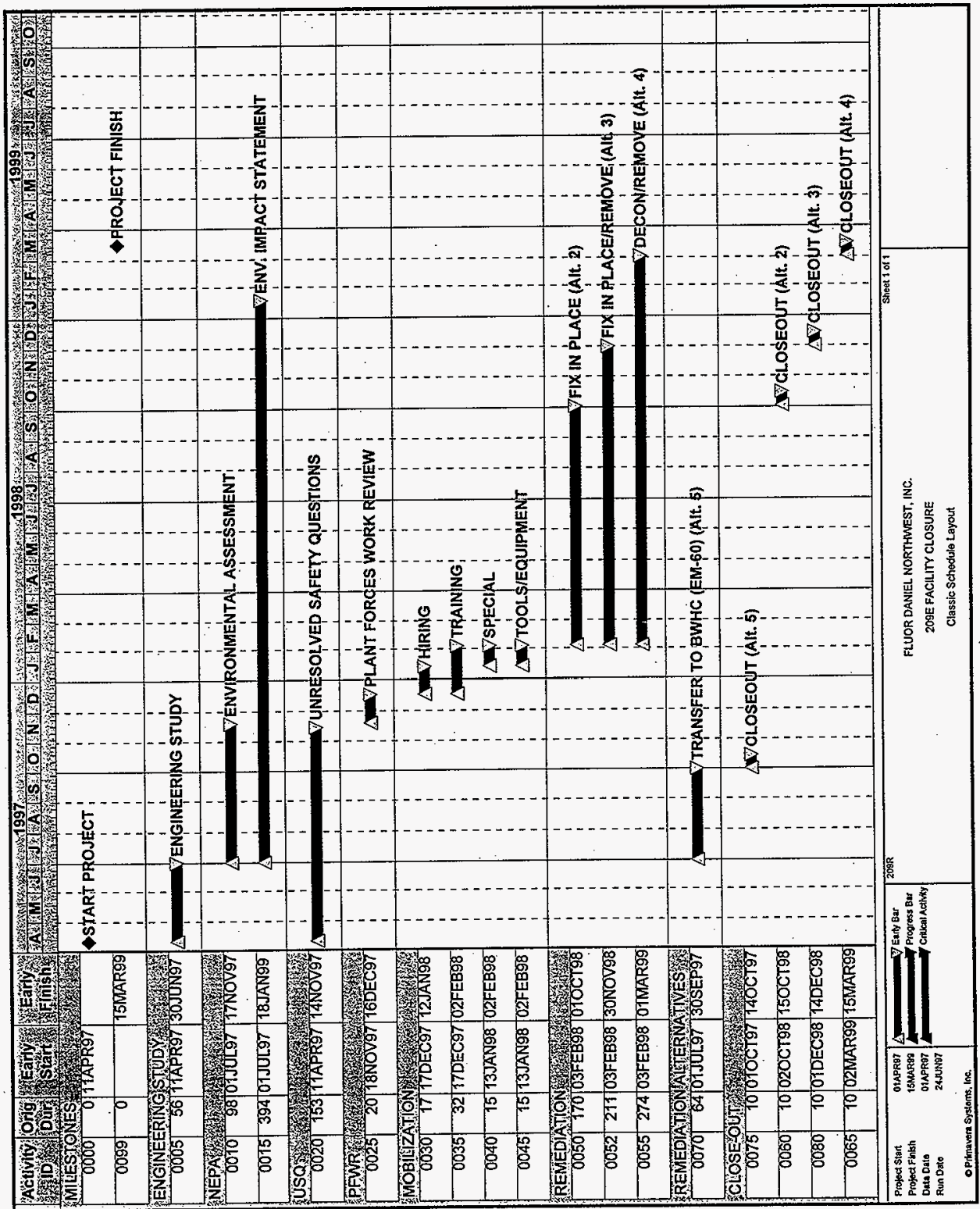


HNF-SD-WM-ES-411, Rev. 0

\section{APPENDIX C}

\section{Equipment List}

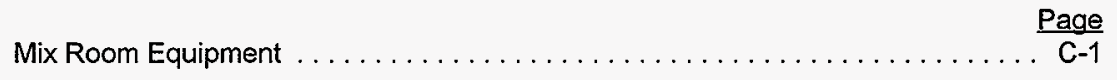

Critical Assembly Room Equipment $\ldots \ldots \ldots \ldots \ldots \ldots \ldots \ldots \ldots \ldots \ldots$ C-2 


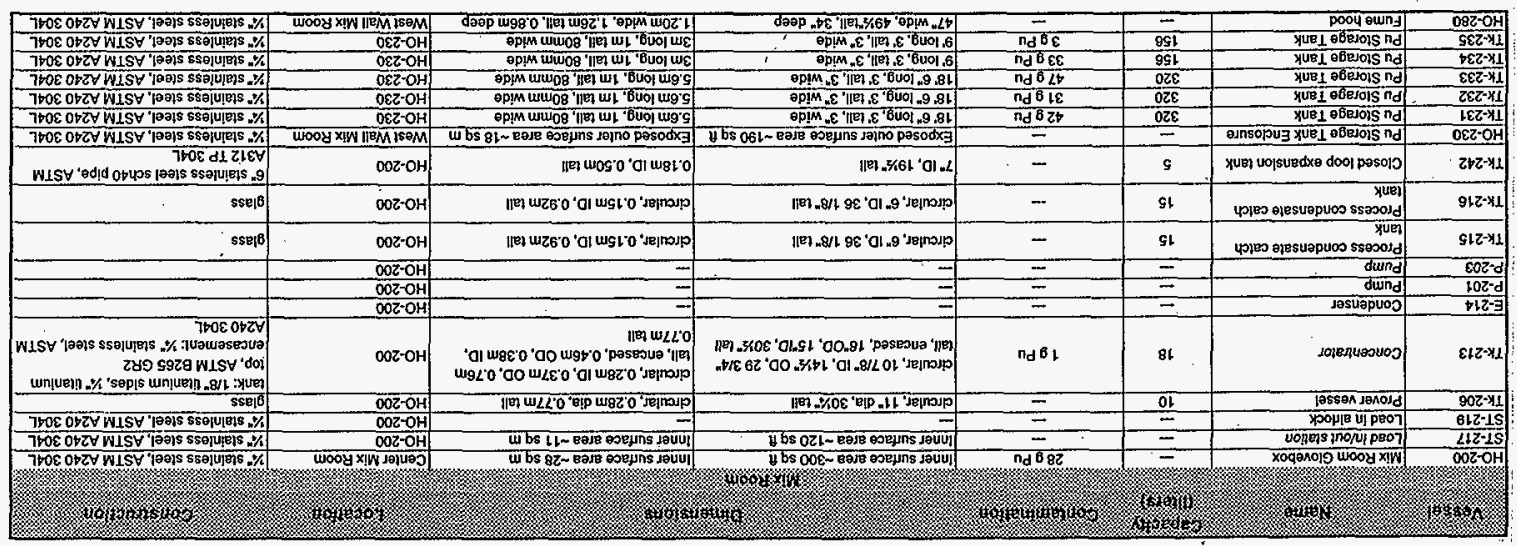




\begin{tabular}{|c|c|c|c|c|c|c|c|}
\hline 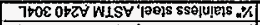 & $091-\times 1$ & 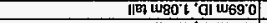 & $\| B=\angle B E Z b^{\circ} 01 . \angle Z$ & nd $b_{p}$ & oot & 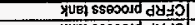 & $\frac{29 l-x_{1}}{101-41}$ \\
\hline 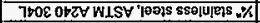 & $095-x \mid 1$ & IIE? WBO $L^{\circ}$ OII WgE & 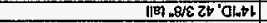 & $\ln B$ & OLt & 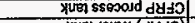 & $19 k-1 \mid$ |] \\
\hline 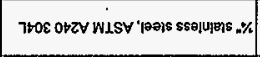 & ISeM '\&VO de!UeJ & 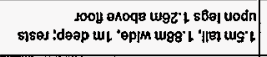 & 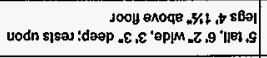 & - & - & 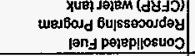 & $09 V-x_{1}$ \\
\hline 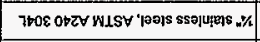 & 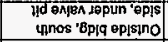 & 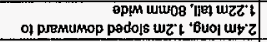 & 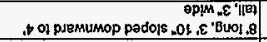 & umouyun Aqpueno & $0 z z$ & प्रUE⿱ dn-ppou ejsem & 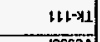 \\
\hline 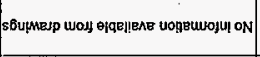 & SVES & 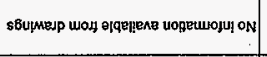 & 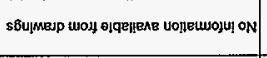 & $n_{d} 6 z$ & - & - & 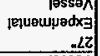 \\
\hline 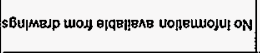 & SVBd & 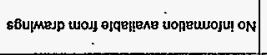 & 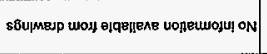 & nd $6 L Z$ & - & - & 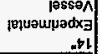 \\
\hline 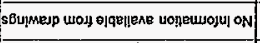 & 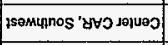 & 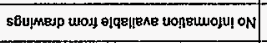 & 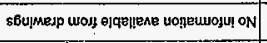 & - & - & 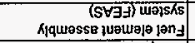 & $80 b^{-x} \mathbf{1}$ \\
\hline 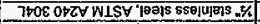 & ZYOIEMILOM & 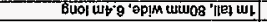 & 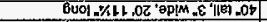 & ndB $B z L$ & $00 t$ & xuej $x \mid u$ dung & $901-31$ \\
\hline 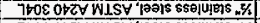 & प्रVO IFEM ISOM & 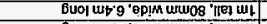 & 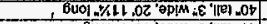 & $n_{9}^{8} 8 \varepsilon$ & oot & 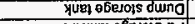 & $90 \mathrm{~L}-\mathrm{X}_{1}$ \\
\hline 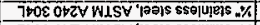 & ZVD IIEM पमON & 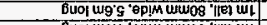 & 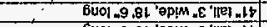 & $\mathrm{nd}^{\mathrm{B} G l}$ & 062 & 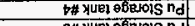 & $\$ 0 t-x_{1}$ \\
\hline 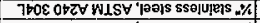 & УVD IIPM पNON & 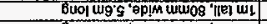 & 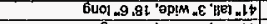 & $n_{d} B_{8}$ & 062 & E\# पue ebesols nd & $801-x \mid 1$ \\
\hline 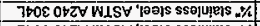 & $8 \forall \supset \| 2 M$ पLON & 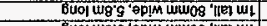 & 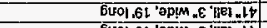 & - & $08 \varepsilon$ & Z\#\# Yuer abejols n & $201-71$ \\
\hline 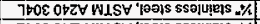 & 8VO IIBM पLON & Buol W & 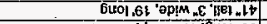 & Ad 6 L & OEE & If yue abzlois alesuapuog & $101-x \mid 11$ \\
\hline 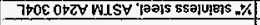 & OrLOH & 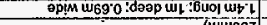 & 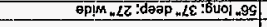 & - & - & 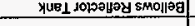 & $2+1-x_{1}$ \\
\hline 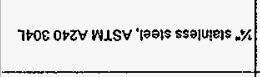 & $2+1-81$ & 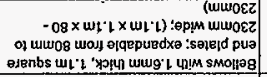 & 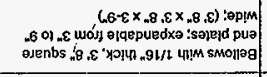 & $n_{d} B 96$ & शqueven & 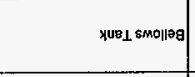 & $1+1-x_{1}$ \\
\hline 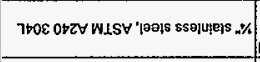 & 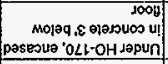 & 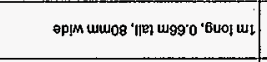 & 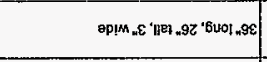 & $\begin{array}{l}\text { uMouxun } \\
\text { Ampuenb 'pəunssy }\end{array}$ & ot & 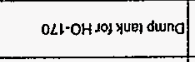 & OIL-11! \\
\hline 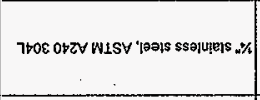 & 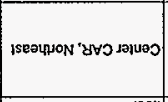 & \|le\} wg'b 'Odenbs ub'z & Ile? .St "ejents , 8 & 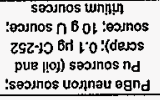 & - & $z \#$ pooH Riqueass $\forall$ & $\mathrm{OLLOH}$ \\
\hline 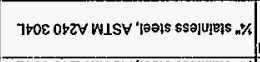 & 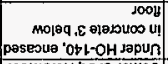 & 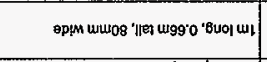 & 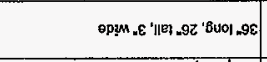 & umouyun Anueno & $0 \rightarrow$ & 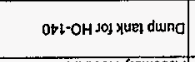 & $60|-3| 1$ \\
\hline \multirow[t]{3}{*}{7 Foe obEY WIS } & 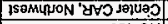 & 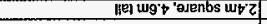 & liej, st ojenbs, 8 & - & $\rightarrow$ & I\# poOH Kquassy & $\mathrm{OBL}-\mathrm{OH}$ \\
\hline & \begin{tabular}{|r|} 
OEL-OH \\
\end{tabular} & 二 & - & $=$ & $=$ & Joldures & $\frac{\varepsilon \varepsilon L-7 d S}{7 c 1-9}$ \\
\hline & $0 \mathrm{OELOH}$ & $=$ & $=$ & $=$ & - & 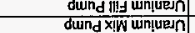 & $\frac{Z E I-d}{\mid E I-d}$ \\
\hline \multirow[t]{2}{*}{ 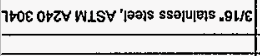 } & dVO IEM UNON & 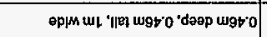 & 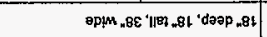 & - & $\rightarrow$ & xoqaiolo dund wnfueun & $\mathrm{OEL}-\mathrm{OH}$ \\
\hline & $\mathrm{OZT}-\mathrm{OH}$ & -- & & - & - & dwind litind & $z z L-d$ \\
\hline 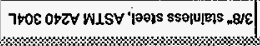 & $\forall \forall O \| E M Y S e M$ & 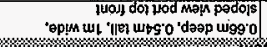 & 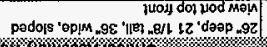 & $n_{d} b_{b}$ & - & xoganolg dund $\mathrm{nd}_{\mathrm{d}}$ & $\mathrm{OZLOH}$ \\
\hline 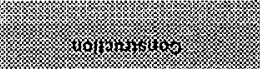 & 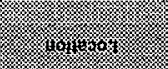 & 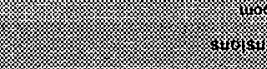 & (xime & Hing & 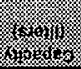 & 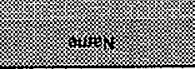 & 4 \\
\hline
\end{tabular}




\section{DISTRIBUTION SHEET}

\begin{tabular}{|c|c|c|c|c|c|}
\hline \multirow{2}{*}{$\begin{array}{l}\text { To } \\
\text { Distribution }\end{array}$} & \multirow{2}{*}{\multicolumn{3}{|c|}{$\begin{array}{l}\text { From } \\
\text { C. H. Brevick }\end{array}$}} & \multicolumn{2}{|l|}{ Page 1 of 2} \\
\hline & & & & \multicolumn{2}{|c|}{ Date July 7, 1997} \\
\hline \multirow{2}{*}{\multicolumn{4}{|c|}{$\begin{array}{l}\text { Project Title/Work Order } \\
\text { Engineering Study for Closure of } 209 \mathrm{E} \text { Facility/E61945 }\end{array}$}} & \multicolumn{2}{|c|}{ EDT No. 641029} \\
\hline & & & & \multicolumn{2}{|l|}{ ECN No. } \\
\hline Name & MSIN & $\begin{array}{l}\text { Text } \\
\text { With All } \\
\text { Attach. }\end{array}$ & Text Only & $\begin{array}{l}\text { Attach./ } \\
\text { Appendix } \\
\text { Only }\end{array}$ & $\begin{array}{l}\text { EDT/ECN } \\
\text { Only }\end{array}$ \\
\hline
\end{tabular}

Babcock \& Wilcox Hanford Company
R. W. Bailey
J. P. Hayfield
S6-15
S6-15
W. A. Peiffer
S6-15
L. D. Stefanski
S6-15

$$
\begin{aligned}
& X \\
& X \\
& X \\
& X
\end{aligned}
$$

Department of Energy Richland Operations

C. A. Ashley
J. D. Banks
M. L. Ramsay

Fluor Daniel Northwest

E. R. Amante

C. H. Brevick

S. D. Consort

L. A. Gaddis

E. D. Johnson

W. H. Hays

D. A. Lauha 7 a

D. A. Moody

E. A. Vickery

Engr. Publications

Link Technologies. Incorporated

E. N. Dodd III

Lockheed Martin Hanford Corporation

R. A. Dodd

L. R. Dunbar

M. S. Harrington

M. D. Long

P. C. Miller

R. P. Raven

W. E. Ross

J. W. Wicks

Lockheed Martin Services, Inc. Document Control $\begin{array}{ll}57-54 & X \\ 57-54 & X \\ 57-54 & X\end{array}$

E6-08 $X$

E6-08 $\quad X(5)$

E6-07 $X$

H5-57 $\quad X$

E6-08 $\quad X$

E6-08 $X$

E6-08 $X$

H5-57 $\quad X$

$\begin{array}{ll}E 6-08 & X \\ E 6-63 & X\end{array}$

S4-66 $X$

S5-07 $X$

H7-07 $\quad X$

R2-88 $X(10)$

R2-88 $X$

R1-5I $X$

R1-51 $X$

S5-07 $X$

R2-50 $X$

$R 1-29 \quad X$ 
HNF-SD-WM-ES-411, Rev. 0

Distribution Sheet

Page 2 of 2

Maintenance Concepts

C. E. Golden

R2-88

$\mathrm{R} 2-88$

B. Q. Peery

D. M. Stenkamp

R2-88

M. M. Jennings

$\mathrm{R} 2-88$

$x$

$x$

$x$

Waste Management Federal Services

P. A. Gagnon

S6-31

M. E. Lakes

S6-31

F. C. Schmidt

S6-30

M. L. Windsor

S6-30

$x$
$X$
$X$
$X$ 